\title{
Heusler Compounds and Spintronics
}

\author{
Chris J. Palmstrøm \\ University of California, Santa Barbara
}

\begin{abstract}
Heusler compounds are a large group of intermetallic compounds with over 1000 members with similar crystal structures having a vast array of tunable properties. These properties depend on the number of valence electrons per formula unit allowing tuning of properties through composition and alloying. The Heusler lattice parameters span many metal oxides and semiconductors and their crystal structures are closely related. For spintronic applications, the magnetic and half-metallic properties, in particular, are of great interest. In this chapter the electronic and magnetic properties of Heusler compounds are discussed as well as the importance of composition and defect control on tailoring their properties. Examples of applications include the great success of Heusler magnetic tunnel junction in metallic spintronic devices. The potential of going beyond metallic spintronics to the integration of Heusler compounds with III-V semiconductors for semiconductor spintronics device physics and technology, the tuning of magnetic properties, and the fabrication of Heusler compound heterostructures and superlattices are also discussed
\end{abstract}

Key words:

Heusler, full-Heusler, half-Heusler, spintronics, semiconductors, half-metals, magnetism, spin polarization, Schottky barrier, $\mathrm{Co}_{2} \mathrm{MnSi}, \mathrm{Co}_{2} \mathrm{FeSi}, \mathrm{Fe}_{3} \mathrm{Ga}$, $\mathrm{GaAs}$, NiMnSb, spin transfer torque, magnetic random access memory, MRAM, STT-RAM, MgO, GaAs, tunneling anisotropic magnetoresistance (TAMR), spin valve, magnetic tunnel junction, MTJ, spin polarization, valence electron count (VEC), Slater-Pauling, Spin-LED, thermodynamic stability, lattice mismatch, spin-FET, perpendicular magnetization, non-local voltage, molecular beam epitaxy, MBE, sputtering, density of states, point defects, stoichiometry, reflection high energy electron diffraction, RHEED, magnetic moment 


\section{Introduction:}

In this chapter the many properties of Heusler compounds will first be introduced, then their successful application in metallic spintronic devices (magnetic tunnel junctions) will be described, followed by a discussion on their applications beyond metallic spintronics and their integration with the well established III-V compound semiconductors and devices. Emphasis will be made on developing a detailed understanding of Heusler properties and how to integrate them with other materials, such as III-V compound semiconductors.

Heusler compounds are a large group of intermetallic compounds (Fig. 1) with $\sim 1500$ members that have a wide range of material properties[1] and can be thought of as the metallic equivalents to the Perovskite complex metal oxides. With such a large number of combinations of elements, many compounds have only recently been predicted theoretically to be thermodynamically stable and have yet to be experimentally verified[2,3]. In general, the Heusler compounds have face centered cubic crystal structures, which are either the full-Heusler or the half-Heusler structures (Fig. 2) with lattice parameters ranging from $\sim 5.6 \AA$ to $7.2 \AA$. The full-Heusler compound structures (Fig. 2) can be viewed as ordered B2 or stuffed $\mathrm{NaCl}$ crystal structures of $\mathrm{YZ}$ with every tetrahedral site occupied by $\mathrm{X}$. The half-Heusler compounds can be thought of as having only half of the $\mathrm{NaCl}$ tetrahedral sites filled by $\mathrm{X}$ or as an $\mathrm{XZ}$ zincblende lattice with the octahedral sites filled with Y. The half-Heusler compound CoTiSb, which is a semiconductor, may be thought of as a zincblende $\mathrm{sp}^{3}$ bonded $\mathrm{CoSb}^{4-}$ lattice with $\mathrm{Ti}^{4+}$ in the octahedral sites. The Heusler compound electronic properties are predicted to depend on the number of valence electrons per formula unit (VEC) [1,4-9]. Half-Heuslers with 17 or 19 VEC are predicted to be ferromagnetic (CoTiSn, CoVSb,..)[10], with 22 VEC half-metallic $(\mathrm{NiMnSb}, .).[6,11]$, and 8 or 18 VEC semiconductors (CoTiSb, NiTiSn,..) [1,7,8,12]. For the 18, 19 and 22 VEC half-Heusler compounds, covalent hybridization between the lower-energy dstates of the high valence transition metal $(\mathrm{X})$ and the higher-energy $\mathrm{d}$ states of the lower valence transition metal atom $(\mathrm{Y})$, leads to the formation of bonding and anti-bonding bands with a gap $\left(\mathrm{X} \mathrm{d} \mathrm{d}^{\mathrm{n}}, \mathrm{n}=0,1,4\right.$ for 18,19 and $\left.22 \mathrm{VEC}, \mathrm{Y} \mathrm{d}^{10}\right)$. The sp-elements $(\mathrm{Z})$, give rise to one $\mathrm{s}$ and three $\mathrm{p}$ bands below the center of the d-bands and can accommodate a total of 8 electrons $\left(\mathrm{s}^{2} \mathrm{p}^{6}\right)[4,6]$.

Full-Heuslers with 24 VEC are predicted to be semiconductors[5,13,14] and 26-29 can be superconductors $\left(\mathrm{Pd}_{2} \mathrm{HfAl}, ..\right)$ [15-19]. Some semiconducting Heuslers have also been predicted to be piezoelectric[20]. The dependence on VEC suggests that their properties can be tuned through alloying with elements to increase or decrease the number of electrons so as to adjust the position of the Fermi level. However, their properties and potential applications go far beyond what band gap engineering has enabled for conventional compound semiconductors [1].

For Spintronic application, the magnetic Heusler compounds and half-metals (Fig. 3), in particular, are of must interest. A number of review articles and books have been devoted to the magnetic and half-metal Heusler compounds[21-23]. The free carrier spin polarization at the Fermi level is an important property and is given by:

$$
P(E f)=D \uparrow(E f)-D \downarrow(E f) D \uparrow E f+D \downarrow(E f)
$$

where $D \uparrow$ and $D \downarrow$ are the spin-up and spin-down carrier density of states at the Fermi level. In half-metals the Fermi level is in the band gap for one spin (semiconducting) and in the band for 
the other (metallic). Hence the material will be 100\% spin polarized at the Fermi level (Fig. 3). This makes half-metals ideal for spintronic applications, as will be discussed below.

Figure 4 shows the calculated density of states for a number of half-Heusler compounds with 17, 18 and 19 VEC[24]. By increasing the electron count from 17 to 19 the Fermi level moves from the valence band to the conduction band and the properties change from magnetic to non-magnetic and back to magnetic. The magnetization depends on the number of magnetic moments per formula unit. For 3d metals Slater-Pauling behavior indicates that the magnetic moment per atom can be estimated from the average number of electrons per atom[25,26]. The total number of valence electrons per formula unit, $Z_{t}$ is given by

$$
Z_{\mathrm{t}}=\mathrm{N}_{\mathrm{v}} \uparrow+\mathrm{N}_{\mathrm{v}} \downarrow
$$

where $N_{v} \uparrow$ and $N_{v} \downarrow$ are the number of spin-up and spin-down electrons, respectively. The magnetic moment is the difference in the number of spin-up and spin-down electrons:

$$
\mathrm{M}_{\mathrm{t}}=\mathrm{N}_{\mathrm{v}} \uparrow-\mathrm{N}_{\mathrm{v}} \downarrow
$$

Half-Heuslers with 9 minority bands filled with 2 electrons in each band gives the "rule of 18 "[6]

$$
\mathrm{M}_{\mathrm{t}}=\mathrm{Z}_{\mathrm{t}}-18
$$

Figure 5 shows a plot of magnetic moment versus $Z_{t}$ for a number of half-Heusler compounds. For the full-Heusler compounds, the "rule of 24" applies[5] (Fig. 6). For the inverse Heusler compounds, in which the $\mathrm{X}$ and $\mathrm{Y}$ atoms are switched[1], the rules of 18, 24 and 28 apply[27] (see Fig. 7).

For a half-metal the minority spin bands are completely filled and the additional electrons must be in the majority band. This means that for a half-metal the magnetic moment per formula unit must be an integer number of Bohr magnetons, $\mu_{\mathrm{B}}$. The solid points in Figs. 5-7 indicate predicted half metallic compounds. The Slater-Pauling behavior of Heusler compounds allows one to make a direct comparison to conventional magnetic materials. Figure 8 shows the SlaterPauling curve for conventional magnetic alloys together with a number of full-Heusler compounds[1] and Fig. 9 a plot of Curie temperature versus VEC. An extensive review of halfmetal Heusler compounds can be found in reference [21].

A number of half-Heusler[6,11,28], full-Heusler[29-35] and inverse full-Heusler compounds are predicted to be half-metals[1,22,36,37]. Figure 10 shows the calculated band structure for the half-metal full-Heusler $\mathrm{Co}_{2} \mathrm{MnSi}[38]$. As can be seen in this figure, there are no minority spin states at the Fermi level. The majority spin band is metallic and the Fermi level is predicted to be in the middle of the minority spin band gap. However, defects can have a dramatic influence on the half-metallic properties. For the half-metallic half-Heusler compound $\mathrm{NiMnSb}$ Orgassa et al.[39] predicted that disorder (interchange of $\mathrm{Ni}$ and $\mathrm{Mn}$ or vacancies) would result in minority states at the Fermi level, dramatically decreasing the spin polarization. Similarly for $\mathrm{Co}_{2} \mathrm{MnSi}$ and $\mathrm{Co}_{2} \mathrm{MnGe}$, Picozzi et al.[40,41] found that the $\mathrm{Mn}$ antisite had the lowest formation energy, but still resulted in half-metallicity. On the other hand, Co antisites, which have slightly higher formation energies, have a dramatic effect on the half-metallicity. $\mathrm{Mn}-\mathrm{Si}$ and Mn-Co swaps have higher formation energy and keep the half-metallicity. Similar results were obtained by Özdogan and Galanakis[42] for $\mathrm{Co}_{2} \mathrm{MnSi}$ and $\mathrm{Co}_{2} \mathrm{MnAl}$. Mn-Si 
disorder retains the half-metallicity, but changes the crystal structure from the L $2{ }_{1}$ ordered Heusler structure to the disordered B2 crystal structure (Fig. 2). In order to enhance L $2{ }_{1}$ ordering, Heusler films are often annealed following deposition.

The surface properties of Heusler films may be very different from their bulk properties and will depend on the atomic termination[36,43-46]. Using argon ion sputter cleaning of the surface of $600^{\circ} \mathrm{C}$ annealed room temperature magnetron sputter deposited $\mathrm{Co}_{2} \mathrm{MnSi}(001)$ films on $\mathrm{MgO}(001)$ and spin-resolved photoemission spectroscopy, Wüstenberg et al.[47] reported that for Mn-poor films $\left(\mathrm{Co}_{2} \mathrm{Mn}_{0.69} \mathrm{Si}_{1.01}\right)$ the spin polarization at the Fermi energy $\left(\mathrm{E}_{\mathrm{f}}\right)$ was negative due to the presence of $\mathrm{Co}_{\mathrm{Mn}}$ antisites and minority surface state contributions. In Mn-rich compounds, $\mathrm{Co}_{2} \mathrm{Mn}_{1.19} \mathrm{~S}_{\mathrm{i} 0.88}$, they reported that the suppression of $\mathrm{Co}_{\mathrm{Mn}}$ antisites leads to a positive spin polarization at $\mathrm{E}_{\mathrm{f}}$, and the influence of minority surface states on the photoelectron spin polarization is reduced. An in-situ spin-resolved UV photoemission study of radio frequency (RF) sputter deposited at room temperature and in-situ $550^{\circ} \mathrm{C}$ annealed $\mathrm{Co}_{2} \mathrm{MnSi} / \mathrm{MgO}(001)$ films yielded a $93 \%$ surface spin polarization at room temperature[48]. A more detailed in-situ spin polarized photoemission study of molecular beam epitaxy (MBE) grown and $\sim 750^{\circ} \mathrm{C}$ annealed $\mathrm{Co}_{2} \mathrm{Mn}_{1+\delta} \mathrm{Si} / \mathrm{MgO}(001)$ films by Andrieu et al.[46] showed that $\sim 100 \%$ spin polarization occurred below the Fermi level for stoichiometric films $(\delta=0)$ (Figs. 11 and 12). This indicates that the Fermi level is above the optimum position in the gap for the minority states in the bulk as well as at the surface (Fig. 11). They demonstrated that the Fermi level could be tuned to enhance the spin polarization at the Fermi level (Fig. 11) by increasing the Mn concentration ( $\delta>0$ ) (VEC tuning) or by one monolayer deposition of $\mathrm{Mn}, \mathrm{MnSi}$, or $\mathrm{MgO}$ (Fig. 12). These results demonstrate that bulk composition and surface termination have a strong influence on the spin polarization.

In addition to the surface, the spin polarization at an interface is also predicted to depend on the atomic structure of the interface[41,49]

\section{Heusler Device Structures:}

\section{Metal Spintronics: Magnetic Tunnel Junction and Magnetic Spin Valve}

The concept of a spintronic device is to utilize the spin rather than the charge of the electron. The magnetic tunnel junction (MTJ) and magnetic spin valve (SV) are examples of metallic spintronic devices. These consist of ferromagnet/insulator tunnel barrier/ferromagnet (MTJ) and ferromagnet/normal metal/ferromagnet (SV) structures and are shown in Fig. 13. For the MTJ, transport of electrons from one ferromagnetic contact to the other occurs via tunneling through the insulating barrier. The resistance of spin valves and MTJ devices depends on the relative alignment for the two ferromagnetic layers. The insert in Fig. 13 shows the schematic of the density of states for two half-metallic ferromagnet contacts for the parallel and antiparallel magnetic states. When the two ferromagnetic layers are aligned parallel, there are states for the spin down electrons at the Fermi level to go from the one ferromagnetic layer into the second. When they are antiparallel, the states at the Fermi level have opposite spin for the two ferromagnets; although there are states available in the normal metal, there are no states for the spin down electrons to enter the second ferromagnet without flipping their spin. Hence, in this configuration, the spin valve will be in its high resistance state. This is schematically shown in Fig. 13. In the case of the MTJ, the transport would be by tunneling through the insulating tunnel 
barrier. Following the approach of Julliere [50], the tunneling magnetoresistance (TMR) is given by:

$$
T M R / V=O=G P-G A P G A P=R A P-R P R P=2 P^{\prime} 1 P^{\prime} 21-P^{\prime} 1 P^{\prime} 2
$$

where $G_{P}, G_{A P}, R_{P}$, and $R_{A P}$ are the tunneling conductance $(d I / d V)$ and tunneling resistance for the parallel and antiparallel configurations. $\mathrm{P}_{1}^{\prime}$ and $\mathrm{P}_{2}^{\prime}$ are the tunneling spin polarization for the two ferromagnetic contacts, respectively. Note that $\mathrm{P}^{\prime}{ }_{1}$ and $\mathrm{P}_{2}{ }_{2}$ are not the carrier spin polarizations $\mathrm{P}_{1}$ and $\mathrm{P}_{2}$ in the ferromagnetic contacts. $\mathrm{P}_{1}^{\prime}$ and $\mathrm{P}^{\prime}{ }_{2}$ also depend on the electron effective mass, interface bonding and scattering and the spin dependent attenuation in the tunnel barrier. For perfect half-metal contacts $\mathrm{P}_{1}\left(\mathrm{E}_{\mathrm{f}}\right)$ and $\mathrm{P}_{2}\left(\mathrm{E}_{\mathrm{f}}\right)$ would be 1 and, assuming no spin flip scattering, this would result in $\mathrm{P}_{1}^{\prime}$ and $\mathrm{P}_{2}^{\prime}$ also being 1 and the TMR would be infinity.

From the discussion above, it is clear that the measured spin polarization depends upon the measurement technique used and care must be taken when comparing different spin polarization measurements. In general, the spin polarization is given by:

$P(E f) n=v f \uparrow n D(E f) \uparrow-v f \downarrow n D(E f) \downarrow v f \uparrow n D(E f) \uparrow+v f \downarrow n D(E f) \downarrow$

where $v f \uparrow n$ and $v f \downarrow n$ are the Fermi velocities for spin up and spin down electrons, respectively. The density of states is weighted by the Fermi velocity raised to the power of $n$, which is 0 for electrons ejected in a spin-polarized photoemission experiment (as in Figs. 11 and 12), 1 for ballistic transport (i.e. tunneling, as in a TMR measurement) and 2 for diffusive transport or tunneling at low bias[51].

In the MTJ, Butler et al. [52] and Mathon and Umersky[53] predicted that the tunneling conductance depends strongly on the band structure of the barrier as this would have a dramatic effect on how the states in the ferromagnetic contacts decay in the barrier. In the case of $\mathrm{Fe} / \mathrm{MgO} / \mathrm{Fe}$ they predicted a very strong spin filtering effect of the barrier. In $3 \mathrm{~d}$ ferromagnetic metals the Bloch states with $\Delta_{1}$ symmetry (spd hybridized states) tend to have large majority spin polarization at the Fermi level. Bloch states with $\Delta_{5}$ (pd states) and $\Delta_{2}$ symmetry (d states) have smaller or minority (opposite) spin polarization at the Fermi level. In $\mathrm{MgO}$ the attenuation is much larger for states with $\Delta_{5}$ and $\Delta_{2}$ than $\Delta_{1}$ symmetries (see Fig. 14), resulting in preferential tunneling of majority spins. This spin filtering effect can substantially increase the measured TMR. The use of epitaxial $\mathrm{MgO}(001)$ tunnel barriers rather than $\mathrm{AlO}_{\mathrm{x}}$ amorphous barriers resulted in a dramatic increase in TMR[54-56].

$\mathrm{MgO}(001)$ tunnel barriers have also resulted in a dramatic increase over $\mathrm{AlO}_{\mathrm{x}}$ tunnel barriers for Heusler compound based MTJs. Figure 15 shows the improvement in TMR with change in tunnel barrier material, time and variations in Heusler composition (data obtained from references [57-67]). These are all structures made using sputter deposition and post deposition annealing rather than MBE. The TMR is substantially higher at low temperature with the record TMR ratios of $2610 \%$ at $4.2 \mathrm{~K}$ and $429 \%$ at $290 \mathrm{~K}$ for a $\mathrm{Co}_{2} \mathrm{Mn}_{1.24} \mathrm{Fe}_{0.16} \mathrm{Si}_{0.84}$ composition film[67]. From the figure, the films with excess Mn, Fe and deficient Si have the highest TMR. From the arguments above, the excess $\mathrm{Mn}$ should minimize the number of $\mathrm{Co}_{\mathrm{Mn}}$ antisite defects that would reduce the spin polarization. An alternate explanation would be that the Fermi level is being tuned into the middle of the gap for minority spin density of states by VEC tuning[46]. Liu 
et al. [67] have done one of the most extensive studies on the influence of composition on the TMR and conclude that both mechanisms are responsible for their record TMR results. This conclusion can be seen from Fig. 16, which shows the TMR ratio data from reference [67] plotted versus the Y-element concentration $(\mathrm{Mn}+\mathrm{Fe})$ (Fig. 16(a) and (b)) and VEC (Fig. 16(c) and $(\mathrm{d}))$. It is clear that for $\mathrm{Co}_{2} \mathrm{Mn}_{0.73+\delta} \mathrm{Si}_{0.84}$ the TMR is strongly dependent upon VEC. The decrease in the TMR ratio with increasing VEC for $\mathrm{Co}_{2} \mathrm{Mn}_{0.73+\delta} \mathrm{Si}_{0.84}$ is consistent with the recent spin polarization measurements of $\mathrm{Co}_{2} \mathrm{Mn}_{1+\delta} \mathrm{Si}$ of Andrieu et al.[46], which suggest that the spin polarization at the Fermi level decreases as the Fermi level increases in energy (Fig. 11), i.e. as VEC increases. However as discussed above, it is difficult to make a quantitative comparison between the spin polarization measured by photoemission, which measures the density of states, and the MTJ, which measures the spin dependent transport through a tunnel barrier. Furthermore, the details of the surfaces and interface are important.

Figure 17 shows a high angle annular dark field scanning transmission electron microscopy (HAADF-STEM) image of an MTJ with $\mathrm{Co}_{2} \mathrm{Mn}_{1.24} \mathrm{Fe}_{0.16} \mathrm{Si}_{0.84}$ ferromagnetic layers. This corresponds to the MTJ with the highest TMR ratio shown in Fig. 15. Note that although the interface appears abrupt, it is not atomically smooth. There are clear atomic variations in the $\mathrm{MgO}$ thickness. Furthermore, Figs. 17(b) and (c) show that the $\mathrm{Co}_{2} \mathrm{Mn}_{1.24} \mathrm{Fe}_{0.16} \mathrm{Si}_{0.84}$ is mixed phase with both the disordered B2 (region b) and ordered L2 1 (region c) crystal structures, respectively. The fact that these MTJ have shown the highest TMR ratios to date suggests that the $\mathrm{B} 2$ disorder does not have a strong influence on the spin polarization, which is in agreement with theory[40-42].

\section{Beyond MTJs and Metal Spintronics: Integration with III-V Semiconductor Technology}

Metallic MTJ and GMR devices have been highly successful in applications such as hard discs read heads and magnetic random access memory (MRAM) devices. Integration with semiconductors has the potential for developing devices with new function. The spin-field effect transistor (spin-FET)[68] (Fig. 18), spin-LEDs and spin-laser[69-71], as well as spin logic devices[72] are examples. In the spin-FET, ferromagnetic contacts are used for spin injection and detection into a two-dimensional electron gas (2DEG) in a semiconductor with large spin-orbit coupling. The spin precession due to spin-orbit coupling is controlled by the applied voltage on the Schottky gate, which produces a perpendicular electric field that results in an effective magnetic field (Rashba) that makes the spins precess in the channel [68]. Spin-induced gain anisotropy on output polarization and reduced threshold current have been demonstrated for quantum dot vertical cavity surface emitting lasers (VCSELs) using ferromagnetic spin injecting contacts[69,70]. The high spin polarization of Heusler compounds would make these ideal potential candidates for spin contacts to semiconductors. However, spin polarization is not the only significant factor; lattice matching, thermodynamic stability, interfacial bonding and electronic properties are all important[73]. $\mathrm{MgO}$ is remarkably stable against reactions with transition metals, whereas III-V semiconductors are much more reactive. Hence the approach of high temperature annealing to achieve ordering of the Heusler films for MTJs is unlikely to be possible for growth on III-V semiconductors.

\section{Lattice Matching}


As shown in Fig. 2, the Heusler compounds have fcc crystal structures $\left(\mathrm{L} 2{ }_{1}, \mathrm{C}_{\mathrm{b}}\right.$, and $\mathrm{DO}_{3}$ ) that are closely related to the III-V semiconductor zincblende structure. Fig. 19 shows a plot of lattice parameter versus element for a number of Heusler compounds. The plot indicates the lattice parameters of a number of common III-V semiconductors. As can be seen from this figure, $\mathrm{Co}_{2} \mathrm{MnSi}$ and $\mathrm{Co}_{2} \mathrm{FeSi}$ are closely lattice matched to GaAs, suggesting that these may be ideal spin contacts to GaAs. As discussed above, these are expected to have high spin polarization.

\section{Thermodynamic stability}

The Heusler compound/III-V semiconductor heterostructure is unlikely to be thermodynamically stable as this would typically involve 5 elements with the potential for the formation of a very large number of different compounds through multiple combinations of the elements, such as between $\mathrm{Co}, \mathrm{Mn}, \mathrm{Si}, \mathrm{Ga}$ and $\mathrm{As}$ for a $\mathrm{Co}_{2} \mathrm{MnSi} / \mathrm{GaAs}$ heterostructure. Although a number of metal-III-V ternary phase diagram predictions and measurements have been made[74,75], quaternary and penternary phase diagrams have not been made. There have been numerous studies of metal/III-V semiconductor contact reactions[76-79]. In general, most elements will react with GaAs and other III-V semiconductors. Si and Ge are two of the elements that are predicted to be thermodynamically stable in contact with GaAs[74]. Although the individual elements are not thermodynamically stable in contact with III-V semiconductors, metallic compounds of the elements can be [78,79]. Although the relative stability of each Heusler compound and III-V semiconductor is not known, one can make some intuitive predictions on which systems may be more stable than others using knowledge of the reaction kinetics of the individual elements with the III-V semiconductor. In the case of transition elements and GaAs, the interfacial reaction kinetics are fastest for $\mathrm{Ni}[80]$, then $\mathrm{Mn}[81]$, $\mathrm{Co}$ [8284], and the slowest is $\mathrm{Fe}[85,86]$. Hence, one may expect Ni based Heusler compounds to be more likely to have higher interfacial reaction kinetics than Fe based ones.

From Fe/GaAs thin film reaction studies Schultz et al. predicted the Fe-Ga-As phase diagram shown in Fig. 20[86] which indicates that Fe is not thermodynamically stable in contact with $\mathrm{GaAs}$, but $\mathrm{Fe}-\mathrm{Ga}$ and $\mathrm{Fe}-\mathrm{As}$ phases are and, in particular, $\mathrm{Fe}_{3} \mathrm{Ga}$. In this study they found that the $\mathrm{Fe}_{3} \mathrm{Ga}$ grew in the metastable $\mathrm{DO}_{3}$ structure, possibly due to epitaxial stabilization by the GaAs substrate. As discussed above, this $\mathrm{DO}_{3}$ structure is essentially the Heusler L2 $2_{1}$ crystal structure with elements $\mathrm{X}$ and $\mathrm{Y}$ being the same (i.e. $\mathrm{Fe}_{2} \mathrm{FeGa}$ ) (Fig. 2).

\section{Heusler Compound Growth on III-V Semiconductors}

The prediction of half-metallicity for the half-Heusler compounds $\mathrm{NiMnSb}$ and $\mathrm{PtMnSb}[11]$ and for the full-Heusler compounds $\mathrm{Co}_{2} \mathrm{MnGe}$ and $\mathrm{Co}_{2} \mathrm{MnSi}[30]$ precipitated strong interest in pursuing experimental verification of half-metallicity by epitaxial Heusler compound growth. Unlike metallic MTJs, where sputtering has been the technique of choice, most Heusler compound growth on semiconductors has generally been by molecular beam epitaxy (MBE) using individual elemental sources. Initial Heusler growth studies on III-V semiconductors were aimed at studies of the film properties, particularly the magnetic and electronic properties, rather than the Heusler compound/III-V heterostructure properties. A number of Heusler compounds were successfully grown on III-V semiconductors. These include: $\mathrm{Fe}_{3}(\mathrm{Al}, \mathrm{Si})$ [87-89], $\mathrm{Ni}_{2} \mathrm{MnGa}$, $\mathrm{Ni}_{2} \mathrm{MnAl}, \mathrm{Ni}_{2} \mathrm{MnIn}$ and $\mathrm{Ni}_{2} \mathrm{MnGe}[90-96], \mathrm{Co}_{2} \mathrm{MnGe}$ [97-100], $\mathrm{Co}_{2} \mathrm{MnSi}[101,102], \mathrm{Co}_{2} \mathrm{FeSi}[103-$ 
106], $\mathrm{Co}_{2} \mathrm{MnGa}[107], \mathrm{Co}_{2} \mathrm{NiGa}[108]$ and the half-Heusler NiMnSb[109-114]. These studies clearly demonstrated high crystal quality epitaxial growth. Point Contact Andreev Reflection (PCAR) measurements of the spin polarization of $\mathrm{Co}_{2} \mathrm{FeSi}$ films suggest a polarization $\leq 60 \%[106,115]$, similar to other Heusler compounds[116,117] including NiMnSb and $\mathrm{Co}_{2} \mathrm{MnSi}[118,119]$. PCAR measurements were performed on air exposed films. In-situ spinpolarized photoemission measurements of $\mathrm{Co}_{2} \mathrm{MnSi}$ epitaxial films[46,48], discussed above, yielded much higher spin polarization ( $>90 \%)$. This suggests that either the in-situ prepared films were of higher crystal quality or air exposure significantly alters the film properties. An alternate explanation for the large difference in transport spin polarization (PCAR) and the density of states spin polarizations is a difference in the Fermi velocity for the two spins, as discussed above (equation (5)). Discussions above also indicated that Y-Z atomic disorder should, in general, not have a large influence on the spin polarization. However, this is not always the case. For instance, $\mathrm{Ni}_{2} \mathrm{MnAl}$ is ferromagnetic in the ordered $\mathrm{L} 2{ }_{1}$ structure and is antiferromagnetic in the B2 [120,121]. Dong et al.[10] found that by controlling the substrate temperature during MBE growth, antiferromagnetic or ferromagnetic $\mathrm{Ni}_{2} \mathrm{MnAl}$ films could be grown. Antiferromagnetic epitaxial $\mathrm{Ni}_{2} \mathrm{MnAl}$ in the $\mathrm{B} 2$ structure would grow at $180^{\circ} \mathrm{C}$ while growing at $400^{\circ} \mathrm{C}$ resulted in $\mathrm{L} 2{ }_{1}$ ordering and being ferromagnetic.

\section{Growth of Heusler Compound Contacts for Semiconductor Spintronics}

In the preceding sections the focus has been on the properties of the Heusler compounds; however, when integrating Heusler compounds with III-V semiconductors, the interfacial properties are critical. The large impedance mismatch between a metallic Heusler compound and a semiconductor requires the transport to be ballistic (i.e. tunneling) rather than diffusive in order to achieve significant spin polarization[122]. Fortunately, Fermi level pinning results in metal contacts to GaAs being Schottky barriers rather than ohmic contacts. Hence, in order to ensure sufficient tunneling through the Schottky barrier, the semiconductor immediately beneath the contact must be heavily doped so as to have a shallow depletion width and narrow barrier[123,124]. Furthermore, the details of the narrow degenerate doping region in the vicinity of the ferromagnet/semiconductor interface plays an essential role in the spin injection and detection[125]. In addition, interfacial reactions can have a dramatic effect on the spin transport[126-128]. These results indicate that the properties of Heusler/III-V semiconductors are likely to be very sensitive to both the details of the III-V semiconductor interfacial doping profile, the semiconductor surface preparation and the heteroepitaxial growth process itself.

The surface composition and reconstruction of III-V semiconductors depends on temperature and group-V overpressure. Figure 21 shows the surface phase diagram for $\operatorname{GaAs}(001)[129]$. During MBE growth, the typical surface reconstruction is the $(2 \times 4) / c(2 \times 8) \beta 2$ with $\sim 0.75$ monolayer As coverage[130]. Upon cooling under an As-flux, this surface adsorbs more As and converts to a $\mathrm{c}(4 \mathrm{x} 4)$ reconstruction with $\sim 1.75$ monolayer As coverage. Other reconstructions and terminations can be achieved through annealing and controlling of the Ga and As fluxes[130]. It is important to remember that although GaAs is a cubic crystal, the (001) surface has two fold surface symmetry and therefore the [110] and [1-10] directions are not equivalent due to the $\mathrm{sp}^{3}$ bonding. This tends to result in interfacial induced uniaxial magnetic anisotropy of epitaxial magnetic films[131]. 
In the experimental results described below, a multi chamber ultra-high vacuum (UHV) growth and in-situ characterization system was used to grow, characterize and study the growth process of Heusler compound/GaAs heterostructures.

\section{Measurement of spin transport at Heusler/III-V semiconductor interfaces}

Spin-LED

Initial demonstration of spin injection from a ferromagnetic contact into III-V semiconductor heterostructures relied on the use of a spin-LED[123,124,132-134]. Figure 22 shows a schematic of a spin-LED structure. The ferromagnetic-/semiconductor Schottky barrier is reverse biased to inject electrons into the n-type semiconductor and the $p$-n junction is forward biased. The injected electrons recombine with holes from the p-type layer in the $p$-n junction depletion region, which in this case is an undoped region with a GaAs quantum well (QW). A magnetic field is applied perpendicular to the contact to ensure that the ferromagnetic contact magnetization is pulled out of plane so the injected electron spin orientation is perpendicular to the surface. Measurement of the left and right circularly polarized light gives, through the optical selection rules, the spin polarization of the electrons recombining with holes in the quantum well. The spin polarization should track with the out of plane magnetization of the ferromagnetic contact. Although this is a direct measure of the spin polarization in the semiconductor quantum well, it has several drawbacks. It can only be used for semiconductors with a direct band gap and it is essentially a back-to-back diode, which makes it difficult to determine the potential profile within the device. The first demonstration of spin injection from a Heusler compound into GaAs was reported by Dong et al. for $\mathrm{Co}_{2} \mathrm{MnGe}$ [135] and later for $\mathrm{Co}_{2} \mathrm{FeSi}$ by Ramsteiner et al.[105] using spin-LEDs.

\section{Non-local lateral Spin Valve measurements}

Measurement of spin transport in lateral ferromagnet/semiconductor devices offers many advantages over spin-LEDs as spin injection, transport and detection can all be measured in a single device[136,137]. Figure 23 shows a schematic of the contacts and the non-local measurement. The doping profile in the GaAs is similar to that used in the spin-LED. However, the $\mathrm{n}^{+}$contact layer is etched away between the ferromagnetic contacts to ensure that the lateral transport is in the n-type GaAs channel. Spin-polarized electrons are injected into the GaAs channel at contact $C$ and flow towards contact $A$, while the voltage, $V_{D, E}$, is measured between contacts D and E. Although current flows between contacts $\mathrm{C}$ and $\mathrm{A}$, non-equilibrium spin polarization in the GaAs can diffuse in either direction from the source. The spin polarization results in an electrochemical potential difference, $\Delta \mu$, for the two spin states in the channel, leading to a change in $\mathrm{V}_{\mathrm{D} \text {.E }}$ when the magnetization of contact $\mathrm{D}$ is switched antiparallel to contact $\mathrm{C}$, to the parallel configuration. The GaAs spin polarization at contact $\mathrm{E}$ is always zero as it is many spin diffusion lengths away. The spin-valve measurement is carried out by sweeping the magnetic field along the magnetic easy axis of the contacts and looking for a change in voltage over the narrow field range in which contacts $C$ and $D$ are antiparallel. The top curve in Fig. 23(d) is the raw signal and the bottom is the signal after the linear background has been subtracted. By setting the contacts $\mathrm{C}$ and $\mathrm{D}$ in parallel and antiparallel states and applying a small varying out of plane magnetic field, Hanle measurements can be made. Fig 23(e) shows 
the raw signal (top curve) and after background subtraction (bottom curve). By varying the current and bias of contact $\mathrm{C}$, the GaAs spin polarization can be measured as a function of forward (spin extraction) and reverse (spin injection) bias. Magnetoptic Kerr effect (MOKE) can also be used to measure the spin polarization directly in the Ga As channel[137-139]. Furthermore, by biasing of the detector the spin detection sensitivity can be increased significantly[137,139].

\section{$\mathrm{Fe}_{3} \mathrm{Ga} / \mathrm{GaAs}(001)$}

Thin film Fe/GaAs reaction studies by Schultz et al.[86] found that $\mathrm{Fe}_{3} \mathrm{Ga}$ with the $\mathrm{DO}_{3}$ related Heusler structure formed after $450^{\circ} \mathrm{C}$ annealing and that it should be thermodynamically stable in contact with GaAs as is shown in the phase diagram in Fig. 19. The mismatch between $\mathrm{Fe}_{3} \mathrm{Ga}$ in the $\mathrm{DO}_{3}$ structure $(5.812 \AA)$ and GaAs $(5.6533 \AA)$ is $\sim 3 \%$. Figure 24 shows x-ray diffraction scans for a $30 \mathrm{~nm}$ thick $\mathrm{Fe}_{3} \mathrm{Ga}$ film grown by codeposition at $325^{\circ} \mathrm{C}$ on $\mathrm{GaAs}(001)$. The film grows in the $\mathrm{DO}_{3}$ structure and the reciprocal lattice scan around the GaAs(224) peak indicates that the film is growing pseudomorphic on the $\operatorname{GaAs}(001)$ surface with an out of plane lattice parameter of $6.12 \AA$. The finite thickness fringes indicate a relatively smooth film and sharp interface.

When considering the growth of $\mathrm{Fe}_{3} \mathrm{Ga}$ on $\mathrm{GaAs}(001)$ one has to consider the atomic details of the growth process as shown in Fig. 25. The first question to be answered is to determine which GaAs surface reconstruction should be used. As-rich or Ga-rich reconstructions can promote specific interfacial phase formations. For example, an As-rich surface may promote the formation of Fe-As compounds, which can be antiferromagnetic. A second consideration is the stacking sequence in $\mathrm{Fe}_{3} \mathrm{Ga}$ itself, which in the [001] direction involves sequential layers of $\mathrm{Fe}+\mathrm{Ga}$ and $\mathrm{Fe}$. The $\mathrm{Fe}_{3} \mathrm{Ga}$ growth can then be initiated with a layer of $\mathrm{Fe}$ or $\mathrm{Fe}+\mathrm{Ga}$ followed by sequential atomic layers, by codeposition or a mixture of sequential layers and codeposition. To test the influence of the growth sequence, $\operatorname{GaAs}(001)$ heterostructures for lateral transport measurements as shown in Fig. 23 were grown and then different surface reconstructions were prepared immediately prior to $\mathrm{Fe}_{3} \mathrm{Ga}$ growth. The $\mathrm{Fe}_{3} \mathrm{Ga}$ initial growth sequence was also varied. Fig. 26 shows the procedures used to create different interfaces.

Figure 27 shows high angle annular dark field scanning transmission electron microscopy (HAADF-STEM) images of the $\mathrm{Fe}_{3} \mathrm{Ga} / \mathrm{GaAs}(001)$ As-rich and Ga-rich interface samples[140]. The interfaces appear atomically abrupt suggesting minimal interfacial reactions. However, the interfaces are clearly different between the [1-10] and [110] cross-sections as would be expected for the two fold surface symmetry. In addition, there is a clear difference in interface structure between the two samples. The [110] cross section of the Ga-rich interface sample shows additional atomic structures every three Ga-As dumbbells at the interface. This is interpreted as remanence of the Ga-rich $\mathrm{GaAs}(001) \mathrm{c}(8 \times 2)$ reconstruction[130].

Lateral transport spin valves were fabricated and measured for the differently prepared interfaces and the bias dependence of the spin polarization electrochemical potential difference (spin valve signal) is shown in Fig. 28. Clearly, the Ga initiated growth on GaAs(001) $(4 \times 6) / c(8 \times 2)$ Ga-rich surface interface sample (sample A) has the highest spin valve signal.

\section{$\mathrm{Co}_{2} \mathrm{Mn}_{1-\mathrm{x}} \mathrm{Fe}_{\mathrm{x}} \mathrm{Si} / \mathrm{GaAs}(001)$}


As discussed above, $\mathrm{Co}_{2} \mathrm{MnSi}$ is predicted and recently confirmed by in-situ spin polarized photoemission measurements to be $>90 \%$ spin polarized $[46,48]$. This together with the close lattice match to GaAs (0.06\% mismatch) and high Curie temperature $(985 \mathrm{~K})$, makes $\mathrm{Co}_{2} \mathrm{MnSi}$ very attractive as a potential spin contact to GaAs.

Using effusion cells for $\mathrm{Co}$ and $\mathrm{Mn}$ and a single crystal $\mathrm{Si}$ strip heater cell for $\mathrm{Si}$, $\mathrm{Co}_{2} \mathrm{MnSi}$ films were grown on $\mathrm{GaAs}(001) \mathrm{c}(4 \mathrm{x} 4)$ As-rich surfaces[141]. Streaky RHEED patterns were observed during growth as were RHEED intensity oscillations indicative of a layer-by-layer growth mode at a substrate temperature of $270^{\circ} \mathrm{C}$ (Fig. 29). The effusion cell fluxes were set using a beam flux monitor that had been calibrated using Rutherford backscattering measurements of calibration samples grown on Si substrates. In-situ scanning tunneling microscopy (STM) images showed step heights corresponding to 1 bilayer $(2.8 \AA)$ of $\mathrm{Co}_{2} \mathrm{MnSi}$ (Fig. 29(c)), consistent with the layer-by-layer growth inferred from the RHEED intensity oscillations Fig. 29(b)). The 2-fold surface symmetry of GaAs (001), combined with the thin film geometry, induces a strong in plane uniaxial magnetic anisotropy on the $\mathrm{Co}_{2} \mathrm{MnSi}_{\text {with }}$ an easy axis in the [110] direction (Fig. 29(d)).

The spin polarized photoemission measurements only measure the surface of the films and not the buried interface. For spin transport at the $\mathrm{Co}_{2} \mathrm{MnSi} / \mathrm{GaAs}$ interface, the spin polarization at the interface is critical. There are many theoretical studies predicting the interfacial spin polarization for a number of different Heusler/III-V semiconductor heterostructures. These include $\mathrm{Co}_{2} \mathrm{MnGe} / \mathrm{GaAs}[41,142,143], \quad \mathrm{Co}_{2} \mathrm{MnSi} / \mathrm{GaAs}[143-146]$, $\mathrm{Co}_{2} \mathrm{FeSi} / \mathrm{GaAs}[147], \quad \mathrm{Co}_{2} \mathrm{CrSi} / \mathrm{GaAs}[143], \quad \mathrm{Co}_{2} \mathrm{CrAl} / \mathrm{InP}[148], \quad \mathrm{NiMnSb} / \mathrm{GaAs}[149] \quad$ and $\mathrm{NiMnSb} / \mathrm{InP}$ [149]. In general, the predicted interfacial spin polarization is found to depend strongly on the interfacial atomic configuration and crystal orientation.

As in the case for $\mathrm{Fe}_{3} \mathrm{Ga} / \mathrm{GaAs}(001)$ discussed above, different growth sequences may result in different interfacial structures. Looking at the $\mathrm{Co}_{2} \mathrm{MnSi} 2_{2}$ Heusler structure (Fig. 2) it is clear that the [001] stacking sequence consists of alternate layers of $\mathrm{Mn}+\mathrm{Si}$ and $\mathrm{Co}$. By performing sequential atomic layer depositions and in-situ analysis using RHEED, STM and xray photoelectron spectroscopy (XPS) after each layer, a picture of the different interface formations can be inferred. Starting with $\mathrm{GaAs}(001) \mathrm{c}(4 \mathrm{x} 4)$ As-rich surfaces, two different growth sequences were investigated. The first involved initiating the growth with 1 monolayer (ML) of Mn-Si followed by 1ML Co and then $1 \mathrm{ML}$ Mn-Si in an atomic layer by atomic layer growth mode. The second used the same growth mode, but initiated the growth with 1ML Co. RHEED of a starting $\mathrm{c}(4 \mathrm{x} 4) \mathrm{GaAs}(001)$ surface as well as after the deposition of each monolayer in Co- and $\mathrm{MnSi}$-initiated samples of $\mathrm{Co}_{2} \mathrm{MnSi}$ is shown in Fig. 30. After the deposition of the first monolayer, there are no significant differences in RHEED, and after deposition of $15.4 \mathrm{~nm}$ of film, the RHEED patterns are nearly identical, showing a slight $2 \mathrm{x}$ periodicity in both the [110] and [110] directions. The corresponding XPS measurements (Fig. 31 ) of the Ga $3 d$ peak intensity attenuates slower than expected indicating that $\mathrm{Ga}$ is either riding on the film surface or the $\mathrm{Co}_{2} \mathrm{MnSi}$ is growing as islands and not completely covering the GaAs surface. From the in-situ STM images of the $\mathrm{Co}_{2} \mathrm{MnSi}$ it is evident that the $\mathrm{Co}_{2} \mathrm{MnSi}$ film after the first few ML is not growing as islands and the Ga must therefore be riding on the surface. The intensities of the $\mathrm{Ga} 3 \mathrm{~d}$, Co $2 \mathrm{p}$ and $\mathrm{Mn} 2 \mathrm{p}$ peaks as a function of monolayer deposition are shown in Fig. 31 for the two different growth sequences. Intensities are expected to increase upon deposition of the layer that contains the element, and are expected to attenuate after deposition of the next layer. The trend in the MnSi initiated sample is consistent with this description, but for Co initiations, the MnSi layer does not attenuate after the first layer, 
suggesting that the $\mathrm{Mn}$ is going beneath the Co. Regardless of the nucleation sequence, the $\mathrm{Co}_{2} \mathrm{MnSi} / \mathrm{GaAs}(001)$ interface is similar and Mn-rich (Fig. 32).

Electrical measurements are generally more sensitive than XPS. The room temperature current-voltage characteristics of $\mathrm{Co}_{2} \mathrm{MnSi} / \mathrm{GaAs}(001)$ contacts with $\mathrm{Co}, \mathrm{Mn}, \mathrm{Si}$, and $\mathrm{Mn}-\mathrm{Si}$ nucleation layers were compared and are shown in Fig. 33(a). The interpretation of these data is that the Co and Si initiated contacts have similar effective Schottky barrier heights and that it is higher for Mn-Si and highest for Mn. This increase in the effective Schottky barrier height could either be a result of a true Schottky barrier height increase or due to a decrease in the $n+$ type doping in the GaAs. Since Mn is a p-type dopant in GaAs and any Mn diffusion into the GaAs would result in compensation of the Si n-type doping. This would increase the Schottky barrier width, decreasing the tunneling probability, and therefore increase the effective Schottky barrier height. Fig. 33(b) shows a backside secondary ion mass spectrometry (SIMS) depth profile of the different elements for a structure with MBE-grown AlGaAs marker layers for sputter depth profile calibration[150]. The signal for $\mathrm{Mn}$ rises significantly before the $\mathrm{Co}$ and $\mathrm{Si}$ signals, indicating Mn diffusion into the GaAs. In addition, the profile is also consistent with a Mn rich interface, in agreement with the in-situ XPS studies. Ebina et al.[102] used conventional front side SIMS, which is more prone to ion beam mixing and knock-on resulting in decreased depth resolution, to detect $\mathrm{Mn}$ indiffusion from sputter deposited $\mathrm{Co}_{2} \mathrm{Mn}_{1.30} \mathrm{Si}_{0.84}$ films and concluded that this was the cause of no clear spin signal. They found that the insertion of a thin FeCo layer suppressed the Mn indiffusion and observed high spin injection efficiency. Mn diffusion is also consistent with the difference in electrical properties of spin-LEDs with $\delta$-MnGa[151] and $\mathrm{Fe}$ contacts.

Figure 34 shows [1-10] and [110] cross-section HAADF-STEM images of a $\mathrm{Co}_{2} \mathrm{MnSi} / \mathrm{GaAs}(001)$ heterostructure. The images suggest that the interfaces look atomically abrupt, but detailed elemental atomic distribution at the interface may be complex, requiring a detailed comparison between theory and experiment. There is significant intensity modulation in the atomic rows on either side of the apparent interface. The $\mathrm{Co}_{2} \mathrm{MnSi}$ also shows regions of both $\mathrm{L} 2{ }_{1}$ and $\mathrm{B} 2$ ordering.

Non local spin valve measurements were performed on $\mathrm{Co}_{2} \mathrm{MnSi} / \mathrm{GaAs}(001)$ heterostructures using similar procedures to those used for $\mathrm{Fe}_{3} \mathrm{Ga} / \mathrm{GaAs}$ and $\mathrm{Fe} / \mathrm{GaAs}(001)[136]$. The spin valve signals for a $\mathrm{Co}_{2} \mathrm{MnSi} / \mathrm{GaAs}(001)$ and $\mathrm{Fe} / \mathrm{GaAs}(001)$ heterostructure are shown in Fig. 35(a) for both an unbiased and biased detector configuration. The temperature dependence of the spin signal for a biased detector (Fig. 35(b)) shows spin signal in excess of $2 \mathrm{mV}$ at low temperature. The non-local voltage is much larger for the $\mathrm{Co}_{2} \mathrm{MnSi}$ sample. By modeling the spin injection efficiency and enhancement due to detector bias, the spin accumulation in the GaAs channel was calculated to be $60 \%$ for $\mathrm{Co}_{2} \mathrm{MnSi}$ as compared to $30 \%$ for best $\mathrm{Fe}$ contacts[152].

The importance of the VEC to the properties of Heusler compounds was discussed in detail above. Fe has one more electron than Mn. Hence if Fe were substituted for Mn, the simple picture is that the Fermi level in the $\mathrm{Co}_{2} \mathrm{Mn}_{1-\mathrm{x}} \mathrm{Fe}_{\mathrm{x}} \mathrm{Si}$ would increase with $\mathrm{x}$. This is schematically depicted in Fig. 36 (top). The spin polarization at the Fermi level should decrease and switch sign when increasing $\mathrm{x}$ from 0 to 1 . This is what is experimentally observed (Fig. 36 (bottom)). The spin polarization has the opposite sign for $\mathrm{Co}_{2} \mathrm{FeSi}$ contacts as compared to $\mathrm{Co}_{2} \mathrm{MnSi}$.

The large spin signals obtained electrically from lateral $\mathrm{GaAs}$ spin devices with $\mathrm{Co}_{2} \mathrm{MnSi}$ and $\mathrm{Co}_{2} \mathrm{FeSi}$ ferromagnetic contacts have enabled detection of electron spin accumulation, electrically in GaAs up to room temperature [102,153]. Figure 37 shows the non local spin valve 
signal for $\mathrm{Co}_{2} \mathrm{MnSi} / \mathrm{GaAs}(001)$ as a function of contact separation for temperatures ranging from 25 to $300 \mathrm{~K}$.

$\mathrm{Co}_{2} \mathrm{MnSi}$ and $\mathrm{Co}_{2} \mathrm{FeSi}$ contacts have also been used to demonstrate tunneling anisotropic magnetoresistance (TAMR) detection of ferromagnetic resonance at room temperature even under conditions where no spin accumulation is present and no spin-polarized current flows in the semiconductor[154].

\section{Outlook}

Heusler based MTJs have been very successful and are of great technological interest for developing spin transfer torque magnetic random access memory (STT-RAM) devices $[155,156]$. This requires ferromagnetic material with spin torque efficiency and high TMR as well as low magnetic damping [156]. Perpendicular magnetic anisotropy reduces the switching current while maintaining thermal stability. Half-metallic Heusler compounds would have high TMR and high spin torque efficiency. The lack of a minority spin density of states at the Fermi level would also result in low magnetic damping. $\mathrm{NiMnSb}$ and $\mathrm{Co}_{2} \mathrm{MnSi}$ have very low magnetic damping coefficients $\left(\alpha=0.002\right.$ for $\mathrm{NiMnSb}$ [157] and 0.0007 for $\mathrm{Co}_{1.9} \mathrm{Mn}_{1.1} \mathrm{Si}$ [46], respectively). Interface induced perpendicular magnetic anisotropy using $\mathrm{MgO}(001)$ has been observed for $\mathrm{Co}_{2} \mathrm{FeAl}[158,159]$ and $\mathrm{Co}_{2} \mathrm{FeSi}$ [160] thin films. The challenge is to develop halfmetal Heuslers with bulk magnetic anisotropy rather than interface induced[156,161]. Azadani et al. have predicted a number of different Heusler superlattices depending on crystallographic orientation that should be both half-metallic and have perpendicular magnetic anisotropy [162]. These predictions have yet to be realized experimentally.

The versatility of Heusler compounds is shown by the vast number of properties they can have, the demonstration of MBE growth of high quality semiconducting [163,164], topological Heusler compounds [165-167], and the ability to tune their properties by controlling the VEC. The possibility to form heterostructures of Heusler compounds with vastly different electronic properties but with the same basic crystal structure and their demonstrated ability to integrate with conventional semiconductors and metal oxides promises an exciting future for their use in novel devices with new function.

\section{Acknowledgements}

The author would like to thank some of the collaborators in this area who have made this work so exciting and enjoyable: Johan Adell, Christoph Adelmann, Tobias Brown-Heft, William Butler, David Carr, Athanasios Chantis, Liang-Chin Chen, Kevin Christie, Scott Crooker, Paul Crowell, Jian Wei Dong, X.Y (Shirley) .Dong, Helen Farrell, Michael Flatté, Soren Flexner, Madalina Furis, Eric Garlid, Chad Geppert, Nickolas Harmon, Sean Harrington, Masahiko Hashimoto, Jessica Hilton, Qi Hu, Anderson Janotti, Jason Kawasaki, Changjiang Liu, Jay Logan, Kathy. Lüdge, Xiaohua Luo, Anthony McFadden, Anders Mikkelsen, Sahil Patel, Michael Pechan, Mihir Pendharkar, Timothy Peterson, Craig Polley, Madhukar Reddy, Anthony Rice, Brian Schultz, Tiffany Shih, Swadesh Srivastava, Gordon Stecklein, Balasubramanian Thiagarajan, Paul Voyles, Nathan Wilson, and Jianjie Zhang. The work in this review has been supported in part by C-SPIN, one of the six centers of STARnet, a Semiconductor Research Corporation program sponsored by MARCO and DARPA; by the NSF MRSEC program (NSF 
DMR-0212032); by the Office of Naval Research (ONR N0014-05-0208); by the Army Research Office (W911NF-12-1-0459); and by the NSF (DMR-1104951). 


\section{Figures}

Fig. 1 Periodic table with color coding showing the typical elements for X (blue), Y (red) and Z (green) in the half-Heusler XYZ and full-Heusler $\mathrm{X}_{2} \mathrm{YZ}$ compounds.

Fig. 2 General ordered cubic crystal structures for the full-Heuslers $\mathrm{X}_{2} \mathrm{YZ}$, the $\mathrm{D}_{3}$, when $\mathrm{X}$ and $\mathrm{Y}$ are the same element $\left(\mathrm{X}_{2} \mathrm{XZ}\right)$, the $\mathrm{B} 2$ when $\mathrm{Y}$ and $\mathrm{Z}$ are completely disordered, the half-Heuslers XYZ, the zincblende structure common to III-V semiconductors, and the $\mathrm{NaCl}$. Note the strong similarities between these structures.

Fig. 3 Electronic properties of full $(\mathrm{FH})$ and half $(\mathrm{HH})$ Heusler compounds as a function of the number of valence electrons per formula unit.

Fig. 4 Calculated total densities-of-states of half-Heusler compounds with various numbers of valence electrons per formula unit (from ref. [24]). Note how the Fermi level moves across the gap in the density of states with increasing valence electron count.

Fig. 5 Plot of spin magnetic moment versus valence electron count for a number half-Heusler compounds (from reference [36]).

Fig. 6 Plot of total magnetic moment versus valence electron count for a number full-Heusler compounds (from reference [5]).

Fig. 7 Slater-Pauling behavior for a number of inverse Heusler compounds. Note the rules of "18, 24 and 28" (from reference [27]).

Fig. 8 Slater-Pauling curve for a number of conventional magnetic alloys and some full-Heusler compounds (from ref [1]).

Fig. 9 Curie temperature versus VEC for a number of full Heusler compounds (from reference [1])

Fig. 10 Calculated spin dependent band structure of for the half metal $\mathrm{Co}_{2} \mathrm{MnSi}$ : a) density of states and b) majority and minority spin band structure (from reference [38]).

Fig. 11 Spin-resolved photoelectron spectroscopy spectra at $300 \mathrm{~K}$ for a series of $\mathrm{Co}_{2} \mathrm{Mn}_{1+\delta} \mathrm{Si}$ layers with increasing Mn content. As shown in the schemes, Fermi level is moving towards the spin polarization maximum as the Mn content increases. (from reference [46]).

Fig. 12 Influence of $\mathrm{Mn}$ and $\mathrm{MgO}$ coverage on $\mathrm{Co}_{2} \mathrm{MnSi}$ electronic properties. In (a) and (b) are shown the electron diffraction patterns for the starting $\mathrm{Co}_{2} \mathrm{MnSi}$ layers and when covered by $\mathrm{Mn}$ or $\mathrm{MgO}$. The Spin-resolved photoelectron spectroscopy spectra and spinpolarization spectra for the starting $\mathrm{Co}_{2} \mathrm{MnSi}$ layers and after the capping are shown in (c) Mn cap and (d) $\mathrm{MgO}$ cap. As the spin polarization is close to zero at Fermi level for both 
$\mathrm{Co}_{2} \mathrm{MnSi}$ uncoated surfaces, it is strongly increased when covering with $\mathrm{Mn}$ or $\mathrm{MgO}$. (from reference [46]).

Fig. 13 Schematic of MTJ and (b) spin valve (GMR) structures and density of states for parallel and antiparallel alignment of the ferromagnetic layers. In the antiparallel state there are no states for the majority spins (spin up) to go into in the antiparallel aligned electrode without spin flip scattering.

Fig. 14 Tunneling density of states for $\mathrm{k}_{\|}=0$ for $\mathrm{Fe}(100) / \mathrm{MgO} / \mathrm{Fe}(100)$ for majority and minority spins with aligned (upper panels) and antialigned (lower panels) magnetic moments (from reference [52]).

Fig. 15 TMR versus date for full Heusler compound magnetic tunnel junctions. The data were obtained from references [57-67].

Fig. $16 \mathrm{TMR}$ ratio for $\mathrm{Co}_{2}(\mathrm{Mn}, \mathrm{Fe})_{\mathrm{y}} \mathrm{Si}_{0.84}$ dependence on the $\mathrm{Y}$ site concentration $(\mathrm{Mn}+\mathrm{Fe})$ (a) and (b) and VEC (c) and (d). (Data obtained from reference [67]).

Fig. 17 HAADF-STEM images of a $\mathrm{Co}_{2} \mathrm{Mn}_{1.24} \mathrm{Fe}_{0.16} \mathrm{Si}_{0.84} / \mathrm{MgO} / \mathrm{Co}_{2} \mathrm{Mn}_{1.24} \mathrm{Fe}_{0.16} \mathrm{Si}_{0.84} \mathrm{MTJ}$. Regions (b) and (c) correspond to areas with $\mathrm{B} 2$ and $\mathrm{L} 2{ }_{1}$ crystal structures, respectively. Note that the lower $\mathrm{MgO} / \mathrm{Co}_{2} \mathrm{Mn}_{1.24} \mathrm{Fe}_{0.16} \mathrm{Si}_{0.84}$ interface is substantially smoother than the upper interface. (from reference [67]).

Fig. 18 Spin-FET. The Schottky gate is used to control the spin precession as electrons in a 2DEG are transported from the spin injector to spin detector. Semiconductors with large spin-orbit coupling will have more spin precession

Fig. 19 Plot of lattice parameter versus element for a number of Heusler compounds. Note how the lattice parameters span those of a number of III-V compound semiconductors. The effective lattice parameter for $\mathrm{MgO}$ assuming a 45 degree rotation $\left(\sqrt{2} \mathrm{a}_{\mathrm{MgO}}\right)$ is also indicated.

Fig. 20 Predicted Fe-Ga-As ternary phase diagram. (adapted from reference [86]).

Fig. $21 \mathrm{GaAs}(001)$ surface reconstruction phase diagram (from reference [129]).

Fig. 22 Schematic a spin-LED (a) band diagram, (b) typical heterostructure and (c) electroluminescence polarization as a function of out of plane magnetic field for a spinLED with ferromagnetic $\mathrm{Fe}_{3} \mathrm{Ga}$ spin injection contact.

Fig. 23 Schematic diagrams of the experiment and representative non-local spin-valve and Hanle effects. (a) typical layer structure beneath the ferromagnetic contacts. Note that the $\mathrm{n}^{+}-$ GaAs layers are etched away in the transport channel regions. (b) band diagram of the Schottky contact with the $\mathrm{n}^{+}$-doped GaAs contact layers, (c) non-local spin-valve device structure, (d) and (e) are the non-local spin valve and Hanle effect measurements. The 
upper panels show the raw data and the lower panels after background subtraction. The inserts show the measurement configurations.

Fig. $24 \mathrm{X}$-ray diffraction scan, rocking curve and reciprocal space map of a $\mathrm{Al}$ capped $\mathrm{Fe}_{3} \mathrm{Ga}(30 \mathrm{~nm}) / \mathrm{GaAs}(001)$ heterostructure grown by codeposition at $325^{\circ} \mathrm{C}$.

Fig. 25 Considerations on the growth procedure for $\mathrm{Fe}_{3} \mathrm{Ga}$ on $\mathrm{GaAs}(001)$.

Fig. 26 Procedures used to create different $\mathrm{Fe}_{3} \mathrm{Ga} / \mathrm{GaAs}(001)$ interfaces.

Fig. 27 HAADF-STEM images of $\mathrm{Fe}_{3} \mathrm{Ga} / \mathrm{GaAs}(001)$ heterostructure interfaces: top: Sample E with $\mathrm{Fe} / \mathrm{As}$ interface grown at $175^{\circ} \mathrm{C}$ on $\mathrm{GaAs}(2 \times 4) / \mathrm{c}(2 \times 8)$ surface and bottom Sample A - $\mathrm{FeGa} / \mathrm{Ga}$ interface grown at $300^{\circ} \mathrm{C}$ on $\mathrm{GaAs}(4 \times 6) / \mathrm{c}(8 \times 2)$ surface) (from [140])

Fig. 28 Bias dependent lateral spin valve signals for $\mathrm{Fe}_{3} \mathrm{Ga} / \mathrm{GaAs}(001)$ heterostructures grown with different interface growth sequences. The Fe-Ga intiated growth on a Ga-rich $\mathrm{GaAs}(001)$ surface shows the highest spin valve signal (from [140])

Fig. $295 \mathrm{~nm}$ thick $\mathrm{Co}_{2} \mathrm{MnSi}$ grown on GaAs (001) (a) RHEED diffraction patterns, (b) RHEED intensity oscillations during growth, (c) in-situ STM image of the surface and (d) ex-situ magnetic hysteresis loops. (adapted from reference [141])

Fig. 30 RHEED of a starting c(4x4) GaAs (001) surface as well as after the deposition of each monolayer in Co- and $\mathrm{MnSi}$-initiated samples of $\mathrm{Co}_{2} \mathrm{MnSi}$.

Fig. 31 Measured intensities of the Ga 3d, Co 2p and Mn 2p core levels as a function of sequential monolayer growth thickness. Top panels: MnSi initiated and bottom panel Co initiated films. Note that the Co $2 \mathrm{p}$ peak intensity does not decrease after the first $\mathrm{MnSi}$ monolayer deposition for the Co initiated film.

Fig. 32 Schematic of the results from the sequential monolayer growth studies. The $\mathrm{Co}_{2} \mathrm{MnSi} / \mathrm{GaAs}$ is similar regardless of the nucleation sequence.

Fig. $33 \mathrm{Co}_{2} \mathrm{MnSi} / \mathrm{GaAs}(001)$ Schottky contacts (a) Current-voltage characteristics at room temperature for $\mathrm{Si}, \mathrm{Mn}, \mathrm{Co}$ and $\mathrm{MnSi}$ initiated growth. (b) Backside SIMS depth profile to detect preferential $\mathrm{Mn}$ indiffusion beneath the contact.

Fig. 34 Cross-sectional HAADF-STEM images of $\mathrm{Co}_{2} \mathrm{MnSi} / \mathrm{GaAs}(001)$ interface. (Courtesy of P. Voyles).

Fig. 35 Lateral transport non local spin valve signals for $\mathrm{Co}_{2} \mathrm{MnSi} / \mathrm{GaAs}(001)$ and $\mathrm{Fe} / \mathrm{GaAs}(001)$ for (a) unbiased and biased detector. (b) temperature dependence of the spin signal for a $\mathrm{Co}_{2} \mathrm{MnSi} / \mathrm{GaAs}(001)$ with a biased detector (Courtesy P. Crowell). 
Fig. 36 Top: Schematic of the density of states for $\mathrm{Co}_{2} \mathrm{Mn}_{1-\mathrm{x}} \mathrm{Fe}_{\mathrm{x}} \mathrm{Si}$ as a function of $\mathrm{x}$. Bottom: Temperature dependence of spin polarization determined by biased detector technique (Courtesy P. Crowell).

Fig. 37 Non-local voltage versus $\mathrm{Co}_{2} \mathrm{MnSi}$ contact separation for 25-300K (Courtesy P. Crowell). 


\section{References}

1 T. Graf, C. Felser, and S. S. P. Parkin, Progress in Solid State Chemistry 39, 1 (2011).

2 Z. Xiuwen, Y. Liping, A. Zakutayev, and A. Zunger, Advanced Functional Materials 22, 1425 (2012).

3 A. Zakutayev, X. Zhang, A. Nagaraja, L. Yu, S. Lany, T. O. Mason, D. S. Ginley, and A. Zunger, Journal of the American Chemical Society 135, 10048 (2013).

4 D. Jung, H. J. Koo, and M. H. Whangbo, Journal of Molecular Structure-Theochem 527, 113 (2000).

$5 \quad$ I. Galanakis, P. H. Dederichs, and N. Papanikolaou, Phys. Rev. B 66, 174429 (2002).

$6 \quad$ I. Galanakis, P. H. Dederichs, and N. Papanikolaou, Phys. Rev. B 66, 134428 (2002).

$7 \quad$ H. C. Kandpal, C. Felser, and R. Seshadri, J. Phys. D-Appl. Phys. 39, 776 (2006).

L. Offernes, P. Ravindran, and A. Kjekshus, Journal of Alloys and Compounds 439, 37 (2007).

J. Tobola and J. Pierre, Journal of Alloys and Compounds 296, 243 (2000).

10 X. Y. Dong, J. W. Dong, J. Q. Xie, T. C. Shih, S. McKernan, C. Leighton, and C. J. Palmstrøm, J. Crystal Growth 254, 384 (2003).

11 R. A. de Groot, F. M. Mueller, P. G. van Engen, and K. H. J. Buschow, Phys. Rev. Letts. 50, 2024 (1983).

12 S. Ögöt and K. M. Rabe, Physical Review B 51, 10443 (1995).

13 M. Meinert, M. P. Geisler, J. Schmalhorst, U. Heinzmann, E. Arenholz, W. Hetaba, M. StoegerPollach, A. Huetten, and G. Reiss, Physical Review B 90, 085127 (2014).

14 S. Yabuuchi, M. Okamoto, A. Nishide, Y. Kurosaki, and J. Hayakawa, Applied Physics Express 6, 025504 (2013).

15 J. Winterlik, G. H. Fecher, and C. Felser, Solid State Communications 145, 475 (2008).

16 T. Klimczuk, C. H. Wang, K. Gofryk, F. Ronning, J. Winterlik, G. H. Fecher, J. C. Griveau, E. Colineau, C. Felser, J. D. Thompson, D. J. Safarik, and R. J. Cava, Physical Review B 85, 174505 (2012).

17 Y. Pan, A. M. Nikitin, T. V. Bay, Y. K. Huang, C. Paulsen, B. H. Yan, and A. de Visser, Epl 104, 27001 (2013).

18 G. Xu, W. Wang, X. Zhang, Y. Du, E. Liu, S. Wang, G. Wu, Z. Liu, and X. X. Zhang, Scientific Reports 4, 5709 (2014).

19 F. F. Tafti, T. Fujii, A. Juneau-Fecteau, S. R. de Cotret, N. Doiron-Leyraud, A. Asamitsu, and L. Taillefer, Physical Review B 87, 184504 (2013).

20 A. Roy, J. W. Bennett, K. M. Rabe, and D. Vanderbilt, Physical Review Letters 109, 037602 (2012).

21 Half-metallic Alloys. Fundamentals and Applications, Lecture Notes in Physics, Vol. 676, Edited by I. Galanakis and P. H. Dederichs, (Springer, Berlin Heidelberg, 2005).

22 T. Graf, J. Winterlik, L. Muechler, G. H. Fecher, C. Felser, and S. S. P. Parkin, in Handbook of Magnetic Materials, Vol 21; Vol. 21, edited by K. H. J. Buschow (2013), p. 1.

23 C. Felser, L. Wollmann, S. Chadov, G. H. Fecher, and S. S. P. Parkin, APL Materials 3, 041518 (2015).

24 J. Tobola, J. Pierre, S. Kaprzyk, R. V. Skolozdra, and M. A. Kouacou, Journal of PhysicsCondensed Matter 10, 1013 (1998).

25 J. C. Slater, Physical Review 49, 537 (1936).

26 L. Pauling, Physical Review 54, 899 (1938).

S. Skaftouros, K. Ozdogan, E. Sasioglu, and I. Galanakis, Physical Review B 87, 024420 (2013). I. Galanakis, S. Ostanin, M. Alouani, H. Dreysse, and J. M. Wills, Physical Review B 61, 4093 (2000).

29 J. Kübler, A. R. Williams, and C. B. Sommers, Phys. Rev. B 28, 1745 (1983). 
S. Fujii, S. Sugimura, Ishida, and S. Asano, J. Phys.:-Condens. Matter 2, 8583 (1990).

S. Fujii, S. Ishida, and S. Asano, Journal of the Physical Society of Japan 64, 185 (1995).

S. Ishida, S. Fujii, S. Kashiwagi, and S. Asano, Journal of the Physical Society of Japan 64, 2152 (1995).

T. Block, C. Felser, G. Jakob, J. Ensling, B. Muhling, P. Gutlich, and R. J. Cava, Journal of Solid State Chemistry 176, 646 (2003).

H. C. Kandpal, G. H. Fecher, and C. Felser, J. Phys. D-Appl. Phys. 40, 1507 (2007).

I. Galanakis and P. H. Dederichs, in Half-metallic Alloys Fundamentals and Applications, edited by I. Galanakis and P. H. Dederichs (Springer, Berlin Heidelberg, 2005), p. 1.

M. I. Katsnelson, V. Y. Irkhin, L. Chioncel, A. I. Lichtenstein, and R. A. de Groot, Reviews of Modern Physics 80, 315 (2008).

B. Balke, S. Ouardi, T. Graf, J. Barth, C. G. F. Blum, G. H. Fecher, A. Shkabko, A. Weidenkaff, and C. Felser, Solid State Communications 150, 529 (2010).

D. Orgassa, H. Fujiwara, T. C. Schulthess, and W. H. Butler, J. Appl. Phys. 87, 5870 (2000).

S. Picozzi, A. Continenza, and A. J. Freeman, Phys Rev B 69, 094423 (2004).

S. Picozzi, A. Continenza, and A. J. Freeman, Half-Metallic Alloys: Fundamentals and Applications 676, 41 (2005).

K. Ozdogan and I. Galanakis, J. Appl. Phys. 110, 076101 (2011).

I. Galanakis, Journal of Physics-Condensed Matter 14, 6329 (2002).

S. J. Hashemifar, P. Kratzer, and M. Scheffler, Physical Review Letters 94, 096402 (2005).

Z. Zhu, H. Wang, and U. Schwingenschloegl, Advanced Materials Interfaces 2, 1400340 (2015).

S. Andrieu, A. Neggache, T. Hauet, T. Devolder, A. Hallal, M. Chshiev, A. Bataille, P. Le Févre, and F. Bertran, Phys Rev B 93, 094417 (2016).

J.-P. Wuestenberg, R. Fetzer, M. Aeschlimann, M. Cinchetti, J. Minar, J. Braun, H. Ebert, T. Ishikawa, T. Uemura, and M. Yamamoto, Physical Review B 85, 064407 (2012).

M. Jourdan, J. Minar, J. Braun, A. Kronenberg, S. Chadov, B. Balke, A. Gloskovskii, M. Kolbe, H. J. Elmers, G. Schoenhense, H. Ebert, C. Felser, and M. Klaeui, Nature Communications 5, 3974 (2014).

S. Picozzi, A. Continenza, and A. J. Freeman, J. Appl. Phys. 94, 4723 (2003). M. Julliere, Physics Letters A 54, 225 (1975).

J. M. D. Coey, in Magnetism and magnetic materials (Cambridge Univeristy Press, Cambridge, UK, 2010), p. 494.

W. H. Butler, X. G. Zhang, T. C. Schulthess, and J. M. MacLaren, Physical Review B-Condensed Matter 63, 054416/1 (2001).

J. Mathon and A. Umerski, Phys Rev B 63, 220403 (2001).

S. S. P. Parkin, C. Kaiser, A. Panchula, P. M. Rice, B. Hughes, M. Samant, and S.-H. Yang, Nature Materials 3, 862 (2004).

S. Yuasa, T. Nagahama, A. Fukushima, Y. Suzuki, and K. Ando, Nature Materials 3, 868 (2004).

S. Yuasa and D. D. Djayaprawira, J. Phys. D-Appl. Phys. 40, R337 (2007).

S. Kämmerer, A. Thomas, A. Hütten, and G. Reiss, Appl. Phys.Lett. 85, 79 (2004).

S. Okamura, A. Miyazaki, S. Sugimoto, N. Tezuka, and K. Inomata, Appl. Phys. Lett. 86, 232503 (2005).

Y. Sakuraba, J. Nakata, M. Oogane, H. Kubota, Y. Ando, A. Sakuma, and T. Miyazaki, Japanese Journal of Applied Physics Part 2-Letters \& Express Letters 44, L1100 (2005).

N. Tezuka, N. Ikeda, A. Miyazaki, S. Sugimoto, M. Kikuchi, and K. Inomata, Appl. Phys. Lett. 89, 112514 (2006).

Y. Sakuraba, M. Hattori, M. Oogane, Y. Ando, H. Kato, A. Sakuma, T. Miyazaki, and H. Kubota, Appl. Phys. Lett. 88, 192508 (2006).

62 N. Tezuka, N. Ikeda, S. Sugimoto, and K. Inomata, Japanese Journal of Applied Physics, Part 2 (Letters) 46, L454 (2007). 

Condensed Matter 22, 164212 (2010). Phys. Lett. 101, 132418 (2012).

67 H.-x. Liu, T. Kawami, K. Moges, T. Uemura, M. Yamamoto, F. Shi, and P. M. Voyles, J. Phys. D-Appl. Phys. 48, 164001 (2015).

68 S. Datta and B. Das, Appl. Phys. Lett. 56, 665 (1990).

69 M. Holub, J. Shin, D. Saha, and P. Bhattacharya, Physical Review Letters 98, 146603 (2007). P. Bhattacharya, D. Basu, A. Das, and D. Saha, Semicond. Sci. Technol. 26, 014002 (2011). I. Žutić, R. Oszwaldowski, J. Lee, and C. Gøthgen, in Hanbook of Spin Transport and Magnetism, edited by E. Y. Tsymbal and I. Žutić (CRC Press, Taylor \& Francis Group, Boca Raton, Florida, 2012), p. 731. H. Dery, in Hanbook of Spin Transport and Magnetism, edited by E. Y. Tsymbal and I. Žutić (CRC Press, Taylor \& Francis Group, Boca Raton, Florida, 2012), p. 747. C. J. Palmstrøm, Annu. Rev. Mater. Sci. 25, 389 (1995).

R. Beyers, K. B. Kim, and R. Sinclair, J. Appl. Phys. 61, 2195 (1987).

R. Schmid-Fetzer, J. Electronic Mater. 17, 193 (1988). C. J. Palmstrøm and D. V. Morgan, in Gallium Arsenide Materials, Devices, and Circuits, edited by M. J. Howes and D. V. Morgan (John Wiley \& Sons Ltd., Chichester, 1985), p. 195. (1987). Ramesh, and Y. Silberberg, Materials Science Reports 5, 99 (1990). edited by L. J. Brillson (Noyes, Park Ridge, NJ, 1993), p. 67. A. Lahav, M. Eizenberg, and Y. Komem, J. Appl. Phys 60, 991 (1986). J. L. Hilton, B. D. Schultz, S. McKernan, S. M. Spanton, M. M. R. Evans, and C. J. Palmstrøm, J. Vac. Sci. Technol. B 23, 1752 (2005). A. J. Yu, G. J. Galvin, C. J. Palmstrøm, and J. W. Mayer, Appl. Phys. Lett. 47, 934 (1985). M. Genut and M. Eizenberg, Appl. Phys. Lett. 50, 1358 (1987). C. J. Palmstrøm, C. C. Chang, A. Yu, G. J. Galvin, and J. W. Mayer, J. Appl. Phys. 62, 3755 (1987). Science \& Technology B 20, 1600 (2002). 92, 091914 (2008). M. Hong, H. S. Chen, J. Kwo, A.
Cryst. Growth 111, 984 (1991). Appl. Phys. 73, 6766 (1993). J. Herfort, H.-P. Schonherr, K.-J. Friedland, and K. H. Ploog, Journal of Vacuum Science \& Technology B (Microelectronics and Nanometer Structures) 22, 2073 (2004).

90 J. W. Dong, L. C. Chen, C. J. Palmstrøm, R. D. James, and S. McKernan, Appl. Phys. Lett. 75, 1443 (1999).

91 J. W. Dong, L. C. Chen, J. Q. Xie, T. A. R. Muller, D. M. Carr, C. J. Palmstrøm, S. McKernan, Q. Pan, and R. D. James, J. Appl. Phys. 88, 7357 (2000).

92 J. Q. Xie, J. W. Dong, J. Lu, C. J. Palmstrøm, and S. McKernan, Appl. Phys. Lett. 79, 1003 (2001). 

80, 4798 (2002).

94 J. Q. Xie, J. Lu, J. W. Dong, X. Y. Dong, T. C. Shih, S. McKernan, and C. J. Palmstrøm, J. Appl. Phys. 97, 073901 (2005).

95 X. Y. Dong, C. Adelmann, J. Q. Xie, C. J. Palmstrøm, X. Lou, J. Strand, P. A. Crowell, and A. K. Petford-Long, Appl. Phys. Lett. 86, 102107 (2005).

96 S. Bohse, A. Zolotaryov, A. Volland, B. Landgraf, O. Albrecht, M. Bastjan, T. Vossmeyer, D. Goerlitz, C. Heyn, and W. Hansen, Journal of Crystal Growth 338, 91 (2012).

97 T. Ambrose, J. J. Krebs, and G. A. Prinz, Appl. Phys. Lett. 76, 3280 (2000).

98 T. Ambrose, J. J. Krebs, and G. A. Prinz, J. Appl. Phys. 89, 7522 (2001).

99 F. Y. Yang, C. H. Shang, C. L. Chien, T. Ambrose, J. J. Krebs, G. A. Prinz, V. I. Nikitenko, V. S. Gornakov, A. J. Shapiro, and R. D. Shull, Physical Review B-Condensed Matter 65, 174410/1 (2002).

100 Growth and magnetotransport properties of thin $\mathrm{Co}_{2} \mathrm{MnGe}$ layered structures, Half-Metallic Alloys. Fundamentals and Applications, Edited by I. Galanakis and P. H. Dederichs, (Springer, Berlin Heidelberg, 2005).

101 W. H. Wang, M. Przybylski, W. Kuch, L. I. Chelaru, J. Wang, Y. F. Lu, J. Barthel, H. L. Meyerheim, and J. Kirschner, Phys. Rev. B 71, 144416 (2005).

102 Y. Ebina, T. Akiho, H.-x. Liu, M. Yamamoto, and T. Uemura, Appl. Phys. Lett. 104, 172405 (2014).

103 M. Hashimoto, J. Herfort, A. Trampert, H.-P. Schonherr, and K. H. Ploog, Journal of Physics D (Applied Physics) 40, 1631 (2007).

104 M. Hashimoto, J. Herfort, A. Trampert, H.-P. Schonherr, and K. H. Ploog, Journal of Vacuum Science \&amp; Technology B (Microelectronics and Nanometer Structures) 24, 2004 (2006).

105 M. Ramsteiner, O. Brandt, T. Flissikowski, H. T. Grahn, M. Hashimoto, J. Herfort, and H. Kostial, Physical Review B 78, (2008).

106 H. Lehmann, J. M. Scholtyssek, C. Herrmann, J. Herfort, U. Merkt, and G. Meier, J. Appl. Phys. 110, 063908/1 (2011).

107 M. J. Pechan, C. Yu, D. Carr, and C. J. Palmstrøm, J. Magnetism and Magnetic Materials 286, 340 (2005).

108 T. C. Shih, J. Q. Xie, J. W. Dong, X. Y. Dong, S. Srivastava, C. Adelmann, S. McKernan, R. D. James, and C. J. Palmstrøm, Ferroelectrics 343, 35 (2006).

109 W. Van Roy, J. De Boeck, B. Brijs, and G. Borghs, Appl. Phys. Lett. 77, 4190 (2000).

110 W. Van Roy, G. Borghs, and J. De Boeck, Journal of Magnetism \& Magnetic Materials 242-245, 489 (2002).

111 P. Bach, A. S. Bader, C. Ruster, C. Gould, C. R. Becker, G. Schmidt, L. W. Molenkamp, W. Weigand, C. Kumpf, E. Umbach, R. Urban, G. Woltersdorf, and B. Heinrich, Appl. Phys. Lett. 83, 521 (2003).

112 A. Koveshnikov, G. Woltersdorf, J. Q. Liu, B. Kardasz, O. Mosendz, B. Heinrich, K. L. Kavanagh, P. Bach, A. S. Bader, C. Schumacher, C. Ruster, C. Gould, G. Schmidt, L. W. Molenkamp, and C. Kumpf, J. Appl. Phys. 97, (2005).

113 W. Van Roy and M. Wojcik, in Half-Metallic Alloys: Fundamentals and Applications; Vol. 676, edited by I. Galanakis and P. H. Dederichs (Springer, Berlin Heidelberg, 2005), p. 153.

114 B. Botters, F. Giesen, J. Podbielski, P. Bach, G. Schmidt, L. W. Molenkamp, and D. Grundler, Appl. Phys. Lett. 89, (2006).

115 L. Makinistian, M. M. Faiz, R. P. Panguluri, B. Balke, S. Wurmehl, C. Felser, E. A. Albanesi, A. G. Petukhov, and B. Nadgorny, Physical Review B 87, 220402 (2013).

116 J. M. Scholtyssek, L. Bocklage, R. Anton, U. Merkt, and G. Meier, Journal of Magnetism and Magnetic Materials 316, E923 (2007).

117 L. Bocklage, J. M. Scholtyssek, U. Merkt, and G. Meier, J. Appl. Phys. 101, 09J512 (2007). 
R. J. J. Soulen, J. M. Byers, M. S. Osofsky, B. Nadgorny, T. Ambrose, S. F. Cheng, P. R. Broussard, C. T. Tanaka, J. Nowak, J. S. Moodera, A. Barry, and J. M. D. Coey, Science 282, 85 (1998).

119 Y. Miyoshi, Y. Bugoslavsky, M. H. Syed, T. Robinson, L. F. Cohen, L. J. Singh, Z. H. Barber, C. E. A. Grigorescu, S. Gardelis, J. Giapintzakis, and W. Van Roy, Appl. Phys. Lett. 88, 142512 (2006).

120 K. R. A. Ziebeck and P. J. Webster, Journal of Physics F-Metal Physics 5, 1756 (1975).

121 M. Acet, E. Duman, E. F. Wassermann, L. Manosa, and A. Planes, J. Appl. Phys. 92, 3867 (2002).

122 G. Schmidt, D. Ferrand, L. W. Molenkamp, A. T. Filip, and B. J. van Wees, Phys. Rev. B 62, R4790 (2000).

123 A. T. Hanbicki, B. T. Jonker, G. Itskos, G. Kioseoglou, and A. Petrou, Appl. Phys. Lett. 80, 1240 (2002).

124 A. T. Hanbicki, O. M. J. van 't Erve, R. Magno, G. Kioseoglou, C. H. Li, B. T. Jonker, G. Itskos, R. Mallory, M. Yasar, and A. Petrou, Appl. Phys. Lett. 82, 4092 (2003).

125 Q. O. Hu, E. S. Garlid, P. A. Crowell, and C. J. Palmstrøm, Phys. Rev. B 84, 085306 (2011).

126 C. Adelmann, B. D. Schultz, J. Strand, X. Lou, X. Y. Dong, J. Q. Xie, S. Park, M. R. Fitzsimmons, P. A. Crowell, and C. J. Palmstrøm, in 2004 International Conference on Indium Phosphide and Related Materials (Kagoshima, Japan, 2004), p. 505.

127 C. Adelmann, J. Q. Xie, C. J. Palmstrøm, J. Strand, X. Lou, J. Wang, and P. A. Crowell, J. Vac. Sci. Technol. B 23, 1747 (2005).

128 B. D. Schultz, C. Adelmann, X. Lou, J. Strand, P. A. Crowell, N. Marom, D. Naveh, L. Kronik, and C. J. Palmstrøm, Phys. Rev. B (Rapid Comm.) 80, 201309(R) (2009).

129 L. Daweritz and R. Hey, Surface Science 236, 15 (1990).

130 A. Ohtake, Surface Science Reports 63, 295 (2008).

131 L. C. Chen, J. W. Dong, B. D. Schultz, C. J. Palmstrøm, J. Berezovsky, A. Isakovic, P. A. Crowell, and N. Tabat, J. Vac. Sci. Technol. B 18, 2057 (2000).

132 H. J. Zhu, M. Ramsteiner, H. Kostial, M. Wassermeier, H.-P. Schönherr, and K. H. Ploog, Phys. Rev. Lett 87, 016601 (2001).

133 V. F. Motsnyi, J. De Boeck, J. Das, W. Van Roy, G. Borghs, E. Goovaerts, and V. I. Safarov, Appl. Phys. Lett. 81, 265 (2002).

134 C. Adelmann, X. Lou, J. Strand, C. J. Palmstrøm, and P. A. Crowell, Phys Rev B 71, 121301(R) (2005).

135 X. Y. Dong, C. Adelmann, J. Q. Xie, C. J. Palmstrøm, X. Lou, J. Strand, P. A. Crowell, J. P. Barnes, and A. K. Petford-Long, Appl. Phys. Lett. 86, 102107/1 (2005).

136 X. Lou, C. Adelmann, S. A. Crooker, E. S. Garlid, J. Zhang, S. M. Reddy, S. D. Flexner, C. J. Palmstrøm, and P. A. Crowell, Nature Physics 3, 197 (2007).

137 P. A. Crowell and S. A. Crooker, in Handbook of Spin Transport and Magnetism, edited by E. Y. Tsymbal and I. Zutic (CRC Press, Taylor \& Francis Group, Boca Raton, FL, 2012), p. 463.

138 S. A. Crooker, M. Furis, X. Lou, C. Adelmann, D. L. Smith, C. J. Palmstrøm, and P. A. Crowell, Science 309, 2191 (2005).

139 S. A. Crooker, E. Garlid, A. N. Chantis, D. L. Smith, K. S. M. Reddy, Q. Hu, T. Kondo, C. J. Palmstrøm, and P. A. Crowell, Phys. Rev. B (Rapid Comm) 80, 041305/1 (2009).

140 M. Hashimoto, Q. O. Hu, E. S. Garlid, S. Krämer, P. A. Crowell, and C. J. Palmstrøm, Presented at PCSI-37, unpublished (2010).

141 S. J. Patel, Ph.D. Thesis, University of California, Santa Barbara, 2015.

142 S. Picozzi and A. J. Freeman, Journal of Physics-Condensed Matter 19, 315215 (2007).

143 K. Nagao, Y. Miura, and M. Shirai, Physical Review B 73, 104447 (2006).

144 L.-Y. Chen, S.-F. Wang, Y. Zhang, J.-M. Zhang, and K.-W. Xu, Thin Solid Films 519, 4400 (2011).

145 S. V. Eremeev, S. S. Kul'kov, and S. E. Kul'kova, Physics of the Solid State 50, 259 (2008). 
146 N. Ghaderi, S. J. Hashemifar, H. Akbarzadeha, and M. Peressi, J. Appl. Phys. 102, 074306 (2007).

147 S. Khosravizadeh, S. J. Hashemifar, and H. Akbarzadeh, Physical Review B 79, 235203 (2009).

148 I. Galanakis, Journal of Physics-Condensed Matter 16, 8007 (2004).

149 I. Galanakis, M. Lezaic, G. Bihlmayer, and S. Blugel, Physical Review B 71, 214431 (2005).

150 C. J. Palmstrøm, S. A. Schwarz, E. Yablonovitch, J. P. Harbison, C. L. Schwartz, L. T. Florez, T. J. Gmitter, E. D. Marshall, and S. S. Lau, J. Appl. Phys. 67, 334 (1990).

151 C. Adelmann, J. L. Hilton, B. D. Schultz, S. McKernan, C. J. Palmstrøm, X. Lou, H.-S. Chiang, and P. A. Crowell, Appl. Phys. Lett. 89, 112511 (2006).

152 P. A. Crowell, (2015).

153 C. Liu, S. J. Patel, T. A. Peterson, C. C. Geppert, K. D. Christie, G. Stecklein, C. J. Palmstrøm, and P. A. Crowell, Nature Communications 7, 10296 (2016).

154 C. Liu, Y. Boyko, C. C. Geppert, K. D. Christie, G. Stecklein, S. J. Patel, C. J. Palmstrøm, and P. A. Crowell, Appl. Phys. Lett. 105, 212401 (2014).

155 E. Chen, D. Apalkov, Z. Diao, A. Driskill-Smith, D. Druist, D. Lottis, V. Nikitin, X. Tang, S. Watts, S. Wang, S. A. Wolf, A. W. Ghosh, J. W. Lu, S. J. Poon, M. Stan, W. H. Butler, S. Gupta, C. K. A. Mewes, T. Mewes, and P. B. Visscher, Ieee Transactions on Magnetics 46, 1873 (2010).

156 K. Munira, J. Romero, and W. H. Butler, J. Appl. Phys. 115, $17 \mathrm{~b} 731$ (2014).

157 B. Pigeau, G. de Loubens, O. Klein, A. Riegler, F. Lochner, G. Schmidt, L. W. Molenkamp, V. S. Tiberkevich, and A. N. Slavin, Appl. Phys. Lett. 96, 132506 (2010).

158 Z. Wen, H. Sukegawa, S. Kasai, M. Hayashi, S. Mitani, and K. Inomata, Applied Physics Express 5, 063003 (2012).

159 Z. Wen, H. Sukegawa, S. Mitani, and K. Inomata, Appl. Phys. Lett. 98, 242507 (2011).

160 Y. Takamura, T. Suzuki, Y. Fujino, and S. Nakagawa, J. Appl. Phys. 115, 17c732 (2014).

161 J. Jeong, Y. Ferrante, S. V. Faleev, M. G. Samant, C. Felser, and S. S. P. Parkin, Nature Communications 7, 10276 (2016).

162 J. G. Azadani, K. Munira, J. Romero, J. Ma, C. Sivakumar, A. W. Ghosh, and W. H. Butler, J. Appl. Phys. 119, 043904 (2016).

163 J. K. Kawasaki, L. I. M. Johansson, B. D. Schultz, and C. J. Palmstrøm, Appl. Phys. Lett. 104, 022109 (2014).

164 J. K. Kawasaki, T. Neulinger, R. Timm, M. Hjort, A. A. Zakharov, A. Mikkelsen, B. D. Schultz, and C. J. Palmstrøm, Journal of Vacuum Science \& Technology B 31, 04 D106 (2013).

165 S. J. Patel, J. A. Logan, S. D. Harrington, B. D. Schultz, and C. J. Palmstrøm, Journal of Crystal Growth 436, 145 (2016).

166 J. A. Logan, S. J. Patel, S. D. Harrington, C. M. Polley, B. D. Schultz, T. Balasubranabian, A. Janotti, A. Mikkelsen, and C. J. Palmstrøm, ArXiv_1511.04778, (2015).

167 S. J. Patel, J. K. Kawasaki, J. Logan, B. D. Schultz, J. Adell, B. Thiagarajan, A. Mikkelsen, and C. J. Palmstrøm, Appl. Phys. Lett. 104, 201603 (2014). 


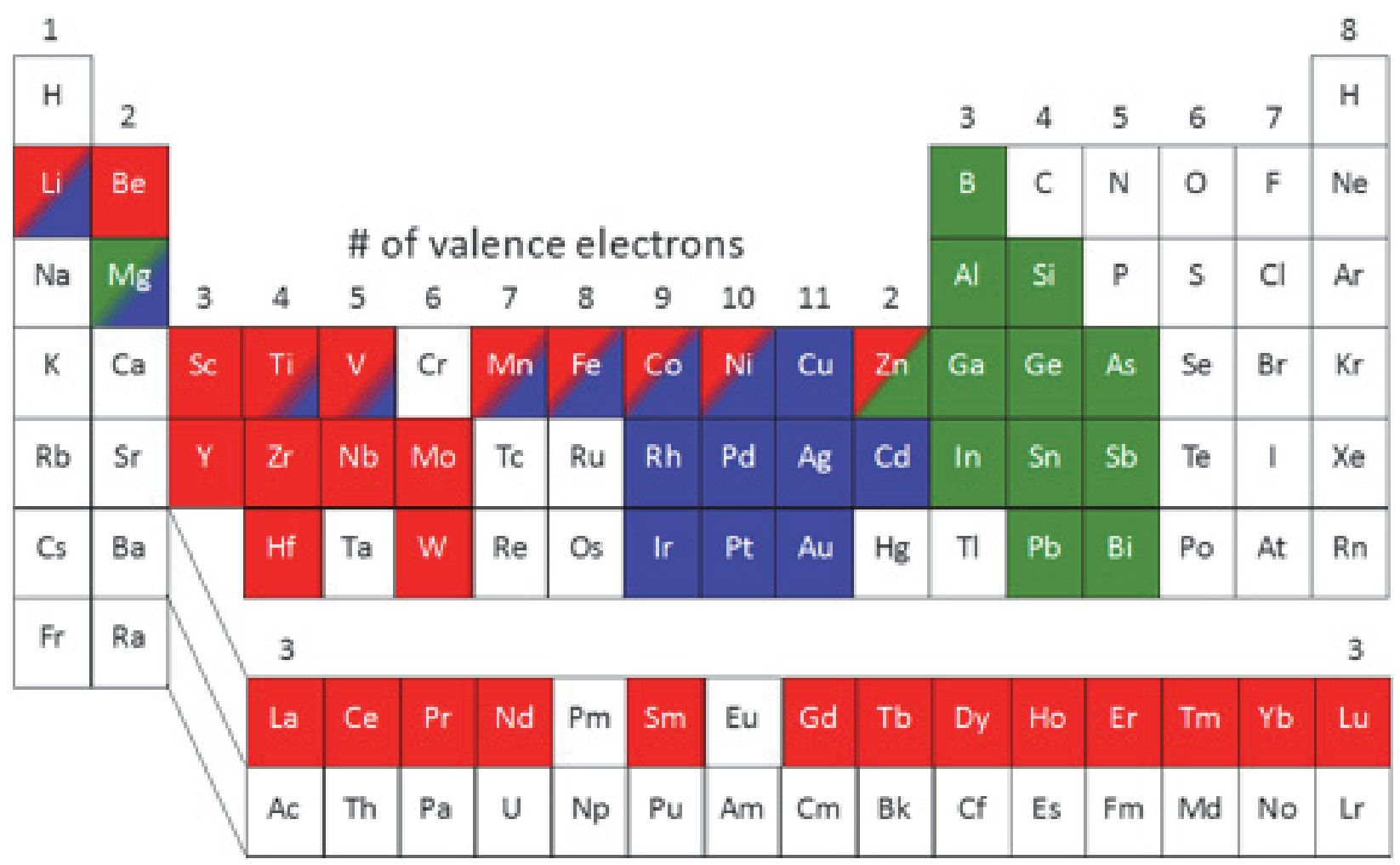

Fig.1 


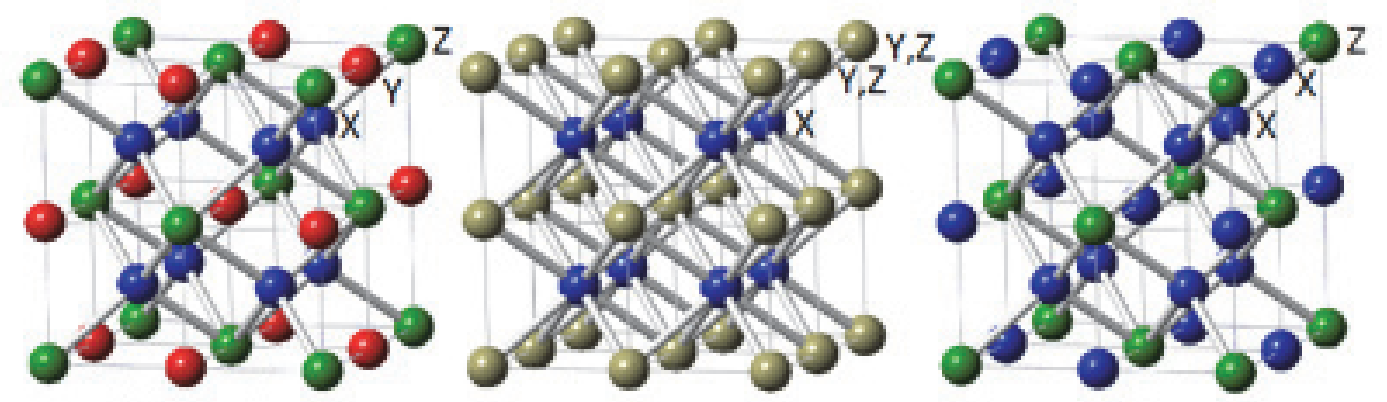

Full Heusler, L2 $2_{1}$

$$
\mathrm{X}_{2} \mathrm{YZ}
$$

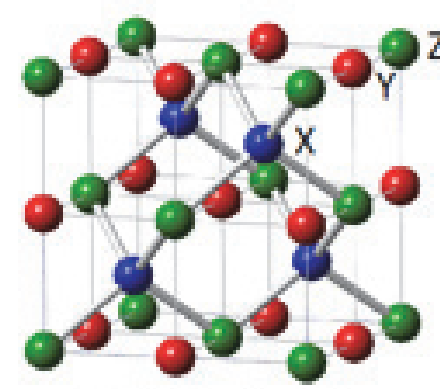

Half Heusler, $\mathrm{C}_{\mathrm{b}}$ $\mathrm{XYZ}$
B2

$X_{2} Y Z$

$\mathrm{DO}_{3}$
$\mathrm{X}_{2} \times \mathrm{Z}$

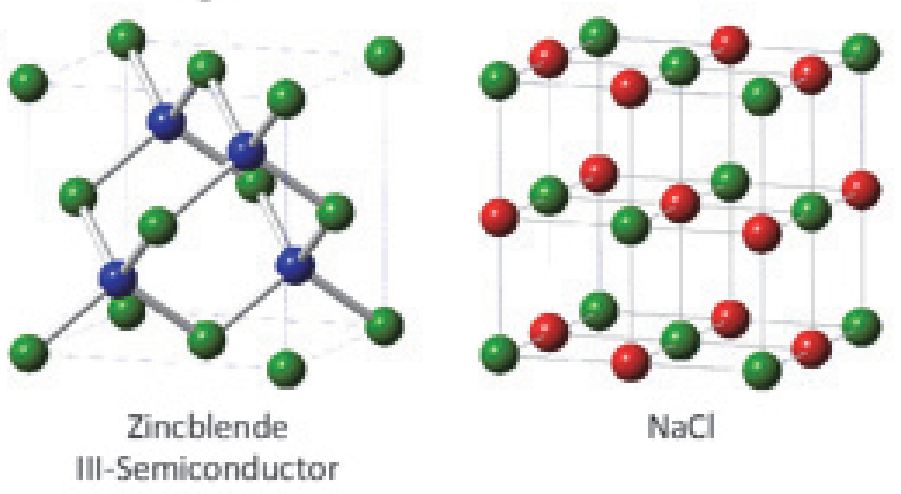

Fig.2 


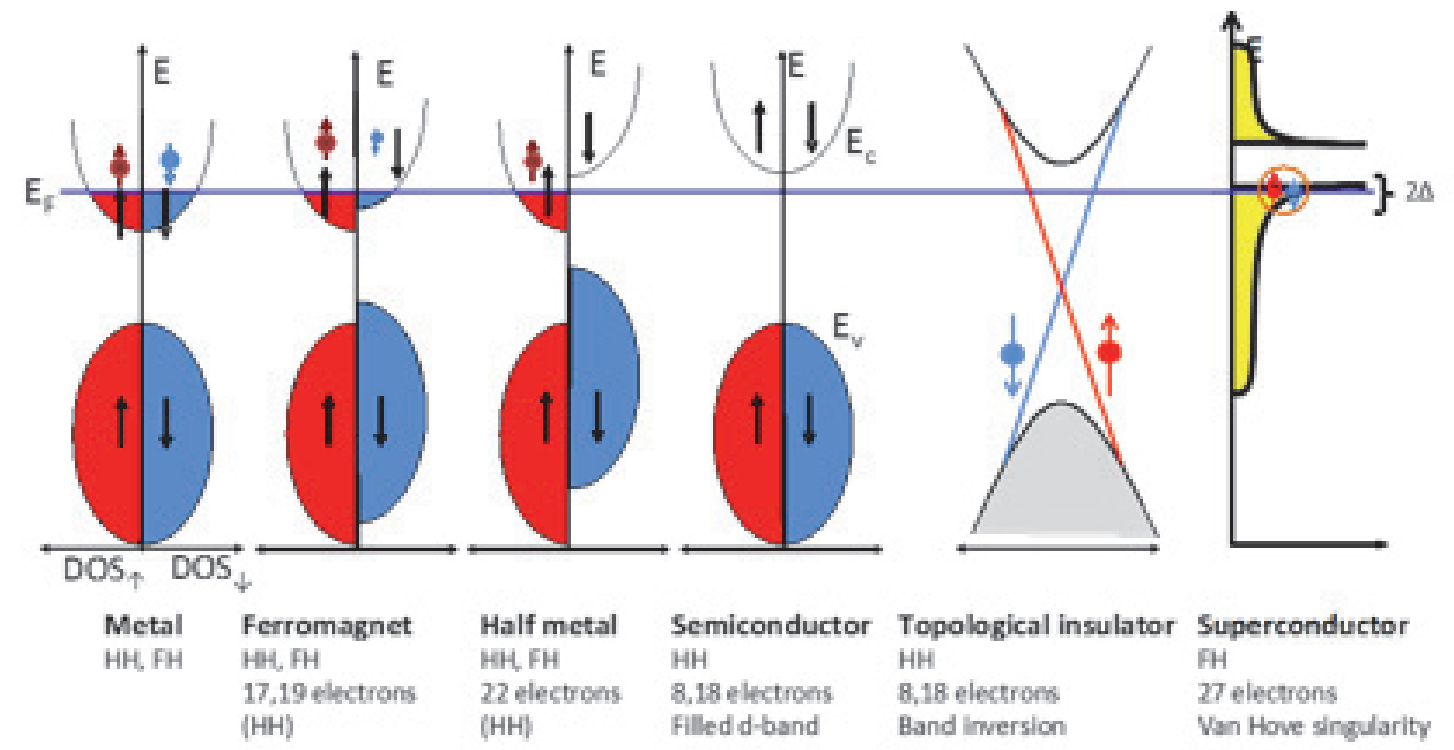

Fig. 3 


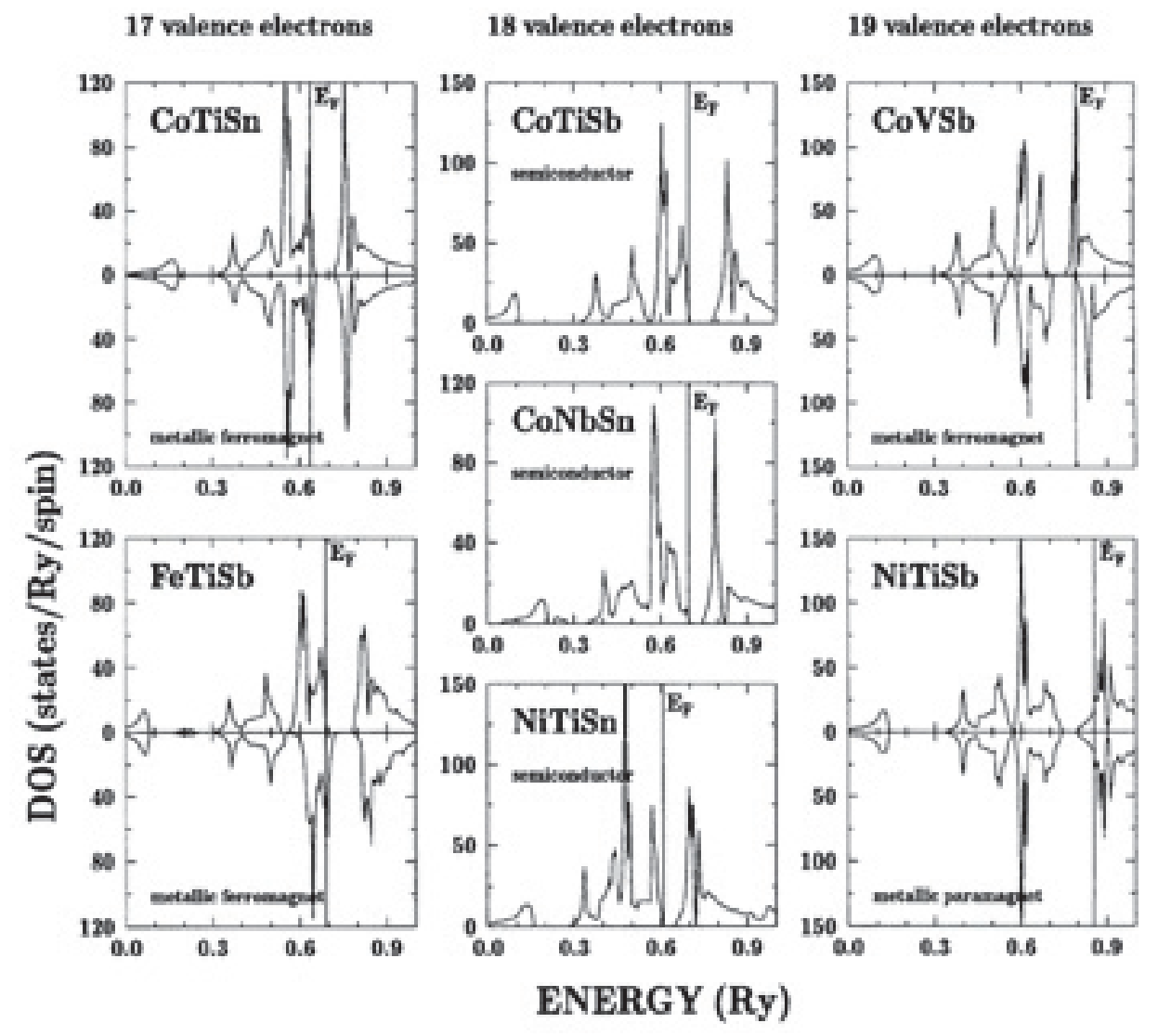

Fig. 4 


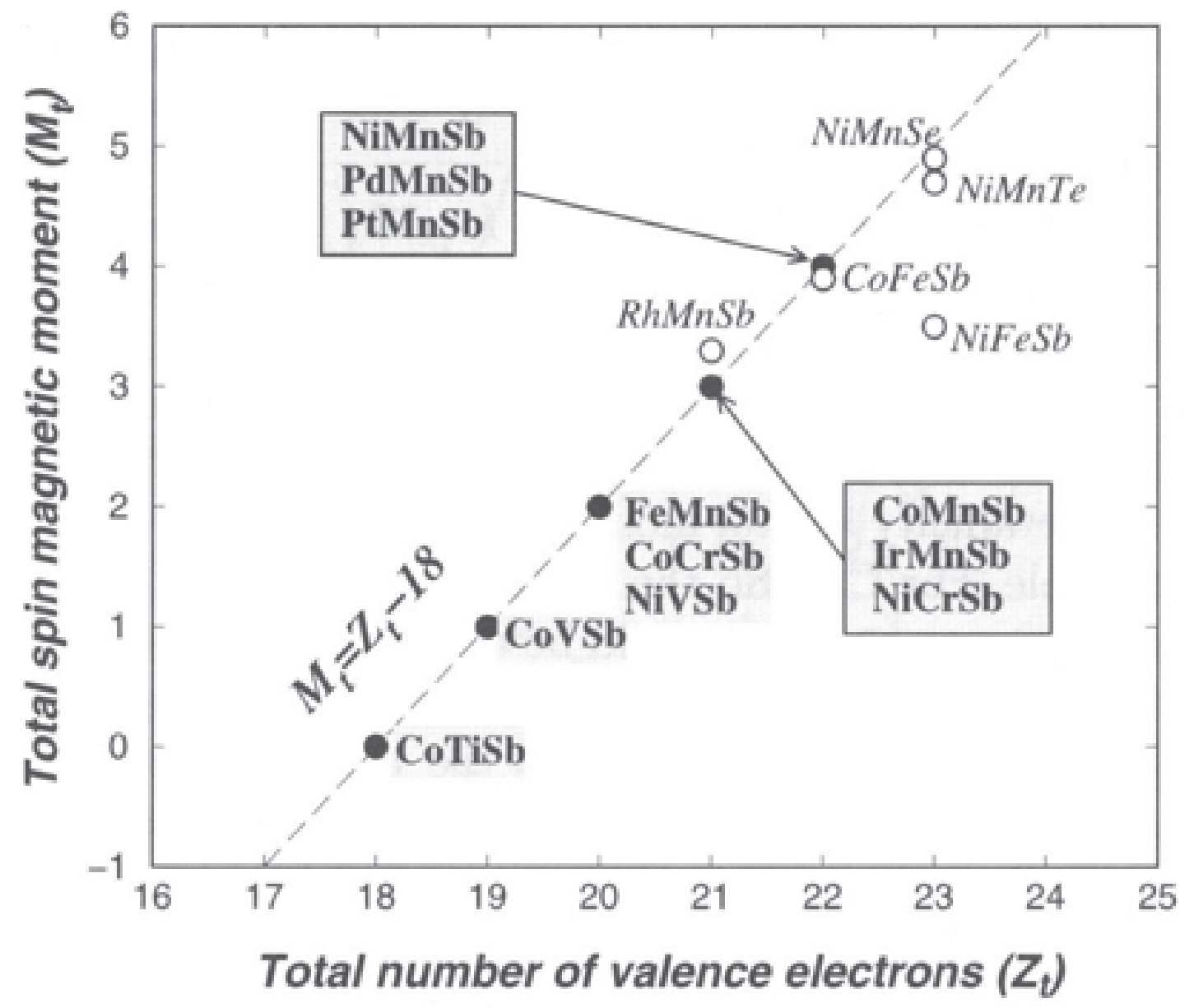

Fig.5 


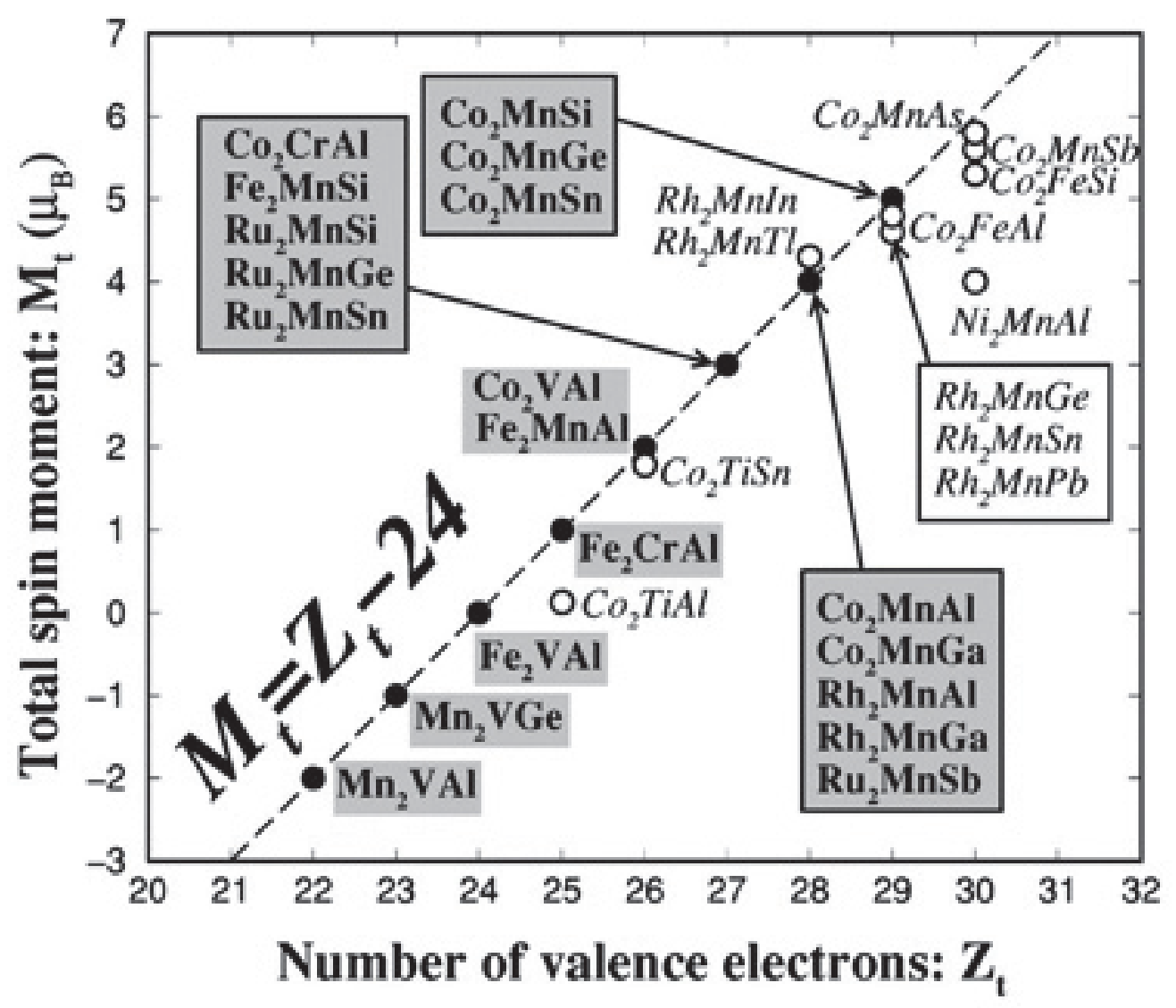

Fig.6 


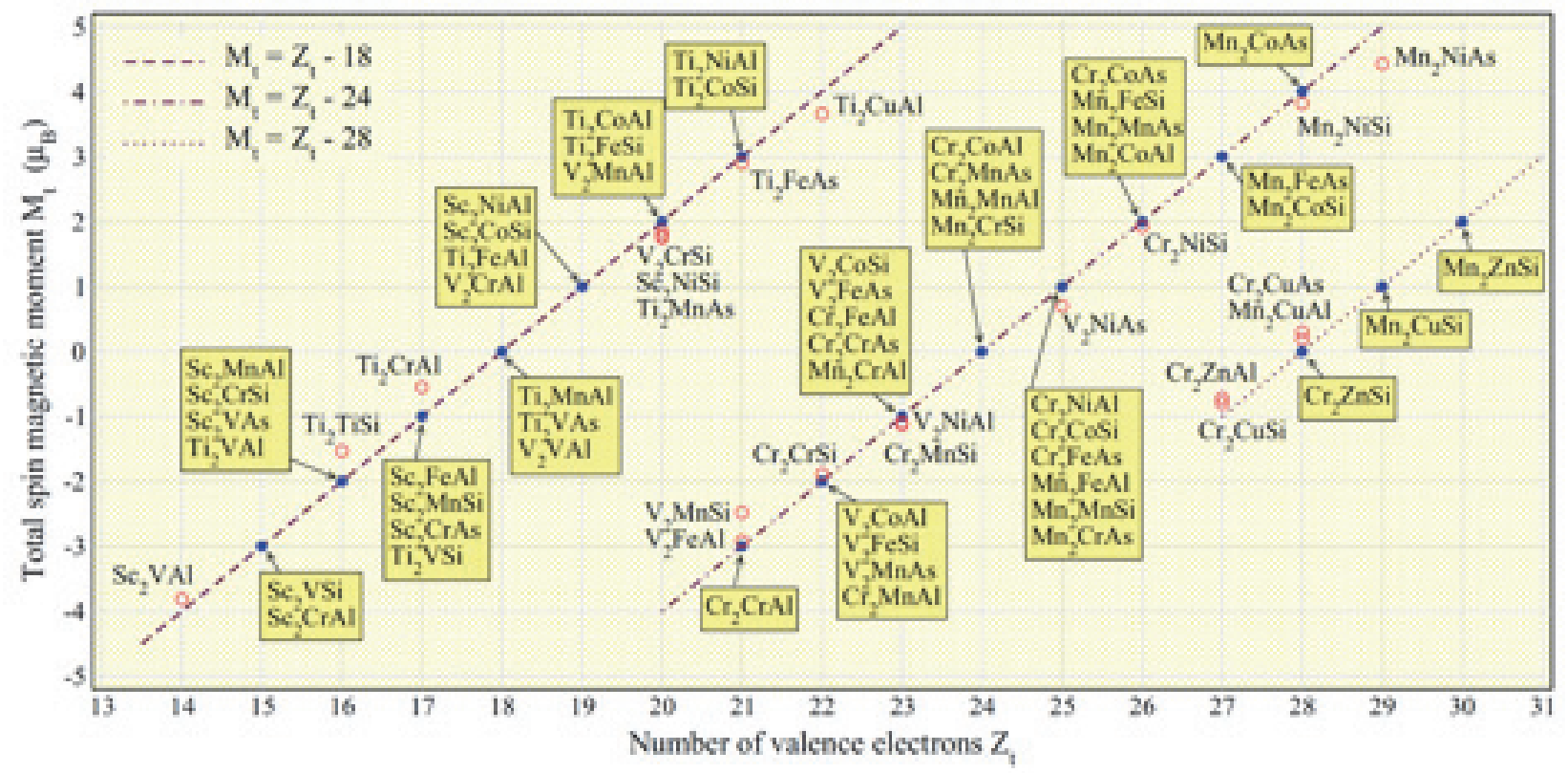

Fig.7 


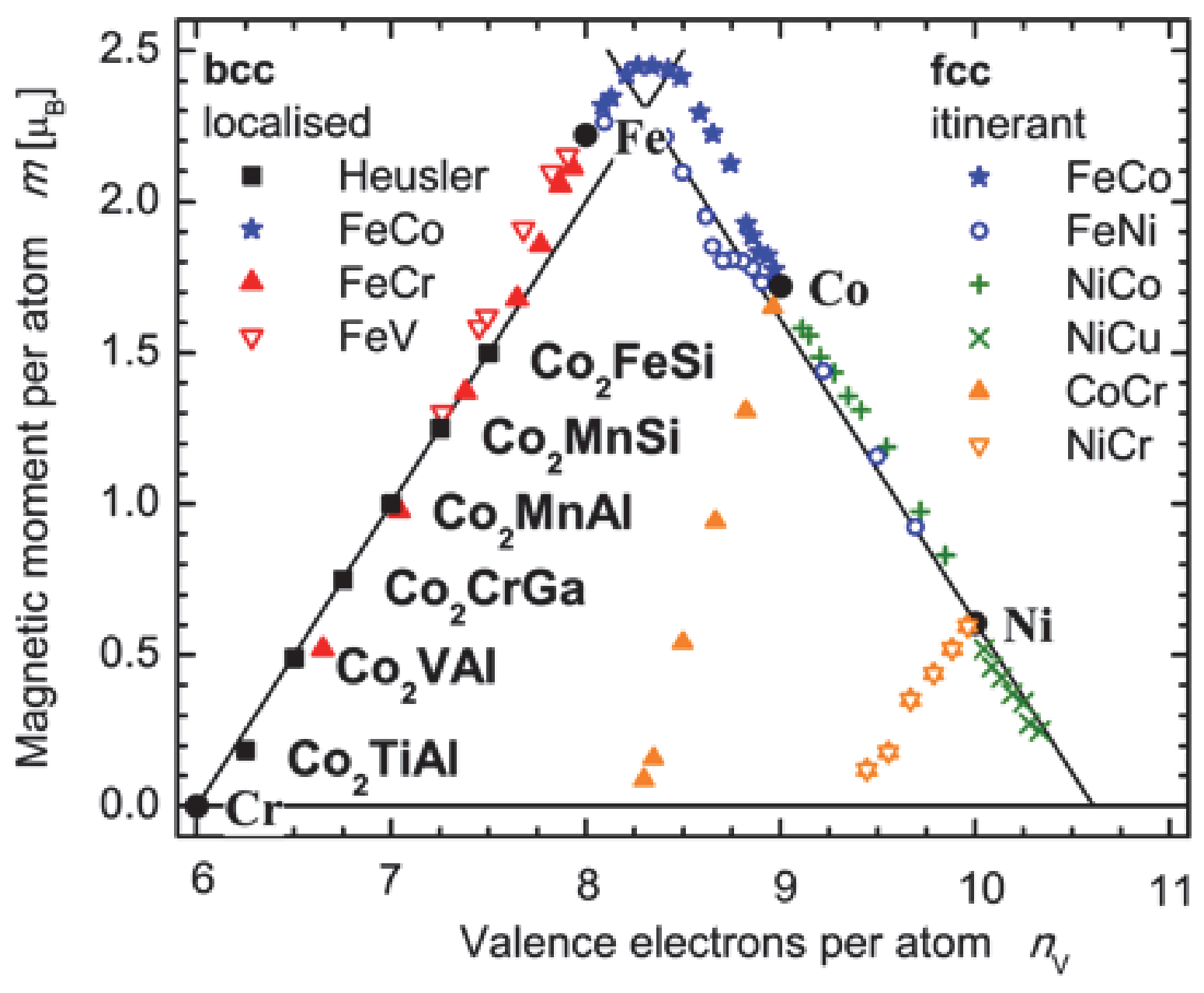

Fig.8 


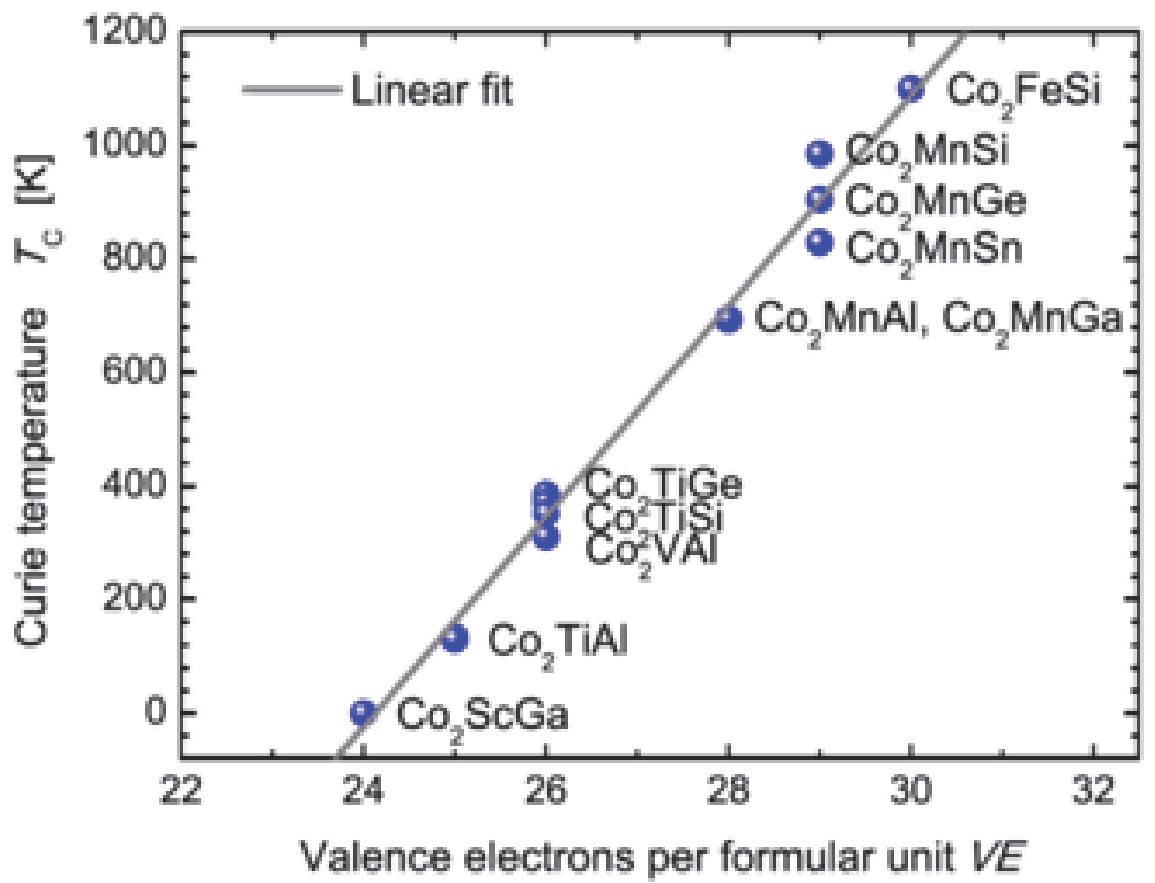

Fig. 9 


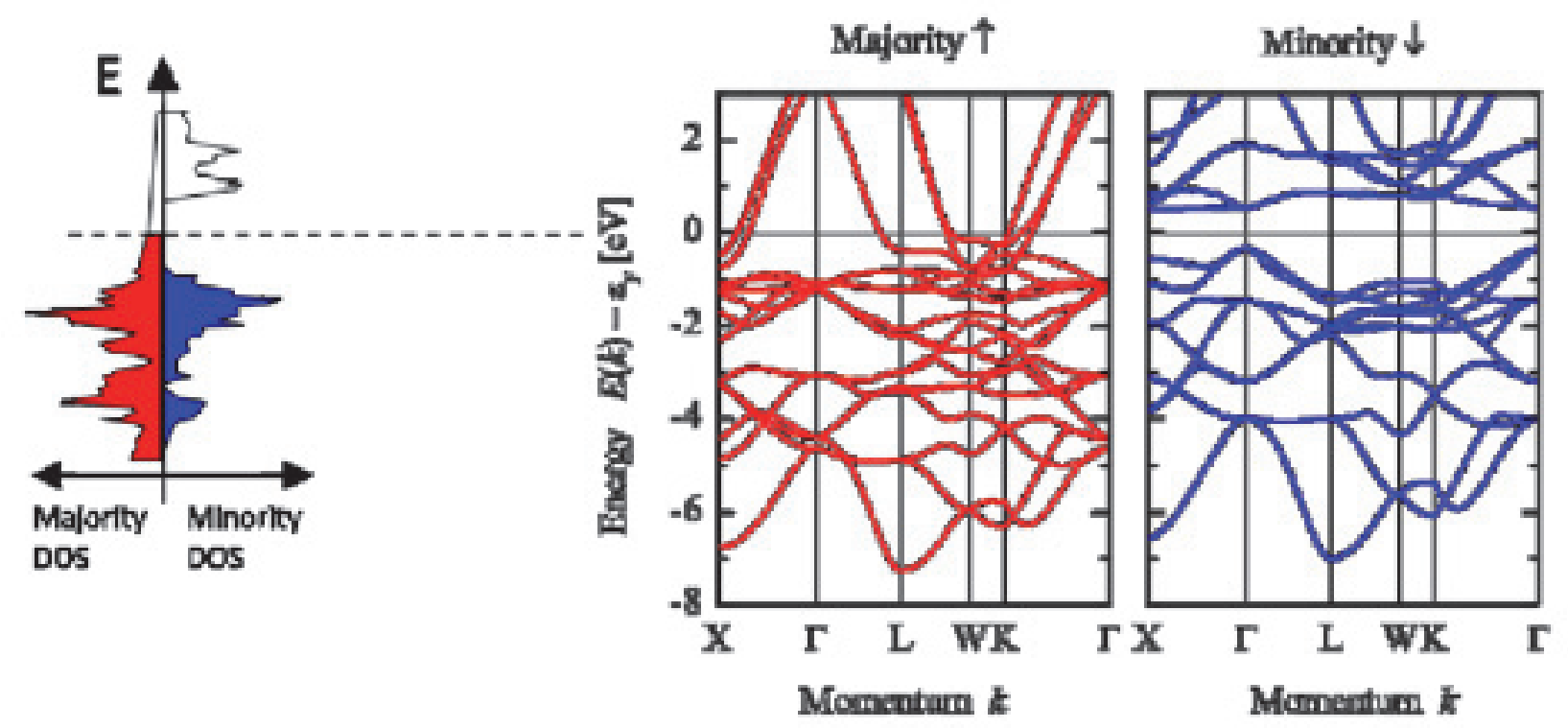

Fig. 10 


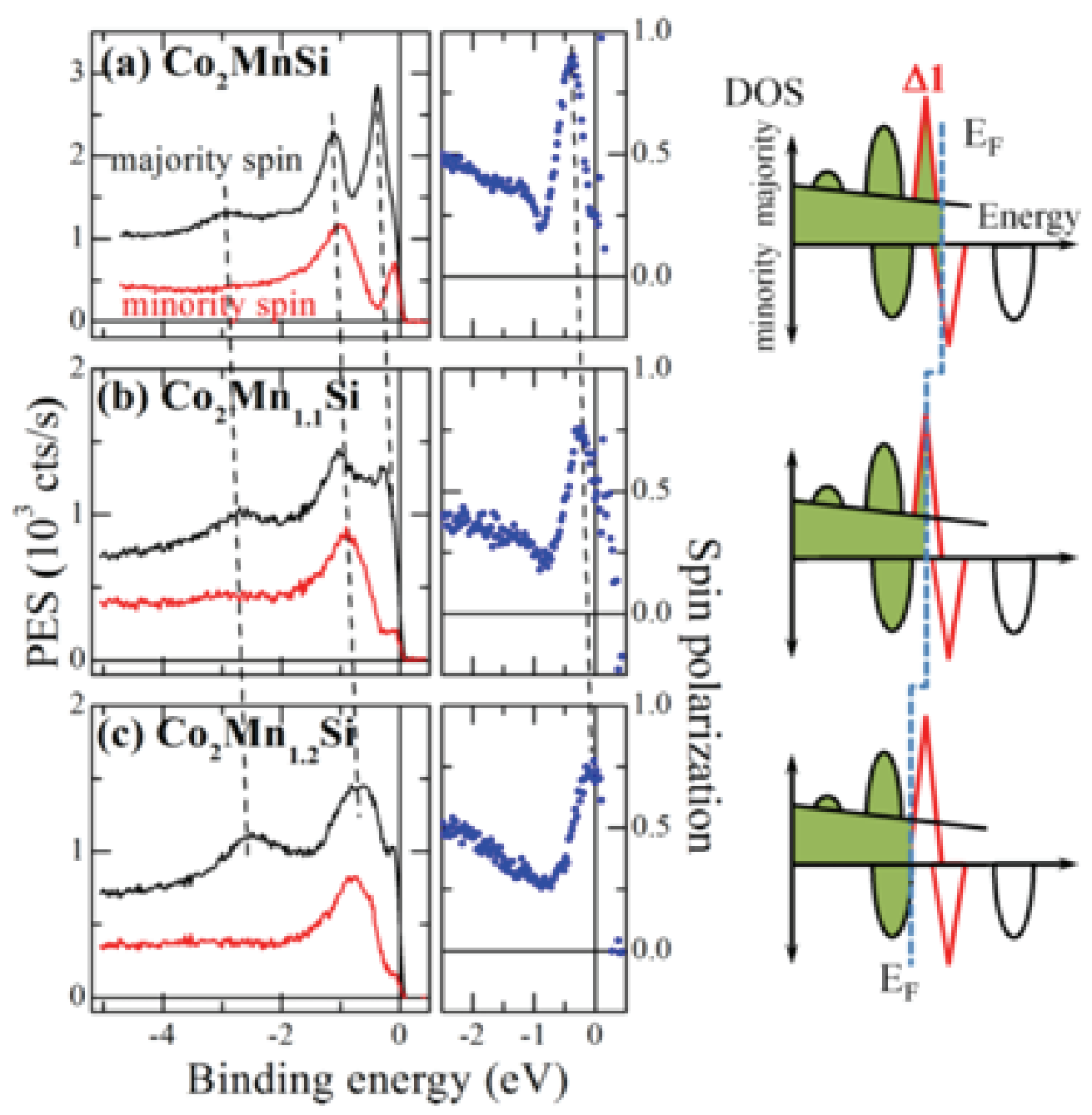

Fig. 11 


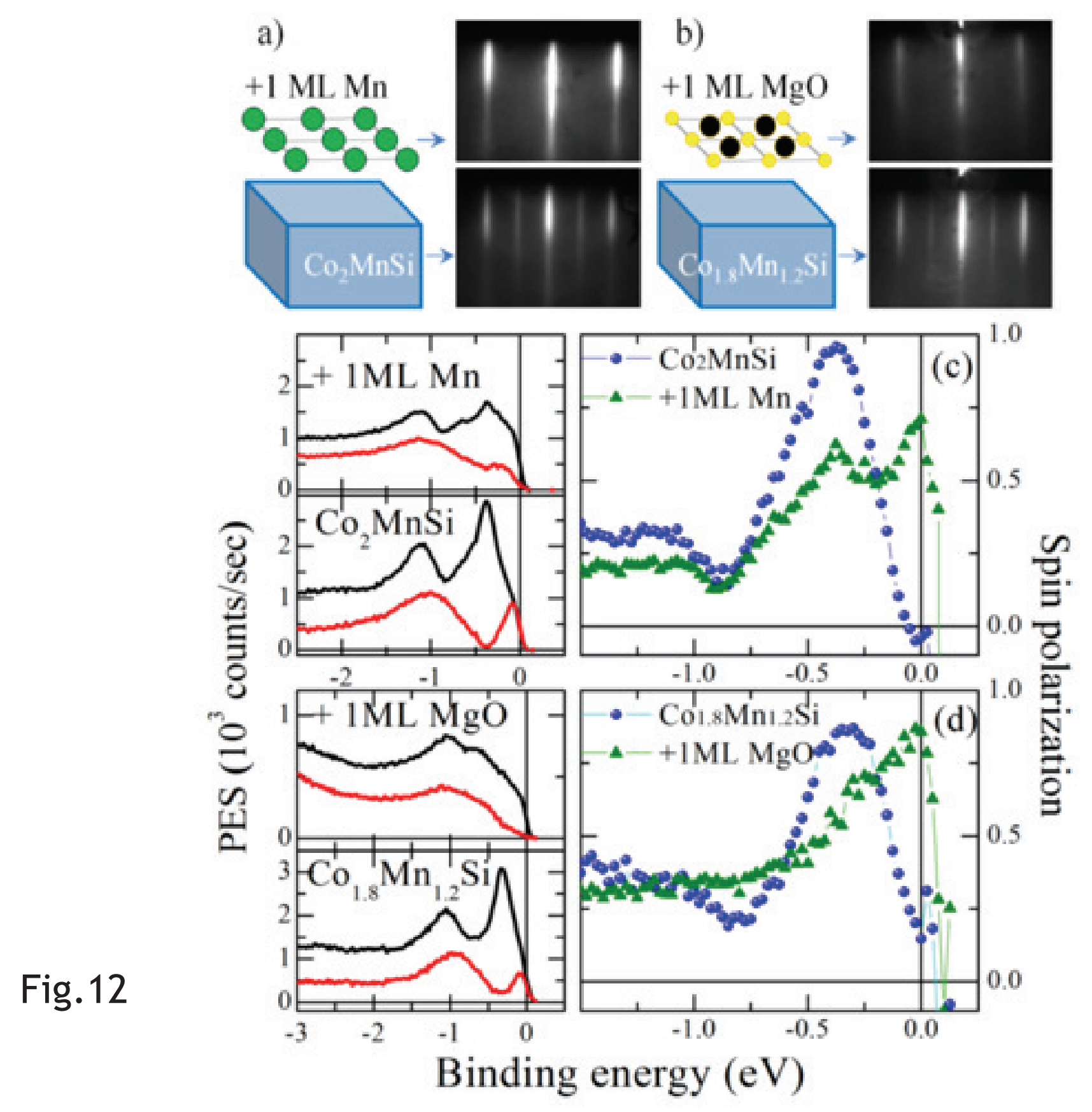




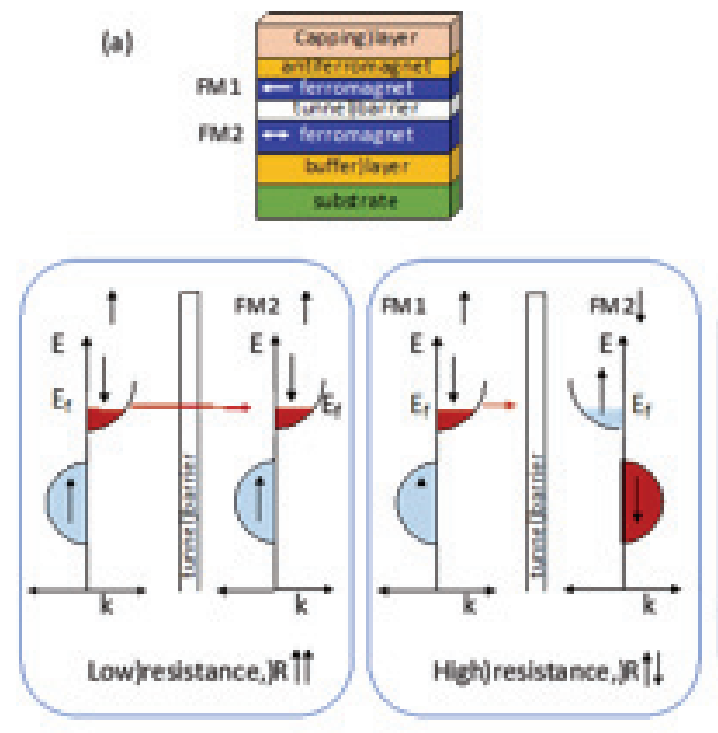

Magretic Tunnel Junction (MT)) (b)
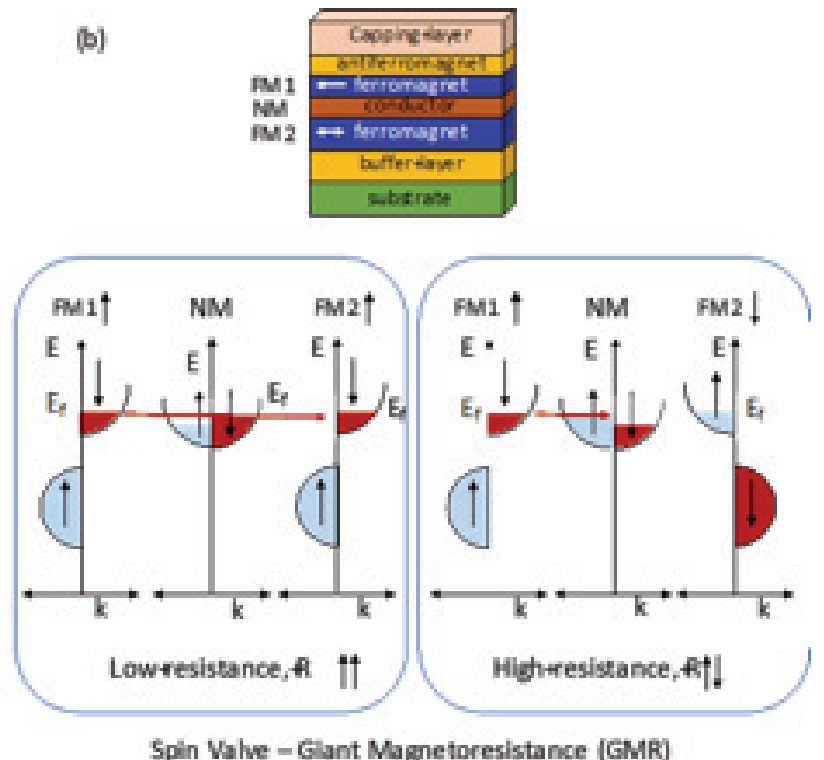

Spin Value - Glant Magretoresistance (GMR)

Fig.13 

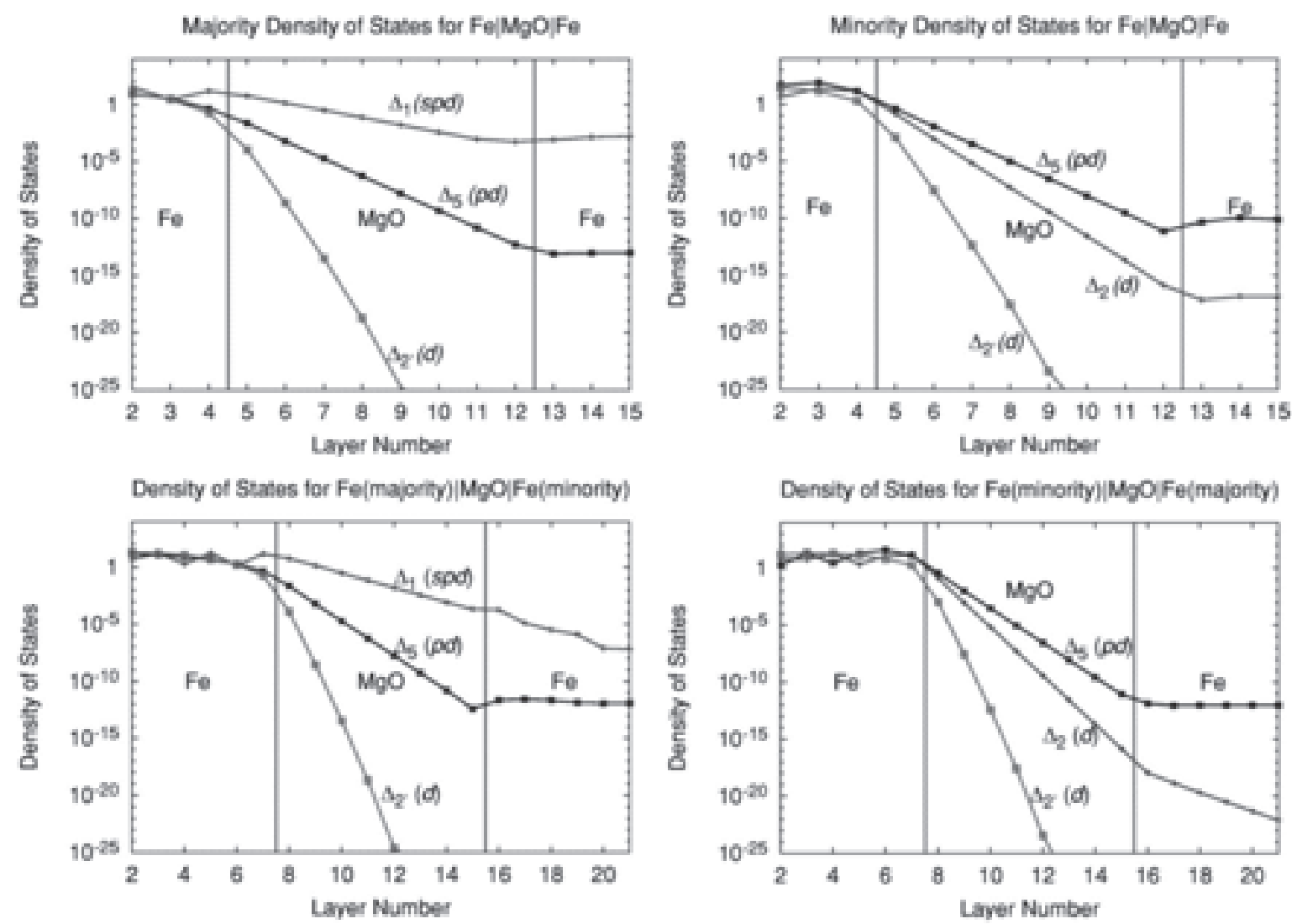

Fig.14 


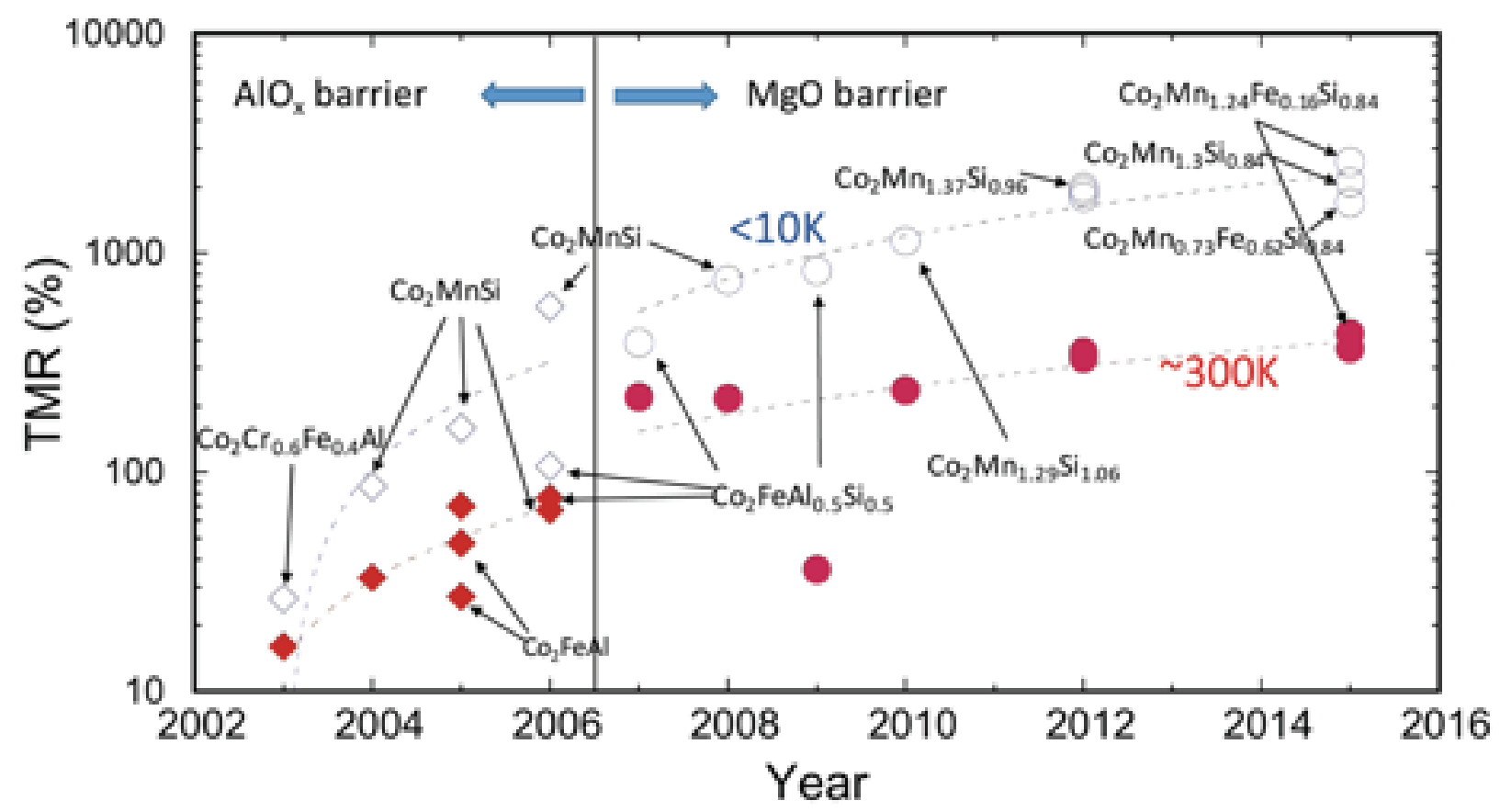

Fig.15 

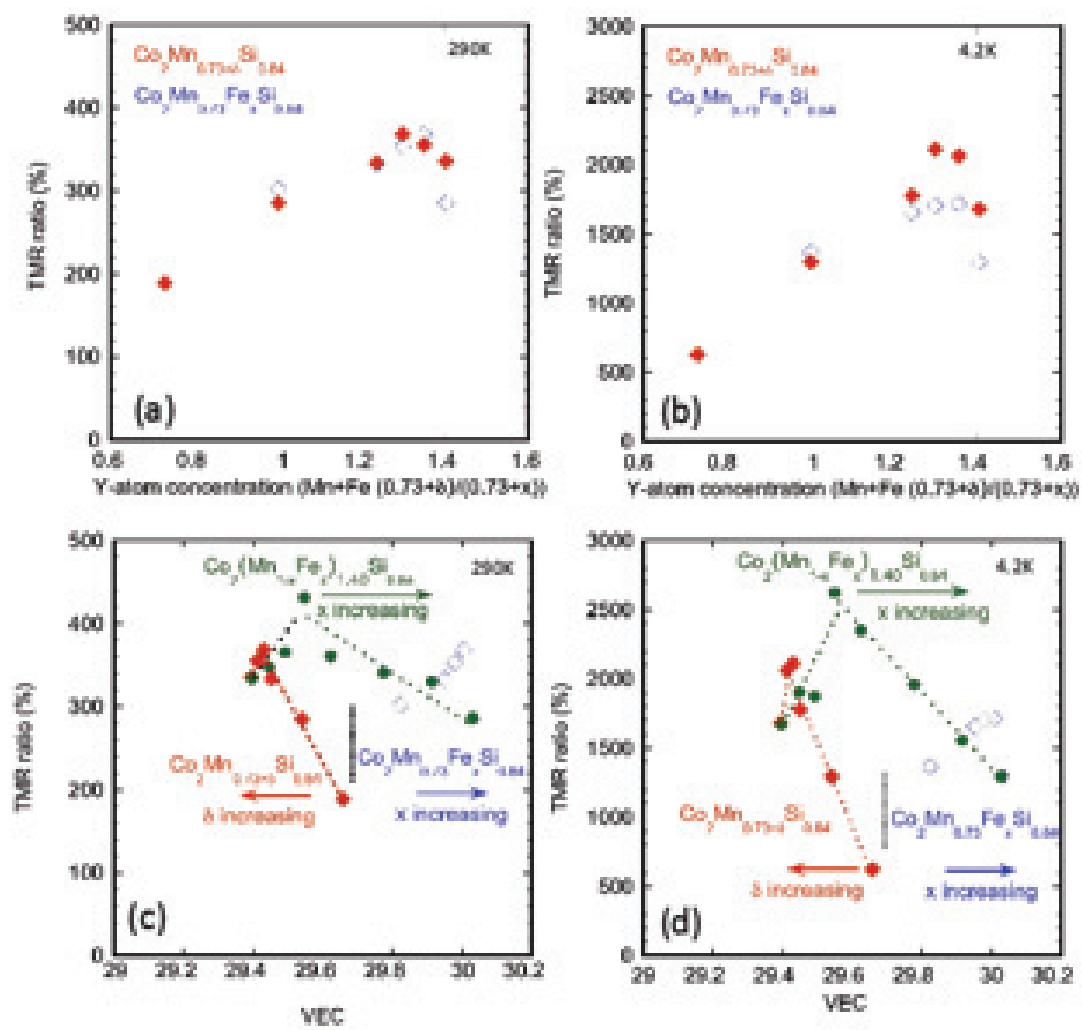

Fig.16 
(a)

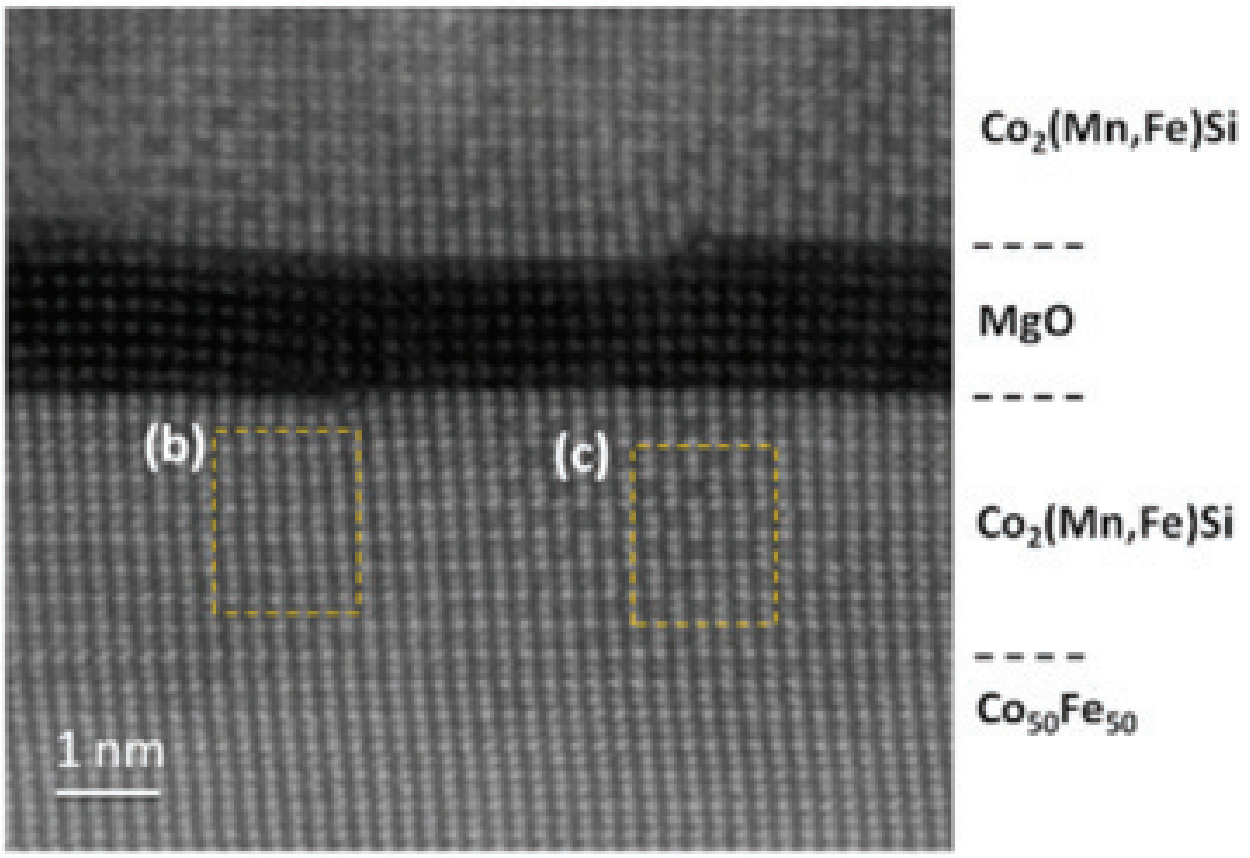

(b)

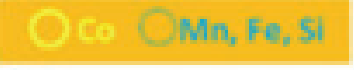

(c)
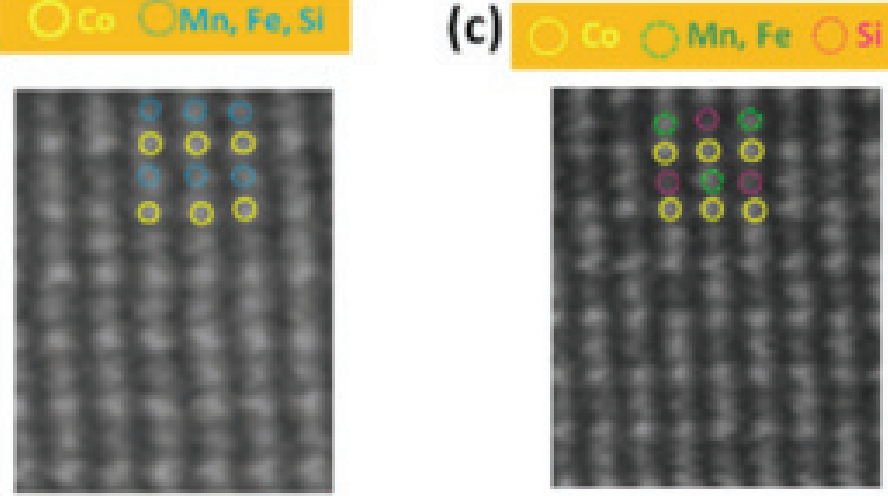

Fig. 17 
Spin manipulation

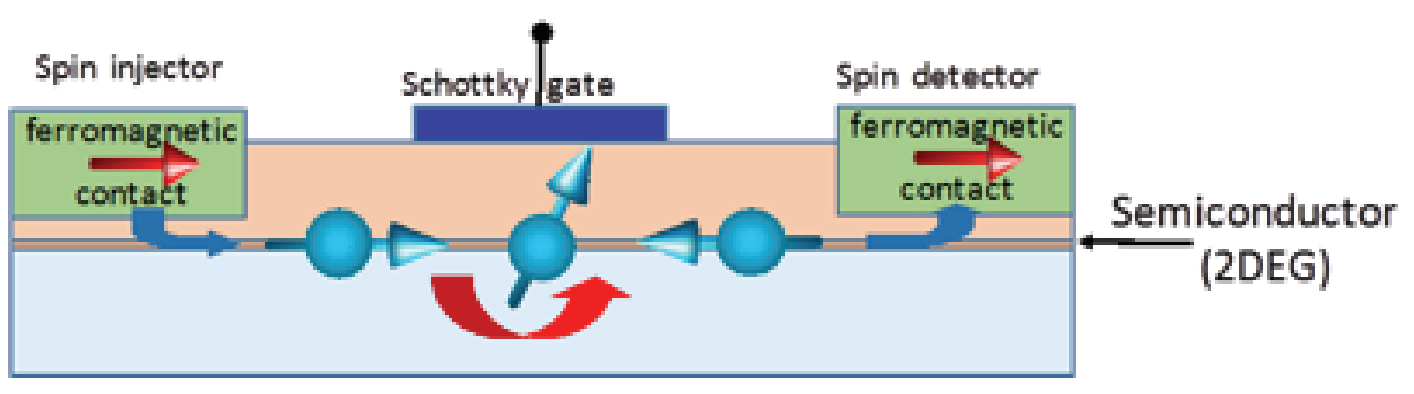

Fig. 18 


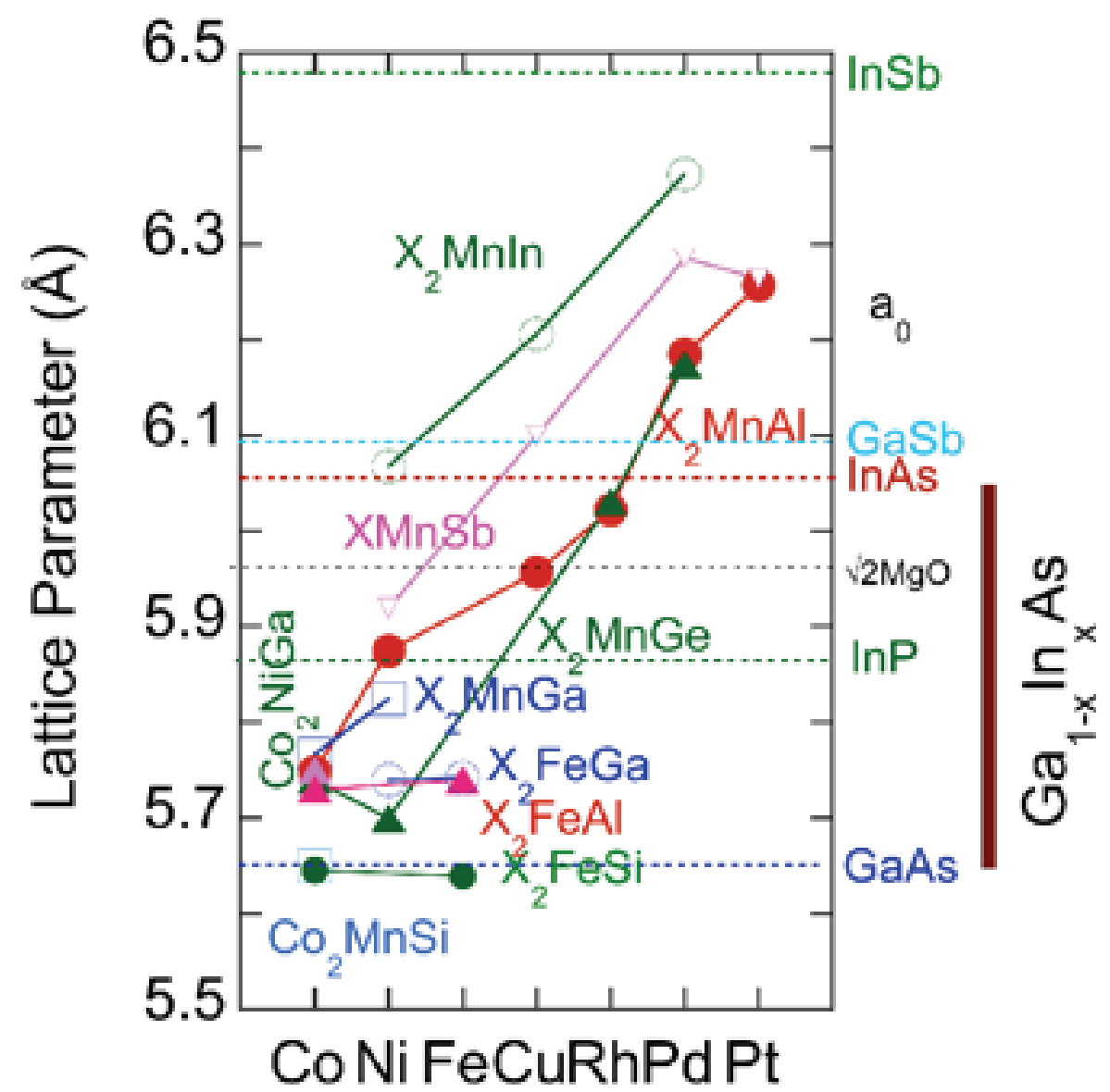

Fig.19 Element (X) 


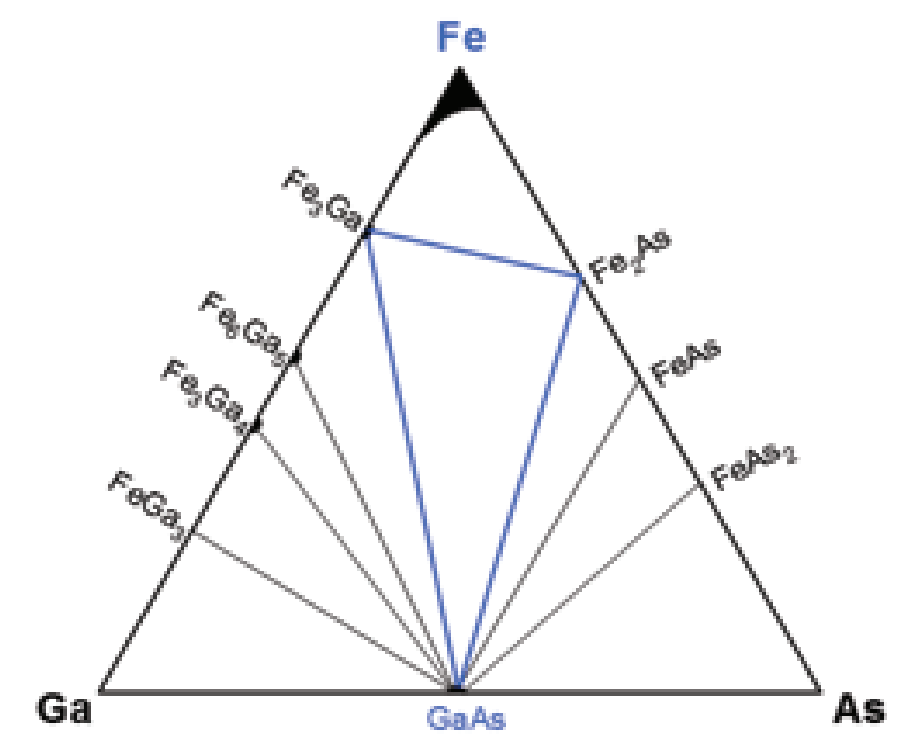

Fig.20 


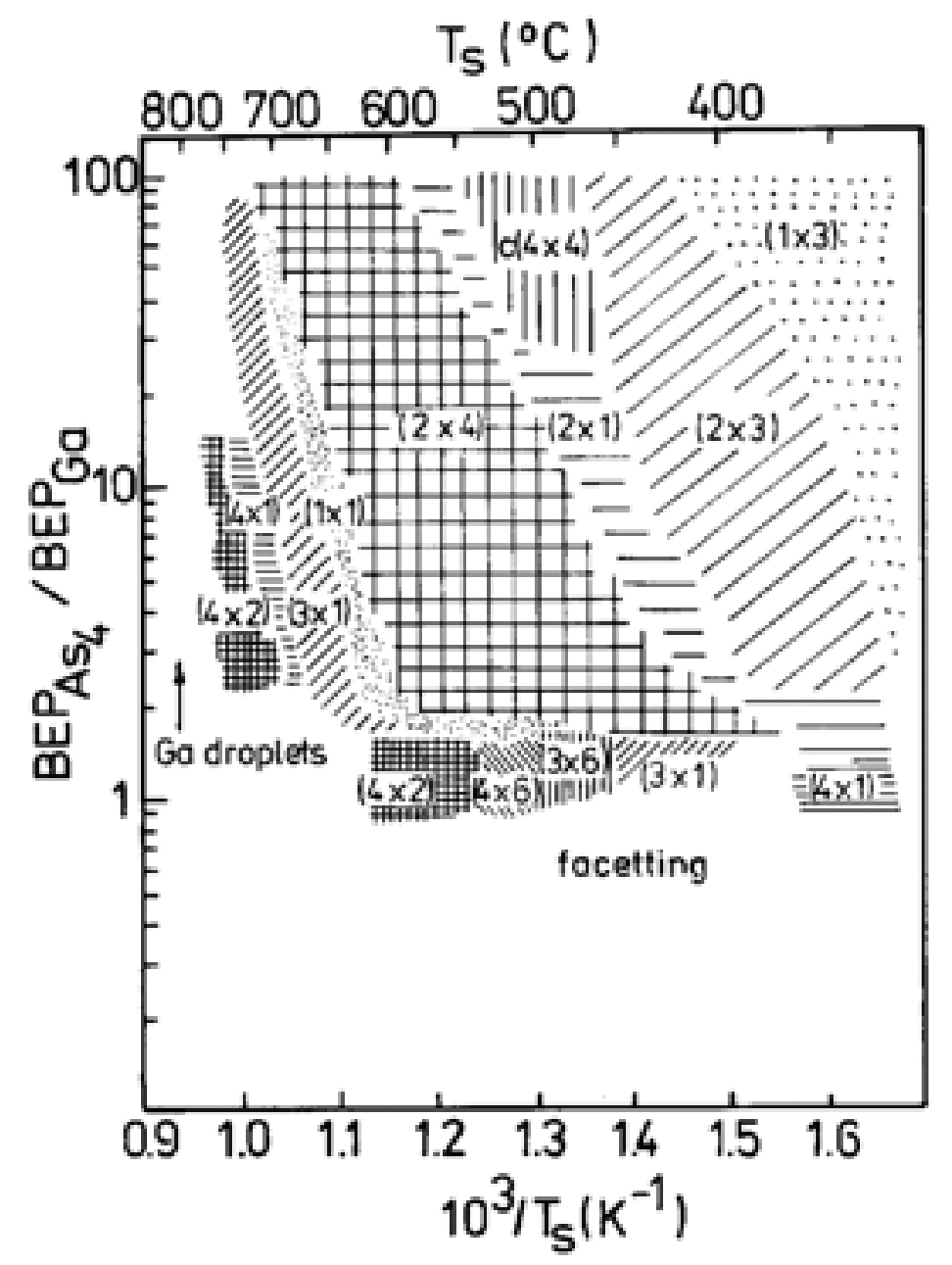

Fig. 21 


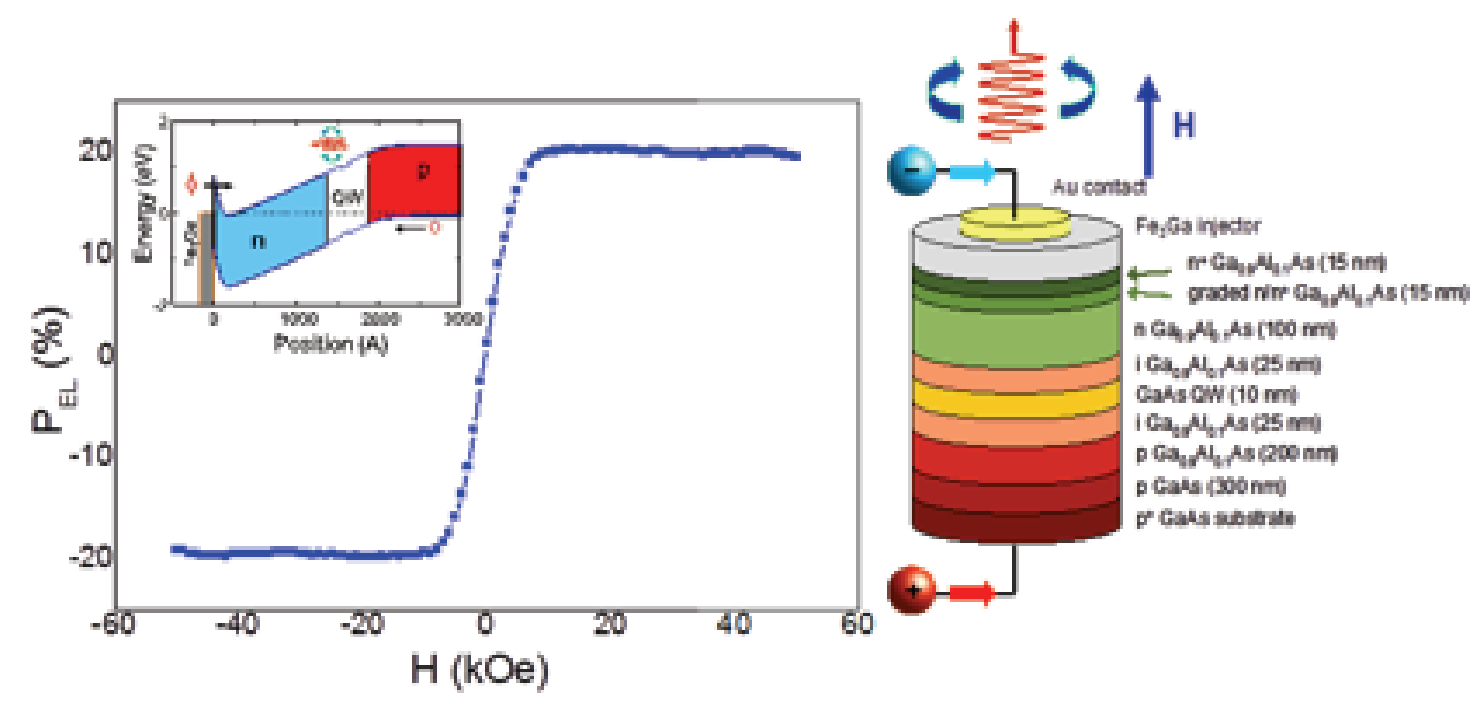

Fig.22 

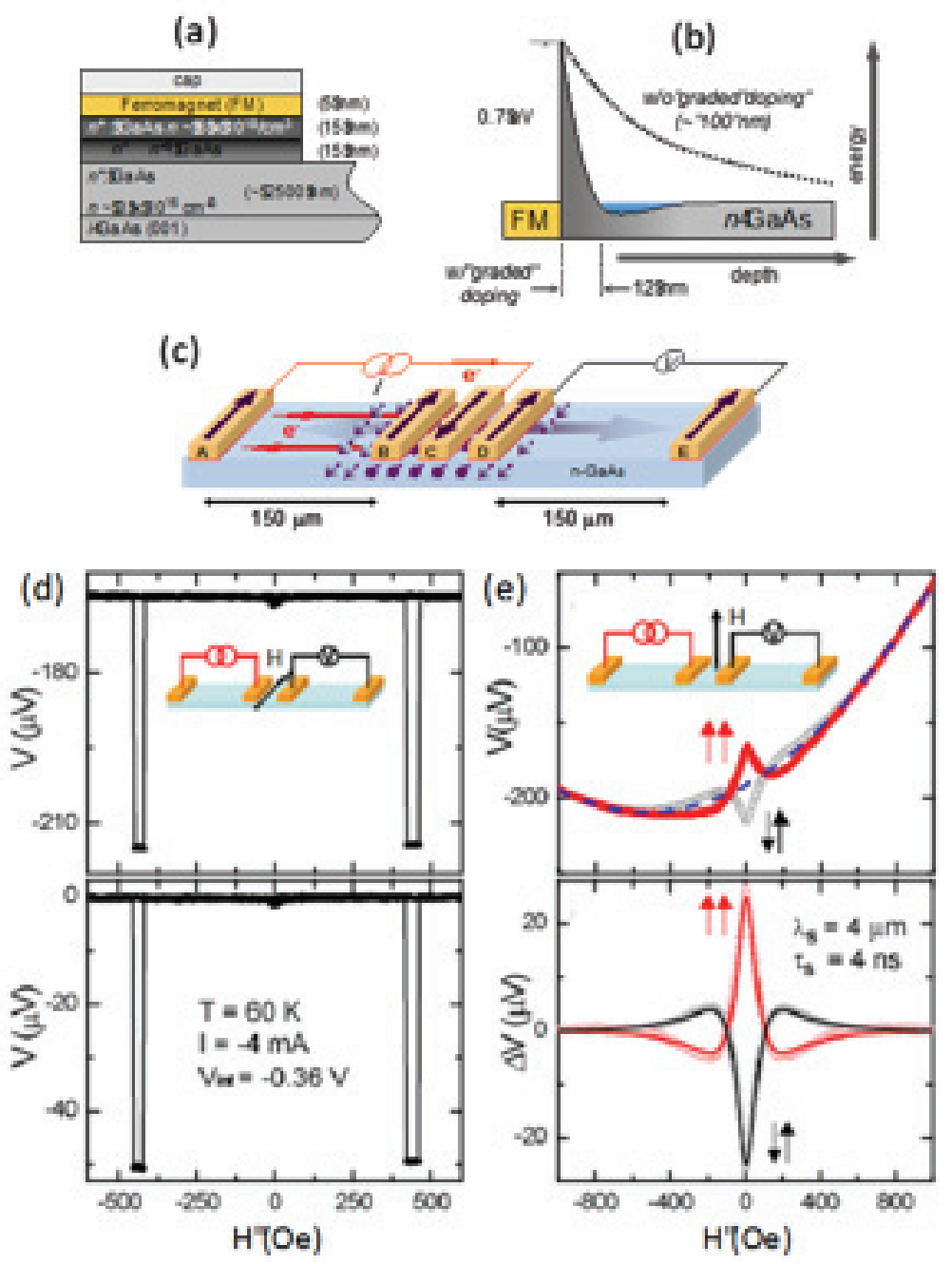

Fig.23 

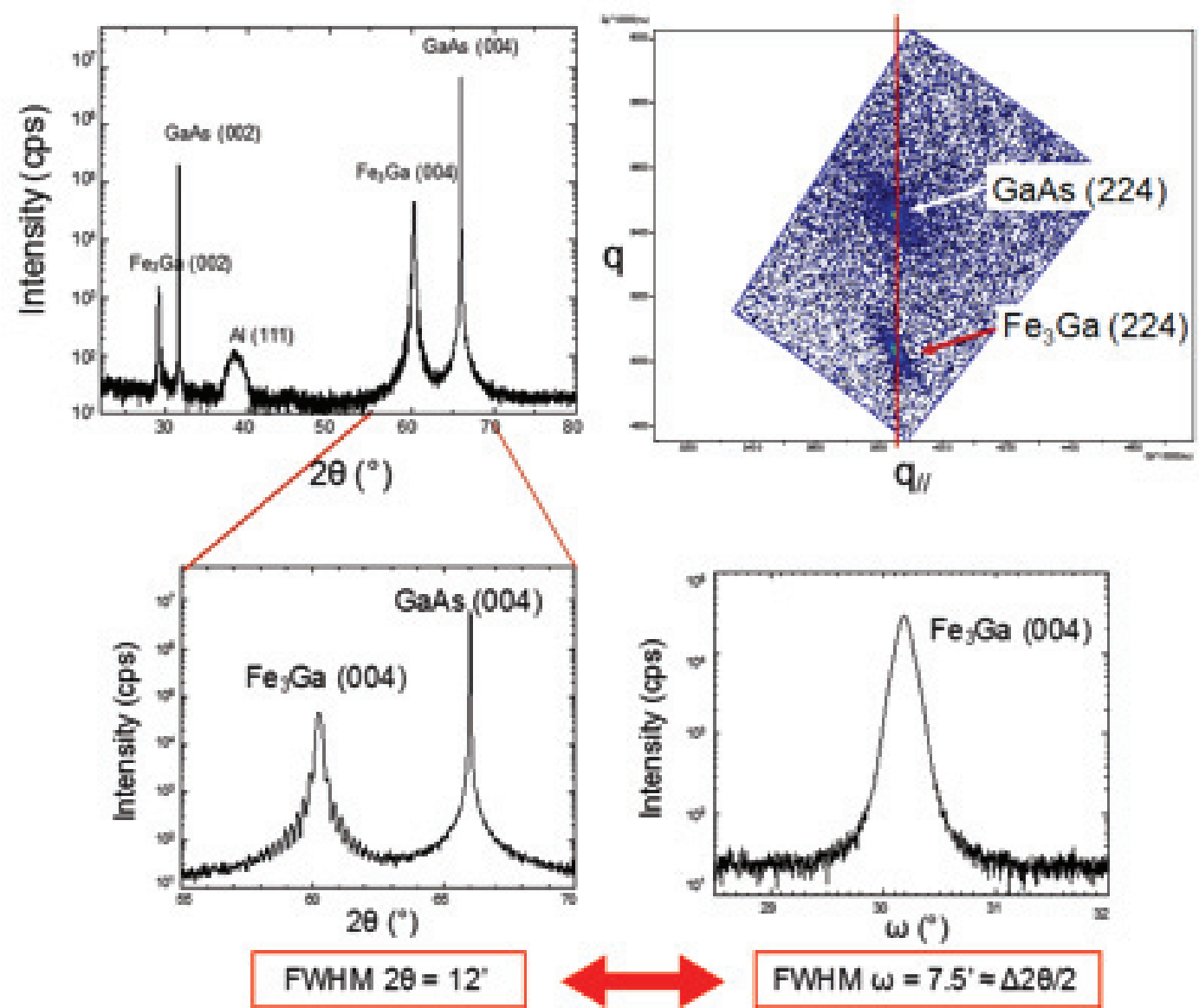

FWHM $\omega=7.5^{\prime}=\Delta 2 \theta / 2$

Fig.24 
- GaAs surface reconstruction

- As-rich

- Ga-rich

- how As- or Ga- rich, multiple reconstructions are possible

- Initiation of $\mathrm{Fe}_{3} \mathrm{Ga}$ growth

- Fe first

- Ga first

- Co-deposition of $\mathrm{Fe}+\mathrm{Ga}$

10000 00000 0000 00000 00000

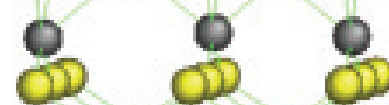

$\mathrm{Ga}$

As

8

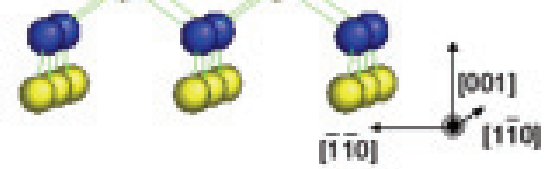

Fig. 25 


\begin{tabular}{|c|c|}
\hline $\begin{array}{l}10 \mathrm{~nm} \mathrm{Al} \mathrm{cap} \\
2.5 \mathrm{~nm} \mathrm{Fe} \mathrm{Ga}_{3}\end{array}$ & \\
\hline $2.5 \mathrm{~nm} \mathrm{LT-Fe} \mathrm{F}_{3} \mathrm{Ga}$ & 3. Co-deposition of $\mathrm{Fe}$ and $\mathrm{Ga}$ at $\mathrm{Ts}=300^{\circ} \mathrm{C}$ \\
\hline $\begin{array}{c}\quad 15 \mathrm{~nm} \\
n^{+} \text {GaAs:Si } \sim 5 \times 10^{18} / \mathrm{cm}^{3}\end{array}$ & \multirow{3}{*}{$\begin{array}{l}\text { 2. Alternative deposition of Fe and } \mathrm{Ga} \text { at } \\
\text { room temperature: The growth initiated either with } \\
\text { (I) } 0.5 \mathrm{ML} \text { Ga or (ii) } 1.5 \mathrm{ML} \text { Fe deposition } \\
\text { Transfer to metal deposition }\end{array}$} \\
\hline $\begin{array}{c}15 \mathrm{~nm} \\
n^{-\cdots>n^{+} \mathrm{GaAs}: S i}\end{array}$ & \\
\hline$\underset{n-\mathrm{GaAs}: \mathrm{mi}}{2.5 \mathrm{~m}}$ & \\
\hline 500nm I GaAs buffer & \multirow{2}{*}{$\begin{array}{l}\text { 1. Annealing after the growth to obtain different } \\
\text { GaAs surface termination: } \\
\text { (i) } \mathrm{T}_{\mathrm{A}}=365^{\circ} \mathrm{C} \text { à As rich }(2 \times 4) / \mathrm{c}(2 \times 8) \text { or } \\
\text { (ii) } \mathrm{TA}_{\mathrm{A}}=500^{\circ} \mathrm{C} \text { à Ga rich }(4 \times 6) / \mathrm{c}(8 \times 2)\end{array}$} \\
\hline SI GaAs substrate (001) & \\
\hline
\end{tabular}

\begin{tabular}{|c|c|c|c|c|c|}
\hline Sample & Interface & Starting surface & $\begin{array}{c}\mathrm{Fe}_{\mathrm{G}} \mathrm{Ga} \\
\text { initiation }\end{array}$ & Ts & \\
\hline A & FeGa-Ga interface & $(4 \times 6) / d(8 \times 2)$ & $\mathrm{Ga}$ & $300^{\circ} \mathrm{C}$ & Ga-rich \\
\hline $\mathrm{B}$ & Fe-Ga interface & $(4 \times 6) / c(8 \times 2)$ & $\mathrm{Fe}$ & $300^{\circ} \mathrm{C}$ & \\
\hline C & FeGa-As interface & $(2 \times 4) / d(2 \times 8)$ & $\mathrm{Ga}$ & $300^{\circ} \mathrm{C}$ & \\
\hline $\mathrm{D}$ & Fe-As interface & $2 \times 4) / c(2 \times 8)$ & $\mathrm{Fe}$ & $300^{\circ} \mathrm{C}$ & \\
\hline$E$ & Fe-As interface & $2 \times 4) / c(2 \times 8)$ & $\mathrm{Fe}$ & $175^{\circ} \mathrm{C}$ & Asrich \\
\hline
\end{tabular}

Fig. 26 


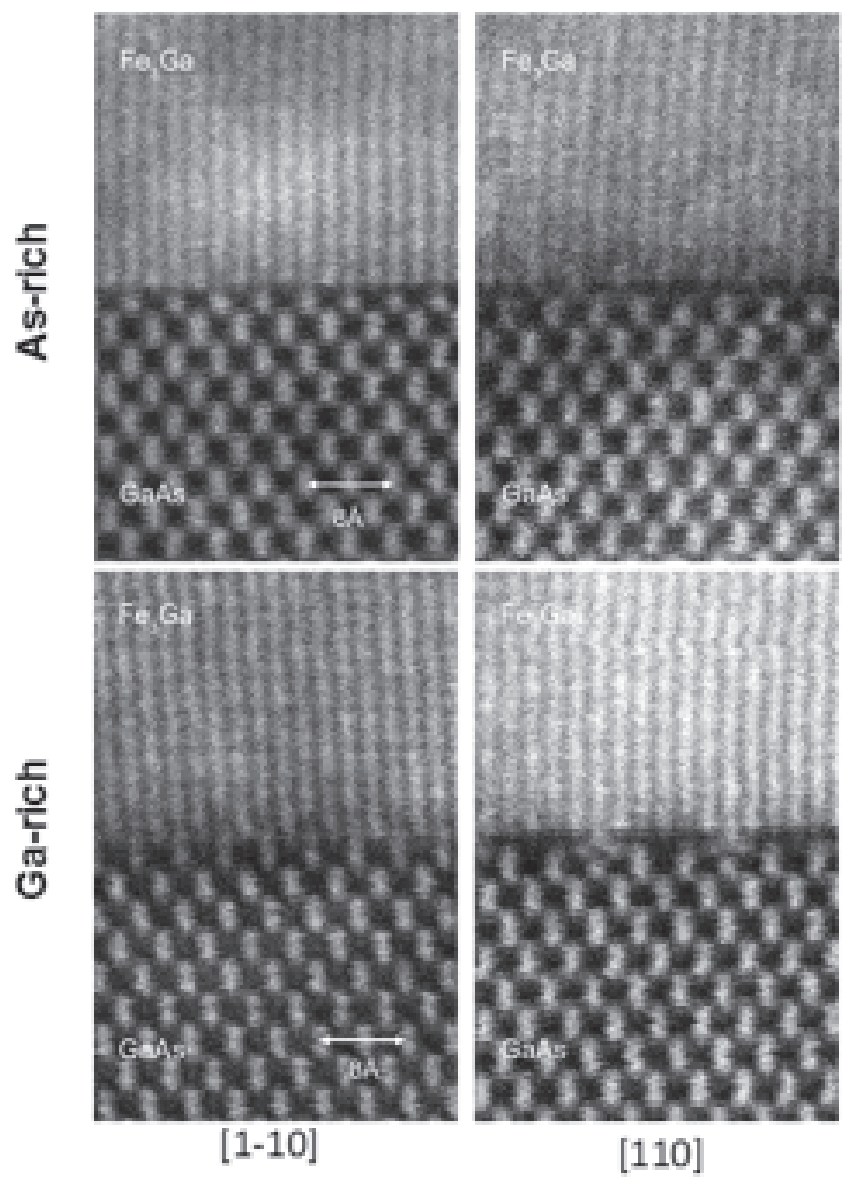

Fig. 27 


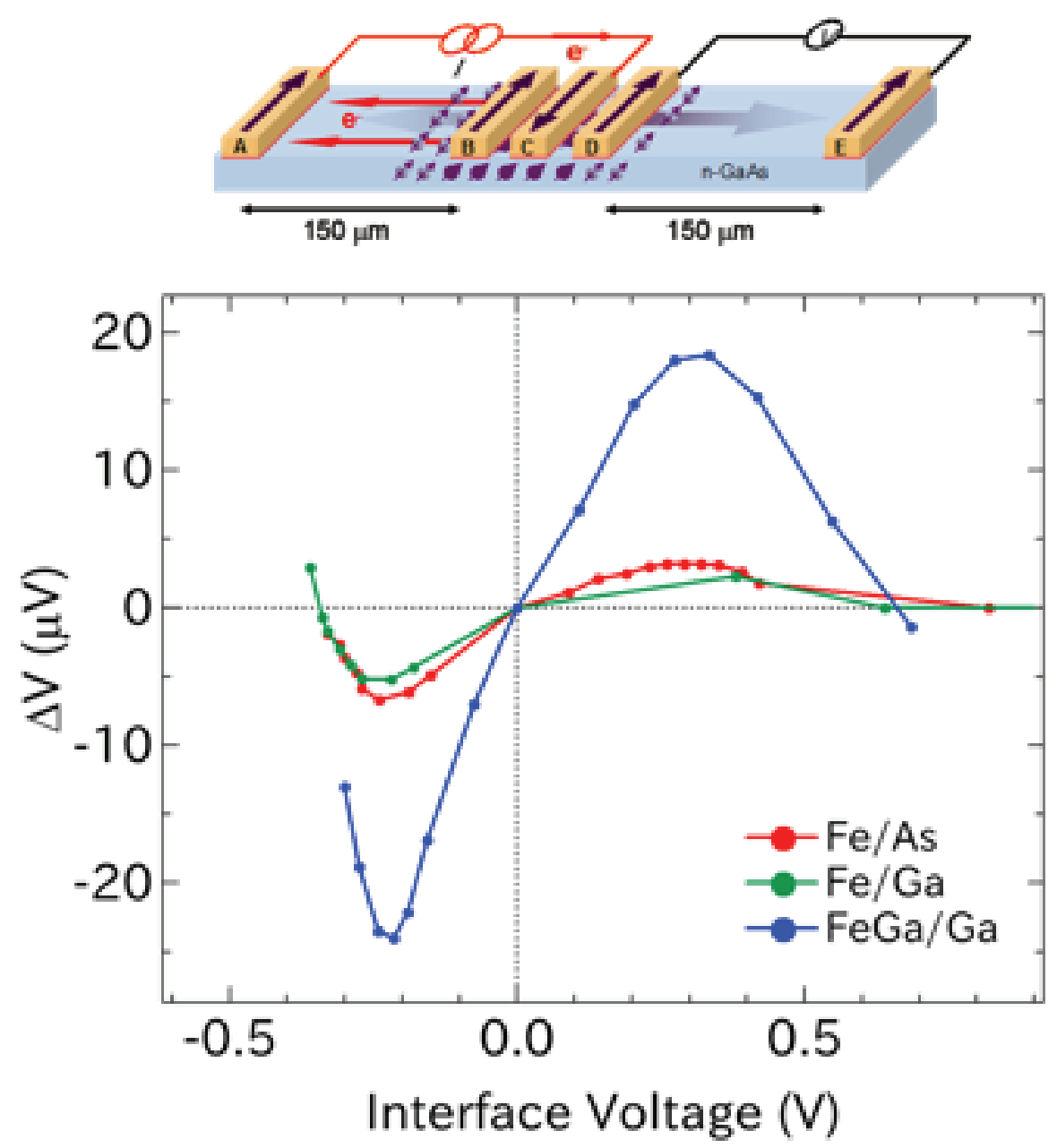

Fig. 28 

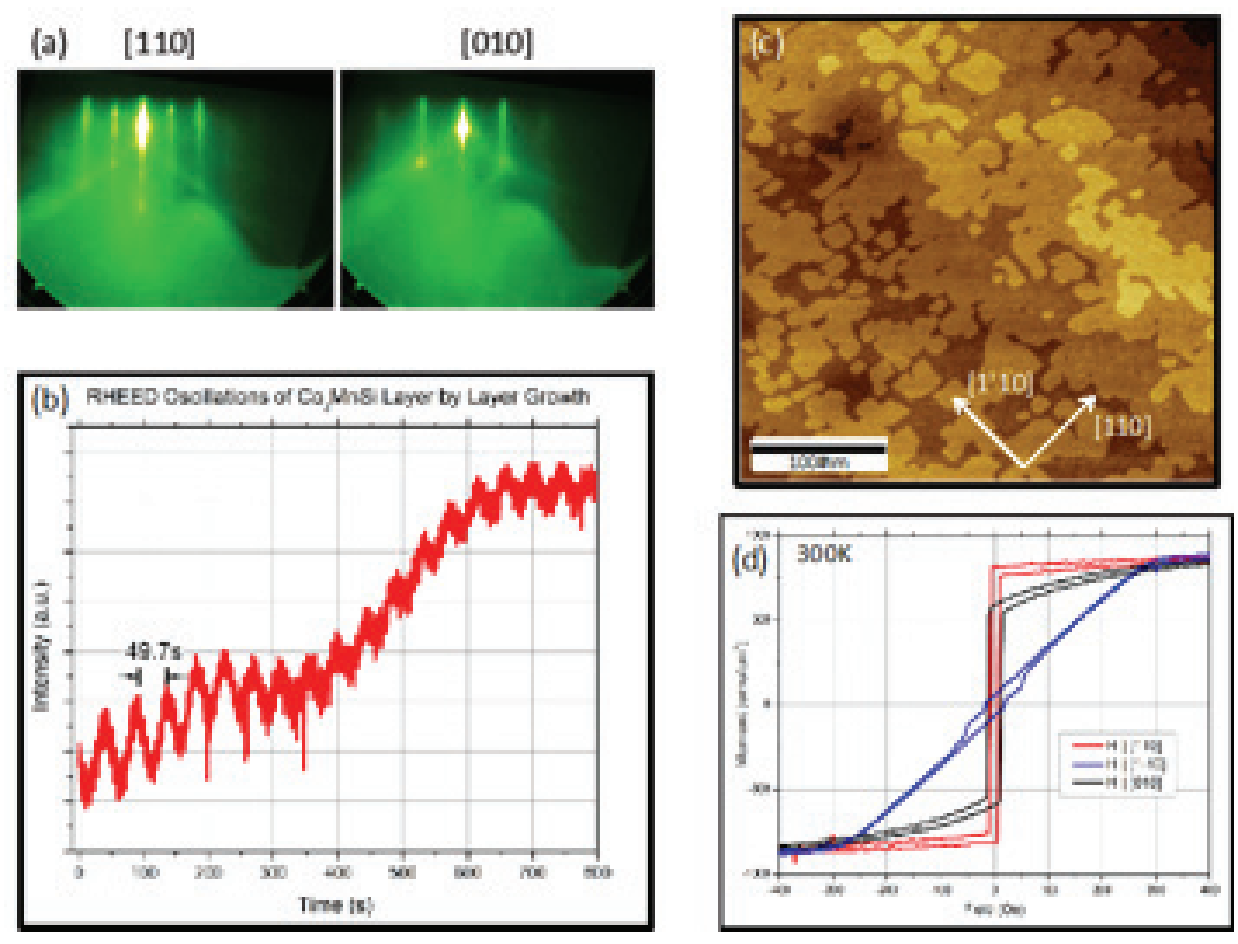

Fig.29 


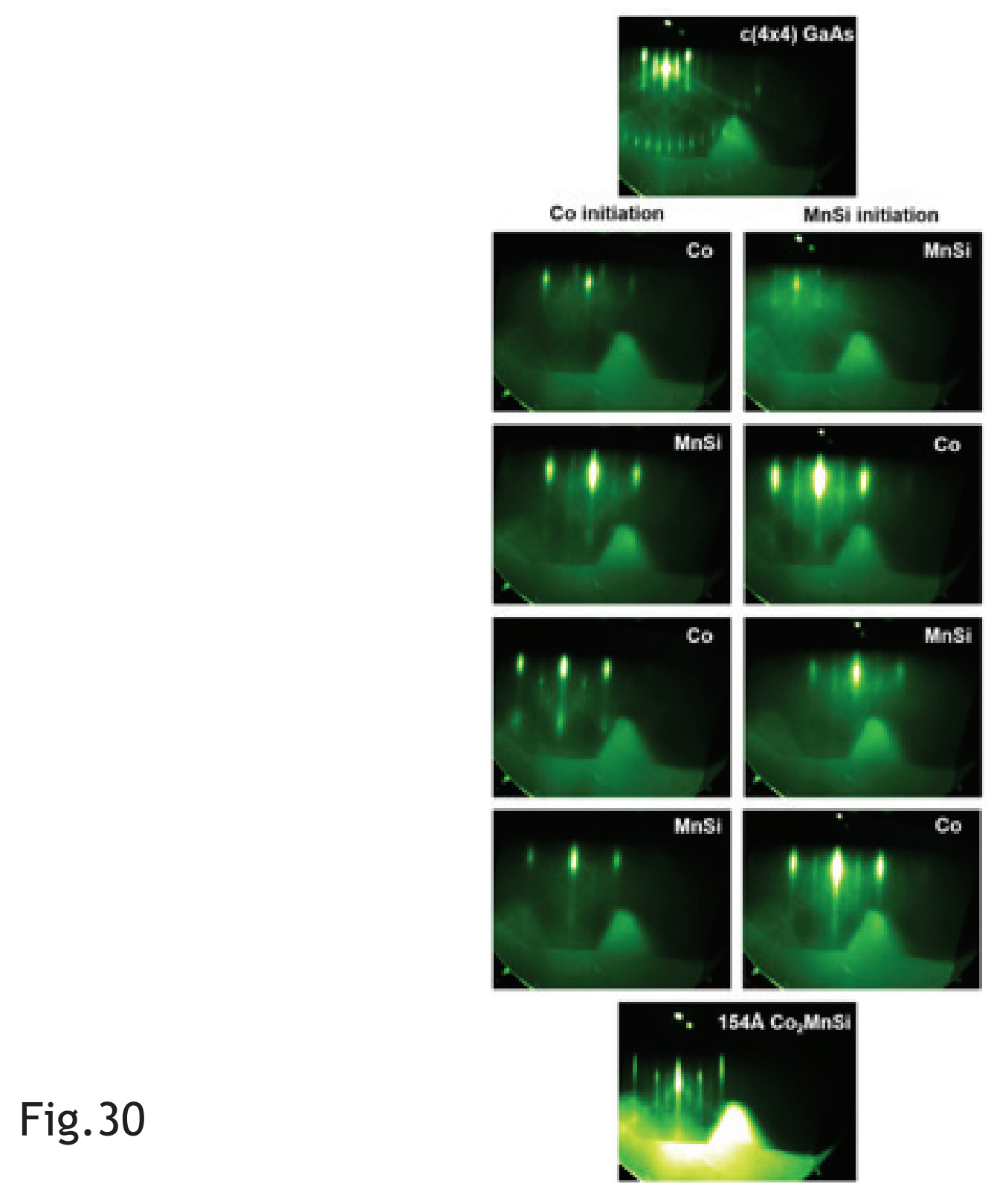



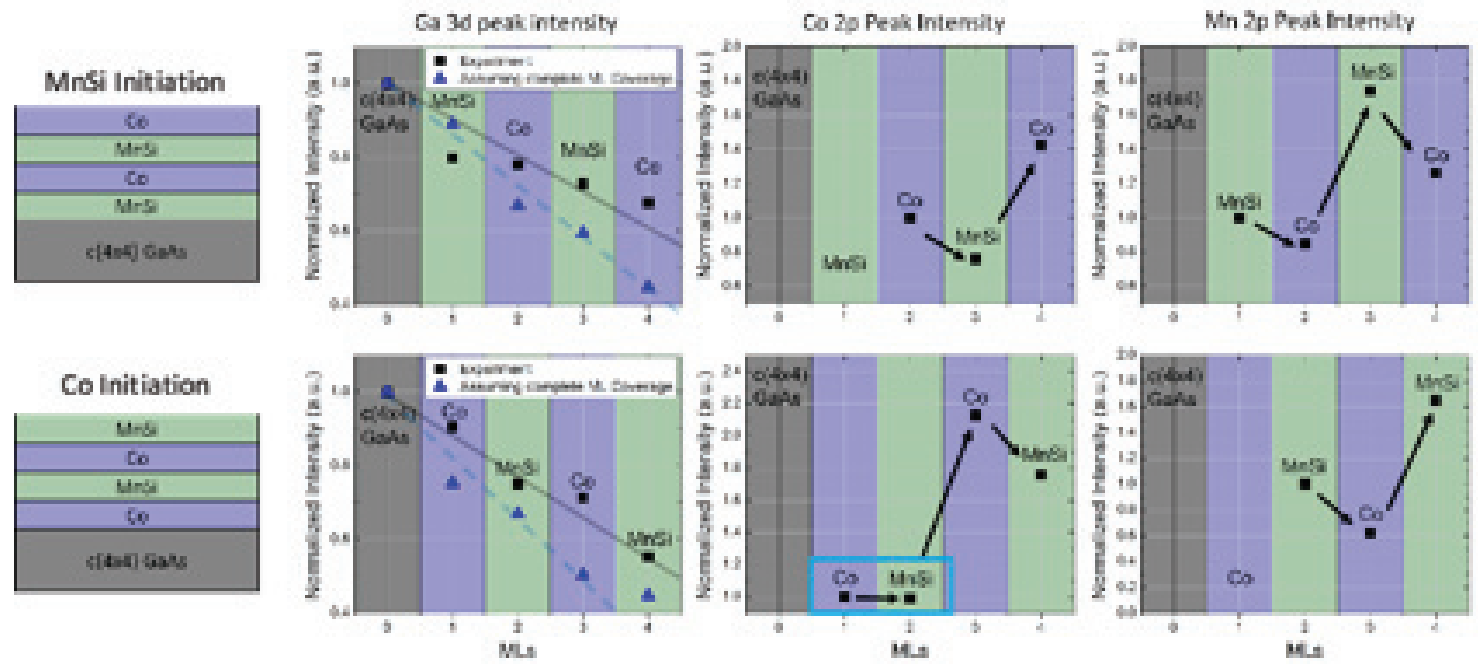

Fig. 31 


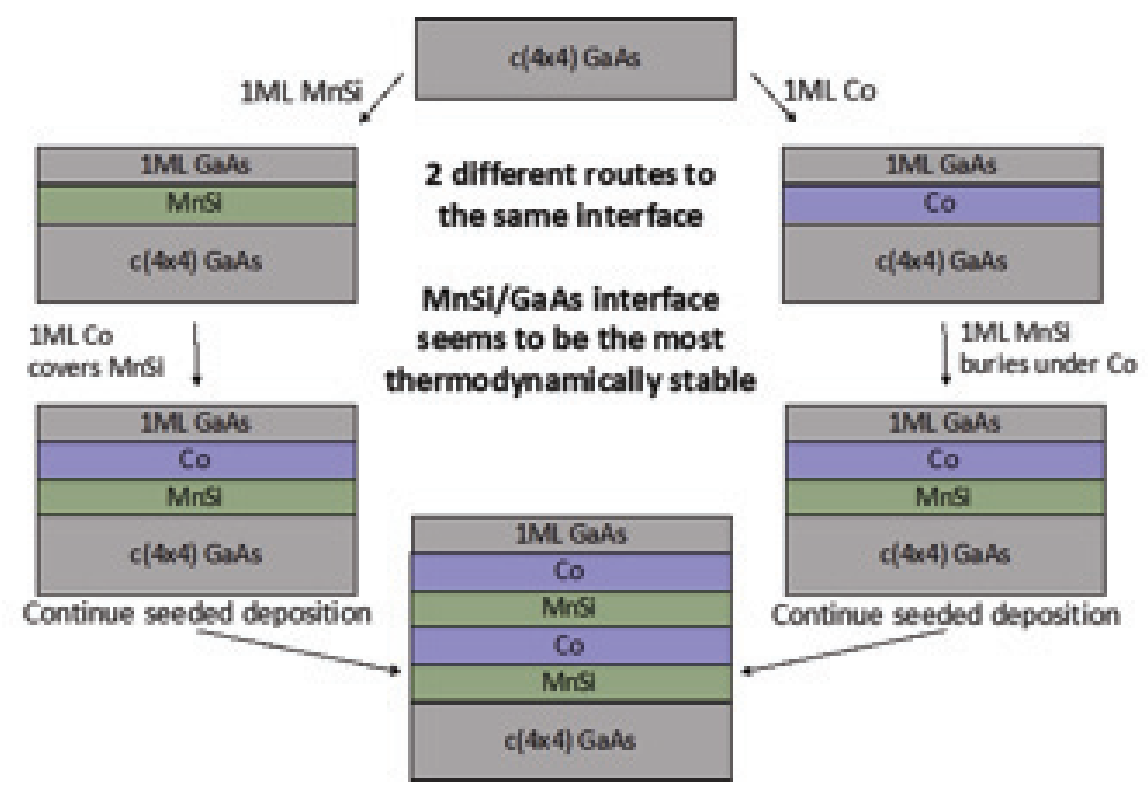

Fig. 32 


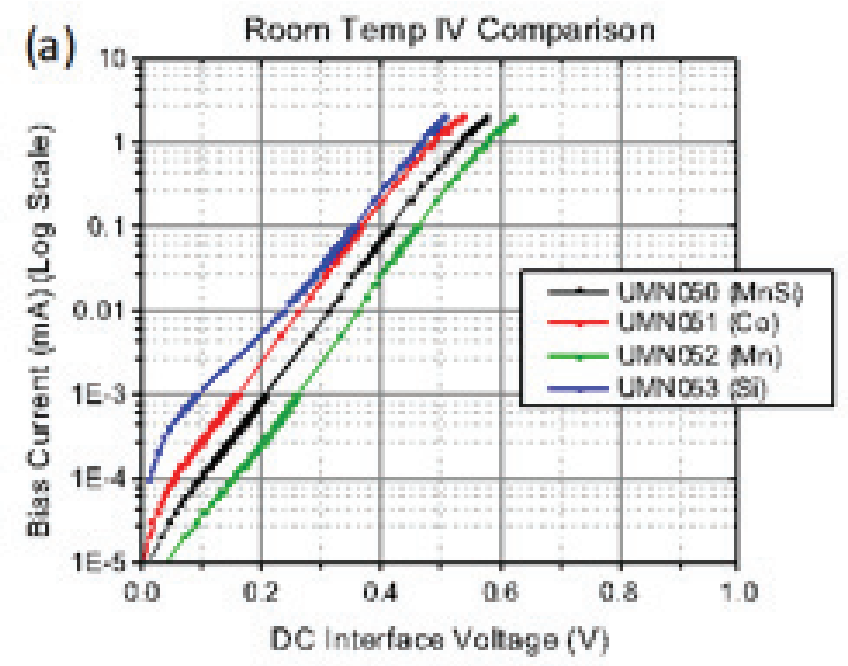

Fig. 33
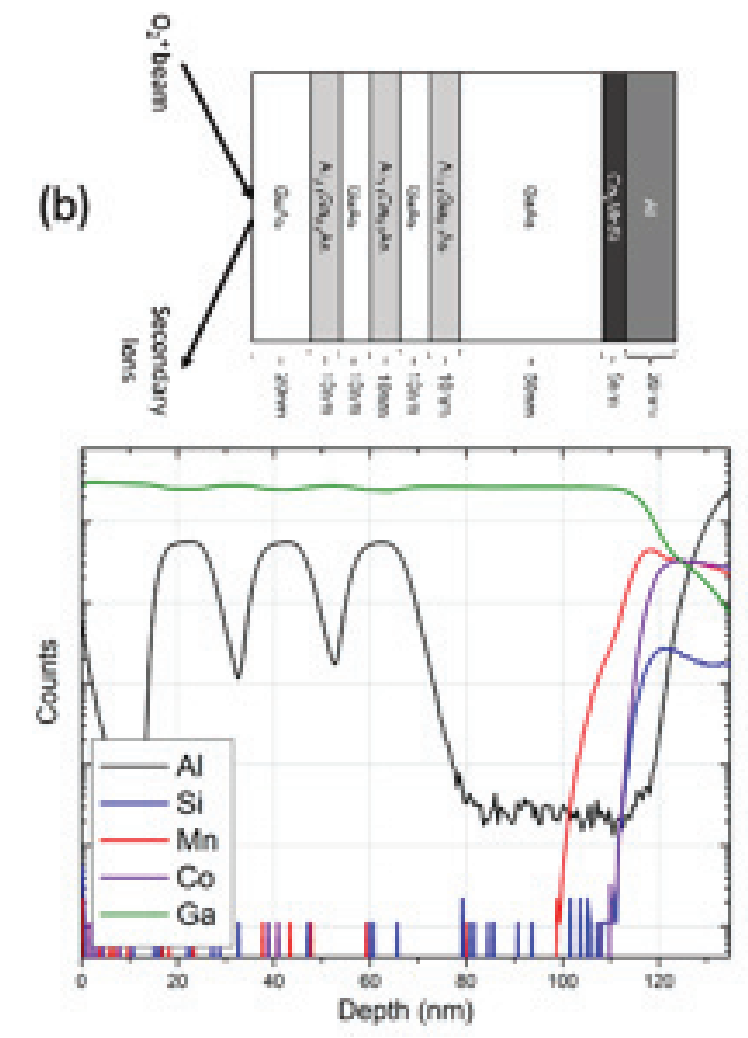


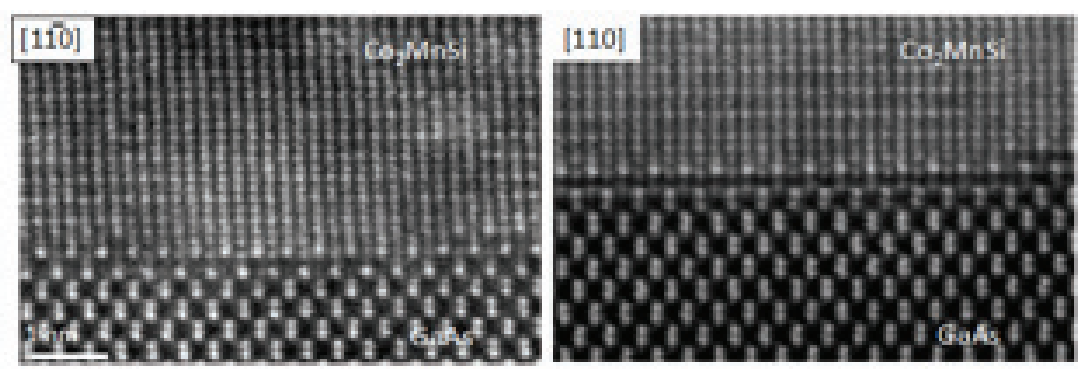

Fig. 34 

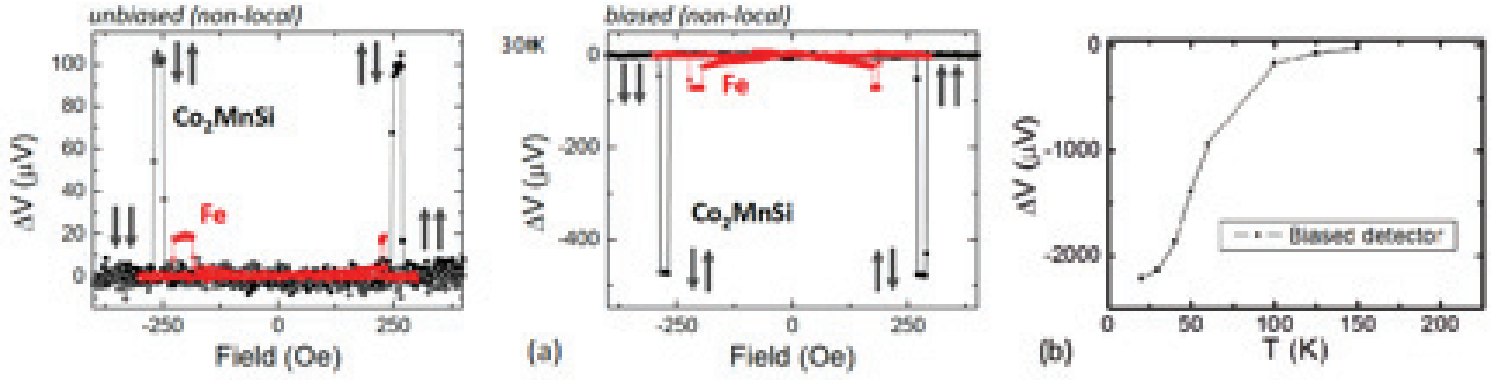

Fig. 35 

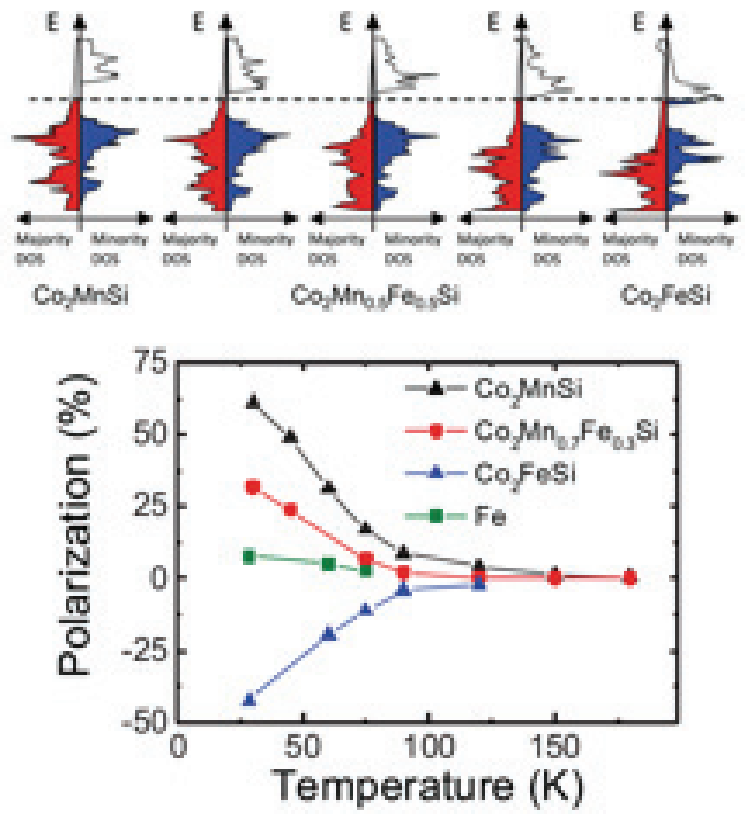

Fig. 36 


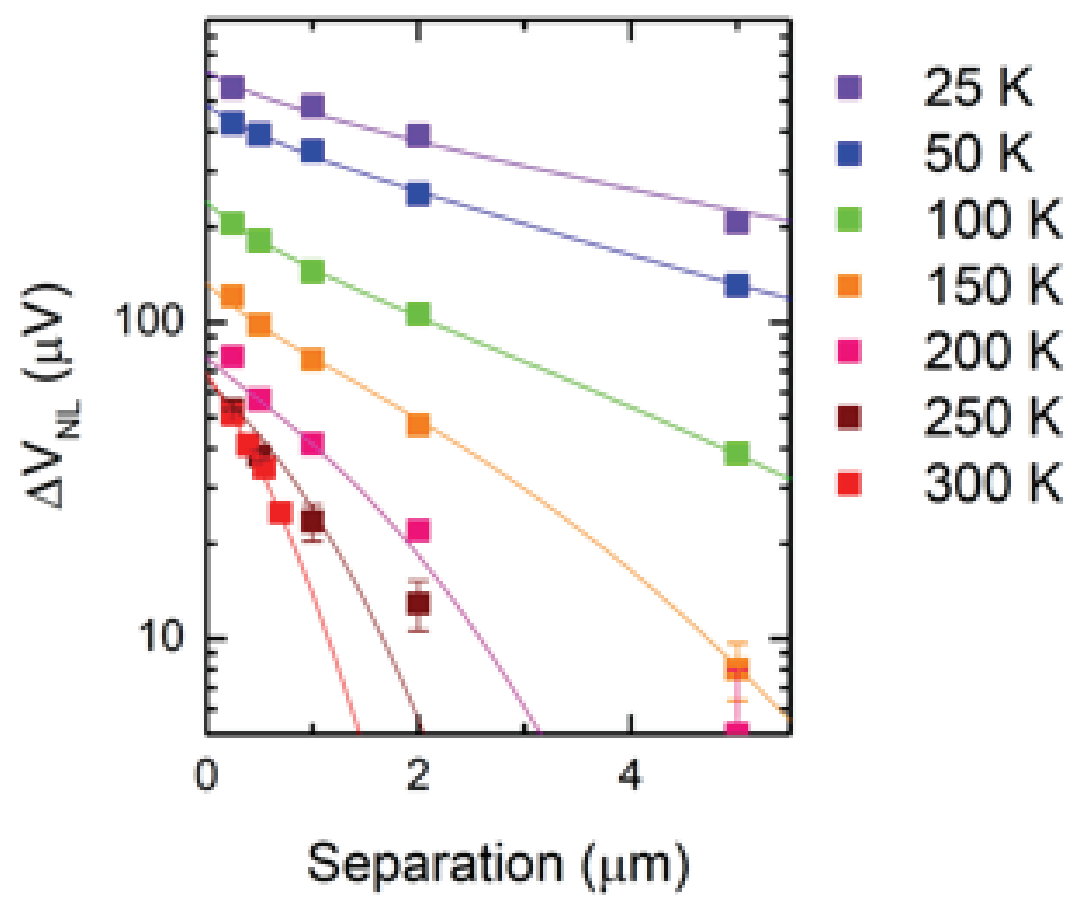

Fig. 37 